\title{
Some notes on the Feigin-Losev-Shoikhet integral conjecture
}

\author{
Ajay C. Ramadoss
}

\begin{abstract}
Given a holomorphic vector bundle $\mathcal{E}$ on a smooth connected compact complex manifold $X$, Feigin, Losev and Shoikhet [FLS] use a notion of completed Hochschild homology $\widehat{\mathrm{HH}}$ of $\operatorname{Diff}(\mathcal{E})$ such that $\widehat{\mathrm{HH}}_{0}(\operatorname{Diff}(\mathcal{E}))$ is isomorphic to $\mathrm{H}^{2 n}(X, \mathbb{C})$. On the other hand, they construct a linear functional on $\widehat{\mathrm{HH}}_{0}(\operatorname{Diff}(\mathcal{E}))$. This therefore gives rise to a linear functional $I_{\mathscr{E}}$ on $\mathrm{H}^{2 n}(X, \mathbb{C})$. They show that this functional is $\int_{X}$ if $\mathcal{E}$ has non-zero Euler characteristic. They conjecture that this functional is $\int_{X}$ for all $\mathcal{E}$.

In this article it is proved that $I_{\mathcal{E}}=I_{\mathscr{F}}$ for any pair $(\mathcal{E}, \mathcal{F})$ of holomorphic vector bundles on $X$. In particular, if $X$ has one vector bundle with non-zero Euler characteristic, then $I_{\mathscr{E}}=\int_{X}$ for every vector bundle $\mathcal{E}$ on $X$.

In [FLS] there is also used a notion of completed cyclic homology $\widehat{\mathrm{HC}}$ of $\mathscr{D i f f}(\mathcal{E})$ such that $\widehat{\mathrm{HC}}_{-i}(\operatorname{Diff}(\mathcal{E})) \simeq \mathrm{H}^{2 n-i}(X, \mathbb{C}) \oplus \mathrm{H}^{2 n-i+2}(X, \mathbb{C}) \oplus \cdots$. The construction yielding $I_{\mathcal{E}}$ generalizes to give linear functionals on $\widehat{\mathrm{HC}}-2 i(\mathscr{D i f f}(\mathcal{E}))$ for each $i \geq 0$. The linear functional

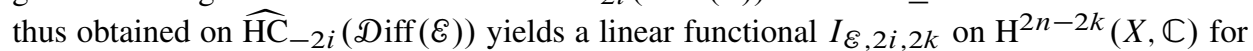
$0 \leq k \leq i$. It is conjectured in [FLS] that $I_{\mathcal{E}, 2,0}=\int_{X}$, and a further conjecture about $I_{\mathcal{E}, 2,2}$ is made.

In this article we prove that $I_{\mathcal{E}, 2 i, 0}=I_{\mathscr{E}}$ for all $i \geq 0$. In particular, if $X$ has at least one vector bundle with non-zero Euler characteristic, then $I_{\mathcal{E}, 2 i, 0}=\int_{X}$. We also prove that $I_{\mathcal{E}, 2 i, 2 k}=0$ for $k>0$. The latter is stronger than what is expected in [FLS] when $i=k=1$.
\end{abstract}

Mathematics Subject Classification (2000). 16E40.

Keywords. Hochschild cocycle, $A_{\infty}$-morphism, differential operators, Dolbeaux complex, FLS functional, supertrace, completed Hochschild homology, completed cyclic homology, integral operator, kernel.

\section{Introduction}

Let $X$ be a smooth connected compact complex manifold such that $\operatorname{dim}_{\mathbb{C}} X=n$. Let $\mathcal{E}$ be a holomorphic vector bundle on $X$. Let $\mathscr{D i f f}(\mathcal{E})$ be the sheaf of holomorphic differential operators on $\mathcal{E}$. Let $\operatorname{Diff}(\mathcal{E})$ be the algebra of global sections of $\mathscr{D i f f}(\mathcal{E})$. One is interested in understanding the Hochschild homology of $\operatorname{Diff}(\mathcal{E})$. This is, however, not an object that one can easily analyze. 
Let $\operatorname{Dolb}^{\bullet}\left(X, \mathcal{O}_{X}\right)$ denote the Dolbeaux resolution of the sheaf $\mathcal{O}_{X}$ of holomorphic functions on $X$. Let $\operatorname{Diff}^{\bullet}(\mathcal{E})=\operatorname{Dolb}^{\bullet}\left(X, \mathcal{O}_{X}\right) \otimes_{\mathcal{O}_{X}} \operatorname{Diff}(\mathcal{E})$. Following [FLS] we replace $\operatorname{Diff}(\mathcal{E})$ by

$$
\operatorname{Diff}^{\bullet}(\mathcal{E}):=\Gamma\left(X, \operatorname{Diff}^{\bullet}(\mathcal{E})\right)
$$

Let $K_{\mathcal{E}}^{\bullet}$ denote the complex $\Gamma_{C} \infty\left(X, \operatorname{Dolb}^{\bullet}\left(X, \mathcal{O}_{X}\right) \otimes_{\mathcal{O}_{X}} \mathcal{E}\right)$ of smooth global sections of $\operatorname{Dolb}^{\bullet}\left(X, \mathcal{O}_{X}\right) \otimes_{\mathcal{O}_{X}} \mathcal{E}$. This is the Dolbeaux complex of $\mathcal{E}$. If $\mathcal{D}$ is a holomorphic differential operator on $\mathcal{E}$, then $\mathscr{D}$ gives rise to a $\mathbb{C}$-endomorphism of the complex $K_{\mathscr{E}}^{\bullet}$. It follows that $\mathscr{D}$ induces an endomorphism $\mathscr{D}_{*}: \mathrm{H}^{i}(X, \mathcal{E}) \rightarrow$ $\mathrm{H}^{i}(X, \mathcal{E})$ for each $i$. The supertrace of $\mathcal{D}$ is given by

$$
\operatorname{str}(\mathcal{D}):=\sum_{i}(-1)^{i} \operatorname{tr}_{\mathrm{H}^{i}(X, \mathcal{E})} \mathcal{D}_{*} .
$$

Note that $\operatorname{Diff}(\mathcal{E})$ is a subalgebra of $\operatorname{Diff}^{\bullet}(\mathcal{E})$. It follows that there is a natural map from the $i$-th Hochschild homology of $\operatorname{Diff}(\mathcal{E})$ to that of $\operatorname{Diff}^{\bullet}(\mathcal{E})$ for all $i$. Also recall that if $\mathcal{A}$ is any associative $\mathbb{C}$-algebra concentrated in degree 0 then the 0 -th Hochschild homology $\mathrm{HH}_{0}(\mathcal{A})$ of $\mathcal{A}$ is the Abelianization of $\mathcal{A}$. It follows that any trace on $\mathcal{A}$ is a linear functional on $\mathrm{HH}_{0}(\mathcal{A})$ and a Hochschild 0-cocycle of $A$. Note that the supertrace described in the previous paragraph is a Hochschild 0-cocycle of $\operatorname{Diff}(\varepsilon)$.

In [FLS], a Hochschild 0-cocycle tr on $\operatorname{Diff}^{\bullet}(\mathcal{E})$ is constructed algebraically. This Hochschild cocycle is constructed in such a way that the following diagram commutes:

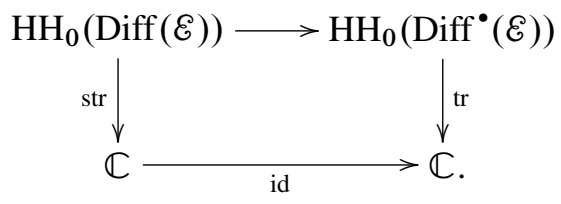

The top row of the above diagram is the natural map from $\mathrm{HH}_{0}(\operatorname{Diff}(\varepsilon))$ to $\mathrm{HH}_{0}\left(\right.$ Diff $\left.^{\bullet}(\mathcal{E})\right)$ arising out of the natural inclusion of Diff $(\mathcal{E})$ as a subalgebra of $\operatorname{Diff}^{\bullet}(\varepsilon)$.

The theory that is more related to the cohomology of $X$ is, however, the completed Hochschild homology of the sheaf $\mathscr{D i f f}(\mathcal{E})$ of holomorphic differential operators on $\mathcal{E}$. It is defined in Section 3. Let $\widehat{\mathrm{HH}}_{i}(\mathscr{D i f f}(\mathcal{E}))$ denote the $i$-th completed Hochschild homology of $\mathscr{D i f f}(\mathcal{E})$. By Lemma 3 we have the following generalization of a theorem of Brylinski [Bryl]:

$$
\widehat{\mathrm{HH}}_{-i}(\operatorname{Diff}(\mathcal{E})) \simeq \mathrm{H}^{2 n-i}(X, \mathbb{C}) .
$$

Call this isomorphism $\beta_{\mathcal{E}}$. This is the key property of $\widehat{\mathrm{HH}}_{*}(\mathcal{D i f f}(\mathcal{E}))$ that relates it to the cohomology of $X$ with coefficients in $\mathbb{C}$. In [FLS] there is also a notion of completed Hochschild homology that has the same relation with $\mathrm{H}^{*}(X, \mathbb{C})$. However, as pointed out in [FLS], understanding the isomorphism $\beta_{\mathcal{E}}$ is easier with the definition of completed Hochschild homology used here. 
In [FLS] it is shown that the Hochschild cocycle tr of Diff ${ }^{\bullet}(\mathcal{E})$ "extends" to yield a linear functional tr on $\widehat{\mathrm{HH}}_{0}(\mathscr{D i f f}(\mathcal{E}))$. In this article, we are forced to redo this very step in Section 3 since we use a definition of $\widehat{\mathrm{HH}}_{*}(\mathscr{D i f f}(\mathcal{E}))$ that is a priori different from that in [FLS]. Our definition of $\widehat{\mathrm{HH}}_{*}(\mathscr{D i f f}(\mathscr{E}))$ implies that there is a natural map from $\mathrm{HH}_{0}(\operatorname{Diff}(\mathcal{E}))$ to $\widehat{\mathrm{HH}}_{0}(\operatorname{Diff}(\mathcal{E}))$ (see Section 3 for more details). The outcome of our "extending" tr to a linear functional tr on $\widehat{\mathrm{HH}}_{0}(\mathscr{D i f f}(\mathscr{E}))$ is the following commutative diagram:

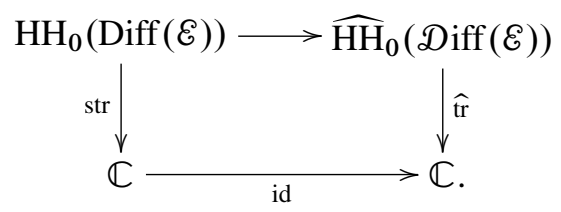

The linear functional we construct on $\widehat{\mathrm{HH}}_{0}(\mathscr{D i f f}(\mathcal{E}))$ still uses the basic construction of [FLS] and, as will be clear later in this article, yields an algebraic construction of $\int_{X}$ when $X$ has at least one vector bundle with non-zero Euler characteristic.

Denote the composite map $\widehat{\operatorname{tr}} \circ \beta_{\mathcal{E}}^{-1}: \mathrm{H}^{2 n}(X, \mathbb{C}) \rightarrow \mathbb{C}$ by $I_{\mathcal{E}} . I_{\mathcal{E}}$ is a linear functional on $\mathrm{H}^{2 n}(X, \mathbb{C})$.

The following theorem was proven in [FLS]. The proof goes through in our setup as well.

Theorem 1 ([FLS]). If the Euler characteristic $\chi_{\mathcal{E}}$ of $\mathcal{E}$ is non-zero, then $I_{\mathcal{E}}=\int_{X}$.

Further, in [FLS], it was conjectured that $I_{\mathcal{E}}=\int_{X}$ for all vector bundles $\mathcal{E}$, even those of zero Euler characteristic. Here we prove the following theorem.

Theorem 2. If $\mathcal{E}$ and $\mathcal{E}$ are two vector bundles on $X$, then $I_{\mathcal{E}}=I \mathscr{g}$.

The following corollary is immediate from this and Theorem 1.

Corollary 1. If $X$ has a vector bundle $\mathcal{E}$ whose Euler characteristic $\chi_{\mathcal{E}}$ is non-zero, then $I_{\mathcal{E}}=\int_{X}$ for any vector bundle $\mathcal{E}$ on $X$.

In particular, if $X$ is a smooth separated scheme over $\mathbb{C}$, then the skyscraper sheaf supported at a point $p$ of $X$ has non-zero Euler characteristic. On the other hand, it is a coherent $\mathcal{O}_{X}$-module. It therefore has a finite resolution by (holomorphic) vector bundles on $X$. It follows that at least one vector bundle in such a resolution has nonzero Euler characteristic. Thus, if $\mathcal{E}$ is a vector bundle on a smooth compact complex manifold $X$ that is a smooth separated scheme over $\mathbb{C}$, then $I_{\mathcal{E}}=\int_{X}$.

Note that if $\mathscr{D}$ is a global holomorphic differential operator on a vector bundle $\mathscr{E}$ on $X$, then $\mathscr{D}$ is a 0 -cycle of the Hochschild chain complex of Diff $(\mathscr{E})$. It follows that 
$\mathscr{D}$ yields an element in $\widehat{\mathrm{HH}}_{0}(\mathscr{D i f f}(\mathcal{E}))$ and hence an element in $\mathrm{H}^{2 n}(X, \mathbb{C})$, which will be denoted by [D]. It follows from Corollary 1 that if $X$ admits at least one vector bundle with non-zero Euler characteristic, then

$$
\operatorname{str}(\mathscr{D})=\int_{X}[\mathscr{D}] .
$$

The above statement is somewhat similar to a result (Corollary 5.6) in an article by P. Schapira and J.-P. Schneiders [S-S]. More recently, an entirely different approach has yielded a proof of a stronger version of this statement (the supertrace theorem) without the condition that $X$ admits a vector bundle with non-zero Euler characteristic [EnFe]. In a sequel to this article, we shall develop our approach further to show that $I_{\mathcal{E}}=\int_{X}$ for any vector bundle $\mathcal{E}$ on any compact complex manifold $X$. This yields another proof of the supertrace theorem different from the one in [EnFe].

One also defines the completed cyclic homology $\widehat{\mathrm{HC}}_{*}(\mathcal{D i f f}(\mathcal{E}))$ of $\mathscr{D i f f}(\mathcal{E})$. Further, as in [FLS], the construction of the linear functional on the 0-th completed Hochschild homology of $\operatorname{Diff}(\mathcal{E})$ is extended to the construction of multiple linear functionals $\widehat{t r}_{2 i}: \widehat{\mathrm{HC}}_{-2 i}(\mathscr{D i f f}(\mathcal{E})) \rightarrow \mathbb{C}$. On the other hand, it is shown that one has an isomorphism

$$
\widehat{\mathrm{HC}}_{-2 i}(\mathscr{D i f f}(\mathcal{E})) \simeq \mathrm{H}^{2 n}(X, \mathbb{C}) \oplus \mathrm{H}^{2 n-2}(X, \mathbb{C}) \oplus \cdots \oplus \mathrm{H}^{2 n-2 i}(X, \mathbb{C}) .
$$

Denote the inverse of this isomorphism by $I_{\mathcal{E}}^{i}$. Let $I_{\mathcal{E}, 2 i}=\widehat{\operatorname{tr}}_{2 i} \circ I_{\mathcal{E}}^{i}$. Then

$$
I_{\mathcal{E}, 2 i}: \mathrm{H}^{2 n}(X, \mathbb{C}) \oplus \mathrm{H}^{2 n-2}(X, \mathbb{C}) \oplus \cdots \oplus \mathrm{H}^{2 n-2 i}(X, \mathbb{C}) \rightarrow \mathbb{C} .
$$

For $0 \leq k \leq i$ let $I_{\mathcal{E}, 2 i, 2 k}: \mathrm{H}^{2 n-2 k}(X, \mathbb{C}) \rightarrow \mathbb{C}$ be the composition of $I_{\mathcal{E}, 2 i}$ with the inclusion of $\mathrm{H}^{2 n-2 k}(X, \mathbb{C})$ in $\mathrm{H}^{2 n}(X, \mathbb{C}) \oplus \mathrm{H}^{2 n-2}(X, \mathbb{C}) \oplus \cdots \oplus \mathrm{H}^{2 n-2 i}(X, \mathbb{C})$ as a direct summand. In [FLS] it is conjectured that $I_{\mathcal{E}, 2,0}=C \cdot \int_{X}$ where $C$ is a constant independent of $\mathcal{E}$. Furthermore, there is a prediction in [FLS] that $I_{\mathcal{E}, 2,2}$ is the integral over a cycle related to the Chern classes of $\mathcal{E}$ and those of vector bundles intrinsic to $X$. Here we prove the following theorem.

Theorem 3. $I_{\mathcal{E}, 2 i, 0}=I_{\mathcal{E}}$. Moreover, $I_{\mathcal{E}, 2 i, 2 k}=0$ if $k>0$.

The latter part is more than what is expected in [FLS], though a piece of circumstantial evidence for $I_{\mathcal{E}, 2,2}$ to be 0 is provided there. By this theorem and Corollary 1 , $I_{\mathcal{E}, 2 i, 0}=\int_{X}$ whenever $X$ has a vector bundle with non-zero Euler characteristic. In particular, this happens when $X$ is a smooth separated scheme over $\mathbb{C}$.

1.1. Outline of the article. The proofs of Theorems 2 and 3 are simple and involve two easy "bookkeeping lemmas" (Lemma 1 and Lemma 4). This is done in Sections 3 and 4 , respectively. 
Section 2 recalls basic notions about Hochschild homology that we require. In addition, Section 2 recalls the definition of an $A_{\infty}$-morphism between two differential graded algebras. It shows that an $A_{\infty}$-morphism $\mathcal{F}$ between two dg algebras $\mathcal{A}$ and $\mathscr{B}$ induces a map $\widetilde{F}_{\text {Hoch }}$ of complexes from the complex of Hochschild chains of $\mathcal{A}$ to that of $\mathscr{B}$ (Proposition 2). None of the material presented in this section is new. Proposition 2 is from [FLS].

The first part of Section 3 recalls the basic set up for this note from [FLS]. In particular, we recall the construction of the Hochschild 0-cocycle tr of Diff ${ }^{\bullet}(\mathscr{E})$ from [FLS]. The completed Hochschild homology $\widehat{\mathrm{HH}}_{*}(\operatorname{Diff}(\mathscr{E}))$ is defined in Section 3. We show that the formula for the Hochschild cocycle tr "extends" to a formula for a linear functional tr on $\widehat{\mathrm{HH}}_{0}(\operatorname{Diff}(\mathcal{E}))$. In [FLS] something very similar is done. The definition of $\widehat{\mathrm{HH}}_{*}(\mathscr{D i f f}(\mathscr{E}))$ that is used in this article is however a priori somewhat different from that used in [FLS]. This forces us to redo the parts where in [FLS] the Hochschild cocycle tr of Diff ${ }^{\bullet}(\mathcal{E})$ is extended to a linear functional on $\widehat{\mathrm{HH}}_{0}(\operatorname{Diff}(\mathcal{E}))$.

The last part of Section 3 is devoted to the "bookkeeping lemma for tr." (Lemma 1).

Section 4 is devoted to understanding the relation between $\widehat{\mathrm{HH}}_{*}(\mathscr{D i f f}(\mathscr{E}))$ and $\mathrm{H}^{*}(X, \mathbb{C})$. In particular, we prove that

$$
\widehat{\mathrm{HH}}_{-i}(\operatorname{Diff}(\mathcal{E})) \simeq \mathrm{H}^{2 n-i}(X, \mathbb{C})
$$

(Corollary 6). For $\mathcal{E}=\mathcal{O}_{X}$, this was proven by Brylinski [Bryl]. Let $\beta_{\mathcal{E}}$ denote the isomorphism between $\widehat{\mathrm{HH}}_{-i}(\operatorname{Diff}(\mathcal{E}))$ and $\mathrm{H}^{2 n-i}(X, \mathbb{C})$. Section 4 also proves the "bookkeeping lemma for $\beta$ " (Lemma 4). Theorem 2 is an immediate consequence of Lemma 1 and Lemma 4.

Section 5 has four subsections. The first subsection recalls the basic notions of cyclic homology that we require. In the second subsection we carefully examine the linear functionals on the completed cyclic homology of $\mathscr{D i f f}(\mathcal{E})$. The third subsection analyses the relation between the completed cyclic homology of $\operatorname{Diff}(\mathscr{E})$ and $\mathrm{H}^{*}(X, \mathbb{C})$. In the final subsection we write down the final steps of the proof of Theorem 3 .

Note. All complexes that appear in this article are cochain complexes by convention. The term Hochschild cocycle of a dg algebra shall only refer to cocycles of the complex of Hochschild cochains of that algebra as defined in Definition 2.

Acknowledgements. This article and its sequel would not have been what they are but for the help I received from many quarters. I am very grateful to Prof. Boris Tsygan for going through the article carefully, introducing me to a article of N. Teleman [Tel] and for some very useful comments and suggestions. I am also very grateful to Prof. Madhav Nori for some very useful discussions, comments and suggestions. Heartfelt thanks are also due to Prof. Ryszard Nest and Prof. Alexander Gorokhovsky for helping me understand the correct completed tensor product to be used. I am also grateful to Prof. Shrawan Kumar and Dr. Victor Protsak for useful discussions and 
to the referee for going through this article carefully and helping me streamline its presentation.

\section{Basic results about Hochschild homology}

Conventions used in this section. The term "dg algebra" in this section refers to a differential graded $\mathbb{C}$-algebra with unit.

All complexes of $\mathbb{C}$-vector space are cochain complexes, i.e, the differential of any complex of $\mathbb{C}$-vector spaces has degree +1 .

\subsection{The Hochschild chain complex and bar complex of a dg algebra}

Definition 1. If $\mathcal{A}$ is a differential graded $\mathbb{C}$-algebra with differential $\delta$, the complex of Hochschild chains $C^{\bullet}(\mathcal{A})$ is the cochain complex obtained by equipping the graded $\mathbb{C}$-vector space $\bigoplus_{i \geq 1} \mathcal{A}[1]^{\otimes i}[-1]$ with the Hochschild differential. The Hochschild differential $d$ is given by the formula

$$
\begin{aligned}
d\left(a_{0} \otimes \cdots \otimes a_{n}\right)= & \sum_{i=0}^{i=n-1}(-1)^{\left(d_{0}+\cdots+d_{i}+i+1\right)} a_{0} \otimes \cdots \otimes a_{i} a_{i+1} \otimes \cdots \otimes a_{n} \\
& +(-1)^{\left(d_{n}+1\right)\left(d_{0}+\cdots+d_{n-1}+n-1\right)} a_{n} a_{0} \otimes a_{1} \otimes \cdots \otimes a_{n-1} \\
& +\sum_{j=0}^{j=n}(-1)^{\left(d_{0}+\cdots+d_{j-1}+j\right)} a_{0} \otimes \cdots \otimes \delta\left(a_{j}\right) \otimes \cdots \otimes a_{n}
\end{aligned}
$$

for homogenous elements $a_{0}, \ldots, a_{n}$ of $\mathcal{A}$ of degrees $d_{0}, \ldots, d_{n}$, respectively.

Let $\tau_{n}: \mathcal{A}^{\otimes n} \rightarrow \mathcal{A}^{\otimes n}$ denote the endomorphism given by the formula

$$
a_{1} \otimes \cdots \otimes a_{n} \leadsto(-1)^{\left(d_{n}+1\right)\left(d_{1}+\cdots+d_{n-1}+n-1\right)} a_{n} \otimes a_{1} \otimes \cdots \otimes a_{n-1}
$$

for homogenous elements $a_{1}, \ldots, a_{n}$ of $\mathcal{A}$ of degrees $d_{1}, \ldots, d_{n}$, respectively. Let $\partial_{1}: \mathcal{A}^{\otimes n} \rightarrow \mathcal{A}^{\otimes n-1}$ denote the morphism given by the formula

$$
a_{1} \otimes \cdots \otimes a_{n} \leadsto(-1)^{d_{1}+1} a_{1} a_{2} \otimes \cdots \otimes a_{n}
$$

for homogenous elements $a_{1}, \ldots, a_{n}$ of $\mathcal{A}$ of degrees $d_{1}, \ldots, d_{n}$, respectively. Then the Hochschild differential on $\mathcal{A}^{\otimes k}$ is also given by the formula

$$
\sum_{i=1}^{i=k} \tau_{k-1}^{i-1} \circ \partial_{1} \circ \tau_{k}^{k-i+1}+\sum_{i=1}^{i=k} \tau_{k}^{i-1} \circ(\delta \otimes \mathrm{id} \otimes \cdots \otimes \mathrm{id}) \circ \tau_{k}^{k-i+1} .
$$


Contrary to the standard practice, we will refer to a degree $-n$ cocycle of $C^{\bullet}(\mathcal{A})$ as a Hochschild $n$-cycle of $\mathcal{A}$.

The Hochschild homology $\mathrm{HH}_{-n}(\mathcal{A})$ of $\mathcal{A}$ is the $-n$-th cohomology of the complex $C^{\bullet}(\mathcal{A})$. By the definition of the Hochschild complex, it is clear that if $\mathcal{A}$ is a $\mathbb{C}$-algebra (i.e., $\mathcal{A}$ is concentrated in degree 0 ), then $\mathrm{HH}_{0}(\mathcal{A})=\frac{\mathcal{A}}{[\mathscr{A}, \mathcal{A}]}$ where $[\mathcal{A}, \mathcal{A}]$ is the commutator of $\mathcal{A}$. Also, if $\mathscr{A}$ is a $\mathbb{C}$-algebra, then $\mathrm{HH}_{-n}(\mathscr{A})=0$ if $n<0$. And, if $\mathcal{A}$ is dg algebra, then elements of $\operatorname{ker}(\delta) \subset \mathcal{A}^{0}$ are 0 -Hochschild cycles of $C^{\bullet}(\mathcal{A})$ where $\delta$ denotes the internal differential on $\mathcal{A}$. It follows that there is a canonical map $\operatorname{ker}(\delta) \rightarrow \mathrm{HH}_{0}(\mathcal{A})$.

Definition 2. The complex of Hochschild cochains of $\mathcal{A}$ is the complex whose $-i$-th term is $\operatorname{Hom}_{\mathbb{C}}\left(C^{i}(\mathcal{A}), \mathbb{C}\right)$ with differential induced by that on $C^{\bullet}(\mathcal{A})$. A Hochschild cocycle of $\mathcal{A}$ is a cocycle of the complex of Hochschild cochains of $\mathcal{A}$. Note that a Hochschild 0-cocycle of $\mathcal{A}$ induces a $\mathbb{C}$-linear functional on $\mathrm{HH}_{0}(\mathcal{A})$.

Definition 3. The bar complex $\operatorname{bar}^{\bullet}(\mathcal{A})$ of $\mathcal{A}$ is the cochain complex obtained by equipping the graded $\mathbb{C}$-vector space $\bigoplus_{i \geq 1} \mathcal{A}[1]^{\otimes i}[-1]$ with the bar differential. The bar differential is given by the formula

$$
\begin{aligned}
d\left(a_{0} \otimes \cdots \otimes a_{n}\right)= & \sum_{i=0}^{i=n-1}(-1)^{\left(d_{0}+\cdots+d_{i}+i+1\right)} a_{0} \otimes \cdots \otimes a_{i} a_{i+1} \otimes \cdots \otimes a_{n} \\
& +\sum_{j=0}^{j=n}(-1)^{\left(d_{0}+\cdots+d_{j-1}+j\right)} a_{0} \otimes \cdots \otimes \delta\left(a_{j}\right) \otimes \cdots \otimes a_{n}
\end{aligned}
$$

for homogenous elements $a_{0}, \ldots, a_{n}$ of $\mathcal{A}$ of degrees $d_{0}, \ldots, d_{n}$, respectively.

The bar differential on $\mathcal{A}^{\otimes k}$ is also given by the formula

$$
\sum_{i=1}^{i=k-1} \tau_{k-1}^{i-1} \circ \partial_{1} \circ \tau_{k}^{k-i+1}+\sum_{i=1}^{i=k} \tau_{k}^{i-1} \circ(\delta \otimes \mathrm{id} \otimes \cdots \otimes \mathrm{id}) \circ \tau_{k}^{k-i+1} .
$$

We recall that if $s_{n}: \operatorname{bar}^{\bullet}(\mathcal{A}) \rightarrow \operatorname{bar}^{\bullet-1}(\mathcal{A})$ is the map $a_{0} \otimes \cdots \otimes a_{n} \leadsto-1 \otimes$ $a_{0} \otimes \cdots \otimes a_{n}$ then the maps $\left\{s_{n}\right\}, n \geq-1$, give a homotopy between id: $\operatorname{bar}^{\bullet}(\mathcal{A}) \rightarrow$ $\operatorname{bar}^{\bullet}(\mathcal{A})$ and $0: \operatorname{bar}^{\bullet}(\mathcal{A}) \rightarrow \operatorname{bar}^{\bullet}(\mathcal{A})$. It follows that $\operatorname{bar}^{\bullet}(\mathcal{A})$ is acyclic.

If $V^{\bullet}$ is a finite dimensional graded $\mathbb{C}$-vector space then $\operatorname{End}\left(V^{\bullet}\right)=$ $\bigoplus_{i, j} \operatorname{Hom}\left(V^{i}, V^{j}\right)$. Let $\pi_{i, j}$ denote the projection from $\operatorname{End}\left(V^{\bullet}\right)$ to $\operatorname{Hom}\left(V^{i}, V^{j}\right)$. If $M \in \operatorname{End}\left(V^{\bullet}\right)$, the supertrace of $M$ is the alternating sum $\sum_{i}(-1)^{i} \operatorname{tr}\left(\pi_{i, i}(M)\right)$. We recall the following proposition from [FLS].

Proposition 1. Let $V^{\bullet}$ be a finite dimensional graded $\mathbb{C}$-vector space with zero differential. The the following holds: 
(1) $\mathrm{HH}_{i}\left(\operatorname{End}\left(V^{\bullet}\right)\right)=0$ for $i \neq 0$.

(2) $\mathrm{HH}_{0}\left(\operatorname{End}\left(V^{\bullet}\right)\right) \simeq \mathbb{C}$.

(3) The composite $\operatorname{End}\left(V^{\bullet}\right)^{0} \rightarrow \mathrm{HH}_{0}\left(\operatorname{End}\left(V^{\bullet}\right)\right) \rightarrow \mathbb{C}$ takes an element of $\operatorname{End}\left(V^{\bullet}\right)^{0}$ to its supertrace.

(4) The isomorphism $\mathrm{HH}_{0}\left(\operatorname{End}\left(V^{\bullet}\right)\right) \simeq \mathbb{C}$ takes the class in $\mathrm{HH}_{0}\left(\operatorname{End}\left(V^{\bullet}\right)\right)$ of a Hochschild 0 -cycle in $\operatorname{End}\left(V^{\bullet}\right)^{\otimes k}$ to 0 for all $k \geq 2$.

Proof. The proof is a trivial modification of the proof of the Morita invariance of Hochschild homology for matrices in Loday [2] (see Theorem 1.2.4 of [2]).

We may therefore denote the isomorphism $\mathrm{HH}_{0}\left(\operatorname{End}\left(V^{\bullet}\right)\right) \simeq \mathbb{C}$ by str.

2.2. $A_{\infty}$-morphisms between dg algebras. Let $\mathcal{A}$ and $\mathcal{B}$ be two dg algebras. Let $\mathcal{F}_{i}: \mathcal{A}^{\otimes i} \rightarrow \mathscr{B}\left[n_{i}\right]$ be $\mathbb{C}$-linear maps with $n_{i} \in \mathbb{Z}$ for each $i$. If $k_{1}, \ldots, k_{l}$ are positive integers such that $\sum_{j} k_{j}=k$ and if $\sum_{j} n_{j}=n$, then $\mathcal{F}_{k_{1}} \otimes \cdots \otimes \mathcal{F}_{k_{l}}$ will denote the $\mathbb{C}$-linear map from $\mathcal{A}^{\otimes k}$ to $\mathscr{B}^{\otimes l}[n]$ such that

$$
\begin{aligned}
\mathscr{F}_{k_{1}} & \otimes \cdots \otimes \mathscr{F}_{k_{l}}\left(a_{1} \otimes \cdots \otimes a_{k}\right) \\
& =\widetilde{F}_{k_{1}}\left(a_{1} \otimes \cdots \otimes a_{k_{1}}\right) \otimes \cdots \otimes \mathscr{F}_{k_{l}}\left(a_{k_{1}+\cdots+k_{l-1}+1} \otimes \cdots \otimes a_{k}\right) .
\end{aligned}
$$

Definition 4. An $A_{\infty}$-morphism $\mathcal{F}$ from a $\mathrm{dg}$ algebra $\mathcal{A}$ to a dg algebra $\mathcal{B}$ is a collection of maps

$$
\widetilde{F}_{k}: \mathcal{A}^{\otimes k} \rightarrow \mathscr{B}[1-k]
$$

for all $k \geq 1$ such that the map $\mathcal{F}_{\text {bar }}: \operatorname{bar}^{\bullet}(\mathcal{A}) \rightarrow \operatorname{bar}^{\bullet}(\mathcal{B})$ defined by

$$
\mathcal{F}_{\text {bar }}\left(a_{1} \otimes \cdots \otimes a_{k}\right)=\sum_{\substack{\left(k_{1}, \ldots, k_{l}\right) \\ l>0, \sum_{j} k_{j}=k}} \mathcal{F}_{k_{1}} \otimes \cdots \otimes \mathcal{F}_{k_{l}}\left(a_{1} \otimes \cdots \otimes a_{k}\right)
$$

is a morphism of complexes from $\operatorname{bar}^{\bullet}(\mathcal{A})$ to $\operatorname{bar}^{\bullet}(\mathscr{B})$. The maps $\mathcal{F}_{k}$ are called the Taylor components of $\mathcal{F}$.

The condition that $\mathcal{F}_{\text {bar }}$ commutes with the differentials on the bar complexes of $\mathcal{A}$ and $\mathscr{B}$ respectively is equivalent to the condition that the maps $\widetilde{F}_{k}$ satisfy the following relations:

$$
\begin{aligned}
& \pm \mathscr{F}_{k-1}\left(a_{1} \cdot a_{2} \otimes a_{3} \otimes \cdots \otimes a_{k}\right) \mp \mathscr{F}_{k-1}\left(a_{1} \otimes a_{2} \cdot a_{3} \otimes \cdots \otimes a_{k}\right) \\
& \quad \pm \mathcal{F}_{k-1}\left(a_{1} \otimes a_{3} \otimes \cdots \otimes a_{k-1} \cdot a_{k}\right) \pm \mathscr{F}_{k}\left(\delta\left(a_{1} \otimes a_{2} \otimes \cdots \otimes a_{k}\right)\right) \\
& \quad \pm \delta\left(\mathcal{F}_{k}\left(a_{1} \otimes \cdots \otimes a_{k}\right)\right) \\
& \quad \pm \sum_{l=1}^{l=k-1} \pm \mathcal{F}_{l}\left(a_{1} \otimes \cdots \otimes a_{l}\right) \circ \mathscr{F}_{k-l}\left(a_{l+1} \otimes \cdots \otimes a_{k}\right)=0, \quad k \geq 1
\end{aligned}
$$

We recall the following proposition from [FLS]. 
Proposition 2. An $A_{\infty}$-morphism $\mathcal{F}$ from an associative dg algebra $\mathcal{A}$ to an associative dg algebra $\mathfrak{B}$ induces a map $\widetilde{F}_{\mathrm{Hoch}}$ of complexes from the $C^{\bullet}(\mathcal{A})$ to $C^{\bullet}(\mathcal{B})$.

We recall the proof of this proposition from [FLS].

Proof. Denote by $\tau: \mathcal{A}^{\otimes k} \rightarrow \mathcal{A}^{\otimes k}$ the map which takes $a_{1} \otimes \cdots \otimes a_{k}$ to $(-1)^{\left(d_{k}+1\right)\left(d_{1}+\cdots+d_{k-1}+k-1\right)} a_{k} \otimes a_{1} \otimes \cdots \otimes a_{k-1}$ for homogenous elements $a_{1}, \ldots, a_{k}$ of $\mathcal{A}$ of degrees $d_{1}, \ldots, d_{k}$, respectively. Consider the map $\mathcal{F}_{\text {Hoch }}: C^{\bullet}(\mathcal{A}) \rightarrow C^{\bullet}(\mathcal{B})$ defined by the formula

$$
\begin{aligned}
\mathscr{F}_{\text {Hoch }}\left(a_{1} \otimes \cdots \otimes a_{k}\right)= & \sum_{\substack{\left(k_{1}, \ldots, k_{l}\right), l>0, \sum_{j} k_{j}=k}}\left[\mathcal{F}_{k_{1}} \otimes \cdots \otimes \mathcal{F}_{k_{l}}\left(a_{1} \otimes \cdots \otimes a_{k}\right)\right. \\
& \left.+\sum_{j=1}^{j=k_{l}-1} \mathcal{F}_{k_{l}} \otimes \mathscr{F}_{k_{1}} \otimes \cdots \otimes \mathscr{F}_{k_{l-1}}\left(\tau^{j}\left(a_{1} \otimes \cdots \otimes a_{k}\right)\right)\right] .
\end{aligned}
$$

We leave the verification that $\tilde{F}_{\text {Hoch }}$ respects the Hochschild differential to the reader.

Let $\mathcal{A}$ be a dg algebra. Let $V^{\bullet}$ be a finite dimensional graded $\mathbb{C}$-vector space with 0 differential. Suppose that $\mathcal{F}$ is an $A_{\infty}$-morphism from $\mathcal{A}$ to $\operatorname{End}\left(V^{\bullet}\right)$ with Taylor components $\widetilde{F}_{k}$. Recall that for any $k>0$, we have a map $\tau: \mathcal{A}^{\otimes k} \rightarrow \mathcal{A}^{\otimes k}$ such that $\tau\left(a_{1} \otimes \cdots \otimes a_{k}\right)=(-1)^{\left(d_{k}+1\right)\left(d_{1}+\cdots+d_{k-1}+k-1\right)} a_{k} \otimes a_{1} \otimes \cdots \otimes a_{k-1}$ for homogenous elements $a_{1}, \ldots, a_{k}$ of $\mathcal{A}$ of degrees $d_{1}, \ldots, d_{k}$, respectively. By Proposition 1, str is a Hochschild 0-cocycle of $\operatorname{End}\left(V^{\bullet}\right)$. We now have the following corollary of Proposition 2.

Corollary 2. The supertrace on $\operatorname{End}\left(V^{\bullet}\right)$ pulls back to a Hochschild 0-cocycle tr of $\mathcal{A}$. On Hochschild 0 -cycles of $\mathcal{A}$ that arise from elements on degree $k-1$ in $\mathcal{A}^{\otimes k}$, the Hochschild cocycle tr is given by the map from $\mathcal{A}^{\otimes k}$ to $\mathbb{C}$ defined by

$$
a_{1} \otimes \cdots \otimes a_{k} \leadsto \sum_{j=0}^{j=k-1} \operatorname{str}\left(\mathcal{F}_{k}\left(\tau^{j}\left(a_{1} \otimes \cdots \otimes a_{k}\right)\right)\right) .
$$

Proof. The Hochschild cocycle tr is given by $\operatorname{tr}(x)=\operatorname{str}\left(\mathcal{F}_{\text {Hoch }}(x)\right)$ for any $x \in$ $C^{0}(\mathcal{A})$. Note that by Proposition $2, \mathscr{F}_{\text {Hoch }}(x) \in C^{0}\left(\operatorname{End}\left(V^{\bullet}\right)\right)$. The exact formula for $\operatorname{tr}$ given in this corollary is now immediate from the formula for $\widetilde{F}_{\text {Hoch }}$ given in the proof of Proposition 2. 


\section{The completed Hochschild homology of $\mathscr{D i f f}(\mathscr{E})$ and a linear functional on $\widehat{\mathbf{H H}}_{\mathbf{0}}(\mathscr{D i f f}(\mathcal{E}))$}

3.1. The basic construction in [FLS]. Let $K_{\dot{\mathcal{E}}}^{\bullet}$ denote the Dolbeaux complex of $\mathcal{E}$, as in the introduction to this article. Then $K_{\mathcal{E}}^{\bullet}$ decomposes as the direct sum of a complex with zero differential and an acyclic complex, i.e., $K_{\mathscr{E}}^{\bullet}=K_{0 \mathscr{E}}^{\bullet} \oplus K_{1 \mathcal{E}}^{\bullet}$ where $\mathrm{H}^{\bullet}\left(K_{0 \mathcal{E}}^{\bullet}\right)=\mathrm{H}^{\bullet}\left(K_{\mathcal{E}}^{\bullet}\right), K_{0 \mathcal{E}}^{\bullet}$ has 0 differential and $K_{1 \mathcal{E}}^{\bullet}$ is acyclic. This is a consequence of Hodge theory (see, for instance, Theorem 5.24 in [Vois]).

Recall that $\operatorname{Diff}^{\bullet}(\mathcal{E})=\operatorname{Dolb}^{\bullet}\left(X, \mathcal{O}_{X}\right) \otimes_{\mathcal{O}_{X}} \operatorname{Diff}(\mathcal{E})$ and that

$$
\operatorname{Diff}^{\bullet}(\mathscr{E}):=\Gamma\left(X, \operatorname{Diff}^{\bullet}(\mathcal{E})\right) .
$$

The main construction in $[\mathrm{FLS}]$ is that of an $A_{\infty}$-morphism $\mathcal{F}$ from $\operatorname{Diff}^{\bullet}(\mathcal{E})$ to $\operatorname{End}\left(K_{0 \mathcal{E}}^{\bullet}\right)$. Note that $K_{0 \mathcal{E}}^{\bullet}$ is a finite dimensional $\mathbb{C}$-vector space with 0 differential. As $\mathbb{C}$-vector spaces, $\mathrm{K}_{0 \mathscr{E}}^{i} \simeq \mathrm{H}^{i}(X, \mathcal{E})$. We may therefore apply Propositions 1,2 and Corollary 2, with $\mathcal{A}=\operatorname{Diff}^{\bullet}(\mathscr{E})$ and $\mathscr{B}=\operatorname{End}\left(K_{0 \mathscr{E}}^{\bullet}\right)$. We obtain the following facts immediately.

Fact 1 . By Proposition $1, \mathrm{HH}_{i}\left(\operatorname{End}\left(K_{0 \mathcal{E}}^{\bullet}\right)\right)=0$ for $i \neq 0$ and $\mathrm{HH}_{0}\left(\operatorname{End}\left(K_{0 \mathcal{E}}^{\bullet}\right)\right) \simeq \mathbb{C}$. This isomorphism is induced by the map taking a degree 0 element of $\operatorname{End}\left(K_{0 \mathcal{E}}^{\bullet}\right)$ to its supertrace.

Fact 2. By Proposition 2, the supertrace on $\operatorname{End}\left(K_{0 \varepsilon}^{\bullet}\right)$ pulls back to a Hochschild 0 -cocycle $\operatorname{tr}$ on $\operatorname{Diff}^{\bullet}(\mathcal{E})$. If $D$ is a Hochschild 0 -cycle of $\operatorname{Diff}^{\bullet}(\mathcal{E})$ that arises out of a degree $k-1$ element of $\operatorname{Diff}^{\bullet}(\mathcal{E})^{\otimes k}$, and if $[D]$ denotes the class of $D$ in $\mathrm{HH}_{0}\left(\right.$ Diff $\left.^{\bullet}(\mathcal{E})\right)$, then

$$
\operatorname{tr}([D])=\sum_{j=0}^{j=k-1} \operatorname{str}\left(\mathcal{F}_{k}\left(\tau^{j}(D)\right)\right) .
$$

Notation. We shall also denote the map

$$
D \mapsto \sum_{j=0}^{j=k-1} \operatorname{str}\left(\widetilde{F}_{k}\left(\tau^{j}(D)\right)\right)
$$

by $I_{\mathrm{FLS}}$.

3.1.1. Construction of $\mathfrak{F}$. We now recall the construction of $\mathscr{F}$ from [FLS]. Let $C_{k}$ denote the configuration space $\left\{t_{1}<\cdots<t_{k} \mid t_{i} \in \mathbb{R}\right\} / G^{(1)}$ where $G^{(1)}$ is the one-dimensional group of shifts $\left(t_{1}, \ldots, t_{k}\right) \rightarrow\left(t_{1}+c, \ldots, t_{k}+c\right)$. This is a smooth $(k-1)$-dimensional manifold though it is not compact if $k \geq 2$. Let $\tau_{i}=t_{i+1}-t_{i}$ for $1 \leq i \leq k-1$. The map $\left(t_{1}, \ldots, t_{k}\right) \sim\left(\tau_{1}, \ldots, \tau_{k-1}\right)$ is a diffeomorphism between $C_{k}$ and the product $\prod_{i=1}^{i=k-1}\left\{\tau_{i}>0\right\}$. Let $\left\{\overline{\tau_{i}>0}\right\}$ denote the compactification of 
$\left\{\tau_{i} \geq 0\right\}$ by a point at infinity. The cube $\left.\overline{C_{k}}:=\prod_{i=1}^{i=k-1} \overline{\left\{\tau_{i}>0\right.}\right\}$ is a compactification of $C_{k}$.

Let $D=D_{1} \otimes \cdots \otimes D_{k} \in \operatorname{Diff}^{\bullet}(\mathcal{E})^{\otimes k}$. Recall that the $D_{i}$ yield endomorphisms of $K_{\mathscr{E}}^{\bullet}$ as follows: Let $U \subset X$ be an open ball on which $\mathcal{E}$ is trivial. Let $z_{1}, \ldots, z_{n}$ be local holomorphic coordinates on $U$. A section of $\left.K_{\mathcal{E}}^{\bullet}\right|_{U}$ is a linear combination of sections of the form $s \otimes \overline{d z_{i_{1}}} \wedge \cdots \wedge \overline{d z_{i_{m}}} . D_{i}\left(s \otimes \overline{d z_{i_{1}}} \wedge \cdots \wedge \overline{d z_{i_{m}}}\right)=D_{i}(s) \otimes \overline{d z_{i_{1}}} \wedge \cdots \wedge \overline{d z_{i_{m}}}$.

Let $\overline{\partial \mathcal{E}}^{*}$ be the Hodge adjoint of $\overline{\partial_{\mathcal{E}}}$. Let $\Delta_{\mathcal{E}}$ denote the Laplacian of $\overline{\partial_{\mathcal{E}}}$. We also note that $\overline{\partial_{\mathcal{E}}}, \bar{\partial}_{\mathcal{E}}^{*}$ and $\Delta_{\mathcal{E}}$ yield endomorphisms of $K_{\mathcal{E}}^{\cdot}$.

Let $\Omega_{D}$ denote the differential form on $C_{k}$ with values in $\operatorname{End}\left(K_{0 \mathcal{E}}^{\cdot}\right)$ given by the formula

$$
\Omega_{D}=\Pi_{K_{0 \mathcal{E}}} \circ D_{k} \circ \exp \left[-d\left(t_{k}-t_{k-1}\right) \overline{\partial \mathcal{E}}^{*}-\left(t_{k}-t_{k-1}\right) \Delta_{\mathcal{E}}\right] \circ \cdots \circ D_{1} \circ \mathcal{I}_{K_{0 \mathcal{E}}}
$$

where $\Pi_{K_{0 \mathcal{E}}^{\bullet}}$ and $\mathcal{I}_{K_{0 \mathcal{E}}^{\bullet}}$ denote the projection from $K_{\mathcal{E}}^{\bullet}$ to $K_{0 \mathcal{E}}^{\bullet}$ and the inclusion from $K_{0 \mathcal{E}}^{\bullet}$ to $K_{\mathcal{E}}^{\bullet}$ respectively.

As noted in [FLS], to write $\Omega_{D}$ this way, we require that $\Delta_{\mathcal{E}}$ have discrete non negative spectrum (which is the case for a compact complex manifold). Further, as noted in [FLS], $\Omega_{D}$ extends to a $\operatorname{End}\left(K_{0 \mathcal{E}}^{\bullet}\right)$-valued (non-homogenous) differential form on $\overline{C_{k}}$.

We define

$$
\widetilde{F}_{k}(D)=\int_{C_{k}} \Omega_{D}=\int_{\overline{C_{k}}} \Omega_{D}
$$

By the integral over $\overline{C_{k}}$ of a non-homogenous differential form we mean the integral over $\overline{C_{k}}$ of its component of top de Rham degree. It helps to view the differential forms above as differential forms on $\overline{C_{k}}$ rather than on $C_{k}$ as that will ensure that the integrals defining the $\mathscr{F}_{k}$ 's converge.

That the $\widetilde{F}_{k}$ 's defined in this manner are the Taylor coefficients of an $A_{\infty}$-morphism is shown in [FLS].

Before we proceed further, we note that if $k=1$, then $C_{1}$ is a point. The formula for $\mathscr{F}_{k}$ given here yields that

$$
\widetilde{F}_{1}(D)=\Pi_{K_{0 \mathcal{E}}^{\bullet}} \circ D \circ \mathcal{I}_{K_{0 \mathcal{E}}}
$$

for $D \in \operatorname{Diff}^{\bullet}(\mathcal{E})$. In particular, if $\mathscr{D} \in \operatorname{Diff}(\mathcal{E})$ and if $\mathscr{D}_{*} \in \operatorname{End}\left(\mathrm{H}^{*}(X, \mathcal{E})\right)$ is the endomorphism of $\mathrm{H}^{*}(X, \mathcal{E})$ induced by $\mathscr{D}$, then $\mathcal{F}_{1}(\mathscr{D})=\mathscr{D}_{*}$. It follows from Fact 2 on p. 414 that $\operatorname{tr}([\mathscr{D}])=\operatorname{str}\left(\mathscr{D}_{*}\right)$. This proves that the following diagram commutes:

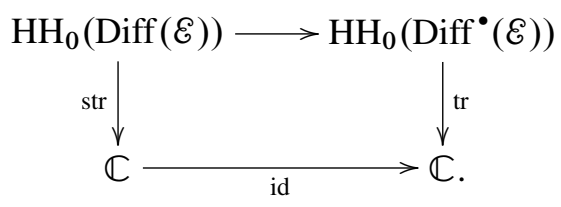


3.1.2. Rewriting the formula for $\widetilde{F}_{\boldsymbol{k}}$. Given an endomorphism $\varphi$ of $K_{\mathcal{E}}^{\bullet}$, let $[\varphi]_{i}$ denote the endomorphism id $\otimes \cdots \otimes \varphi \otimes \cdots \otimes$ id of $K_{\mathcal{E}}^{\bullet \otimes k}$ with $\varphi$ acting on the $i$-th factor from the right.

Let

$$
\begin{gathered}
\Phi=[\mathrm{id}]_{k} \circ\left[\exp \left[-d\left(t_{k}-t_{k-1}\right){\overline{\partial_{\mathcal{E}}}}^{*}-\left(t_{k}-t_{k-1}\right) \Delta_{\mathcal{E}}\right]\right]_{k-1} \circ \cdots \\
\cdots \circ\left[\exp \left[-d\left(t_{2}-t_{1}\right){\overline{\partial_{\mathcal{E}}}}^{*}-\left(t_{2}-t_{1}\right) \Delta_{\mathcal{E}}\right]\right]_{1} .
\end{gathered}
$$

This is a differential form on $C_{k}$ with values in $\operatorname{End}\left(K_{\mathcal{E}}^{\bullet \otimes k}\right)$, though it is not a differential operator. Similarly, if $D=D_{1} \otimes \cdots \otimes D_{k} \in \operatorname{Diff}^{\bullet}(\mathcal{E})^{\otimes k}, D$ yields an endomorphism $D:=\left[D_{1}\right]_{k} \circ \cdots \circ\left[D_{k}\right]_{1}$ of $\operatorname{End}\left(K_{\mathcal{E}}^{\bullet \otimes k}\right)$.

We have a composition map $m: \operatorname{End}\left(K_{\mathcal{E}}^{\bullet}\right)^{\otimes k} \rightarrow \operatorname{End}\left(K_{\mathcal{E}}^{\bullet}\right)$ Identifying $\operatorname{End}\left(K_{\mathcal{E}}^{\bullet \otimes k}\right)$ with $\operatorname{End}\left(K_{\mathcal{E}}^{\bullet}\right)^{\otimes k}$, we obtain a composition map $m: \operatorname{End}\left(K_{\mathcal{E}}^{\bullet \otimes k}\right) \rightarrow \operatorname{End}\left(K_{\mathcal{E}}^{\bullet}\right)$. We recall from [FLS] that the formula for $\Omega_{D}$ can be rewritten as follows:

$$
\Omega_{D}=\Pi_{K_{0 \varepsilon}} \circ m(\Phi \circ D) \circ I_{K_{0 \varepsilon}} \cdot
$$

Thus

$$
\mathcal{F}_{k}(D)=\int_{C_{k}} \Pi_{K_{0 \mathcal{E}}} \circ m(\Phi \circ D) \circ \mathcal{I}_{K_{0 \mathcal{E}}} \cdot
$$

3.2. Extending the supertrace $-I$. Recall that $\mathscr{D i f f}(\mathscr{E})$ denotes the sheaf of holomorphic differential operators on $\mathcal{E}$. Let $\operatorname{Diff}(\mathcal{E})(U)$ denote $\Gamma(U, \mathcal{D i f f}(\mathcal{E}))$ for any open $U \subset X$. Let $\mathcal{E}^{\bowtie k}$ denote the $k$-fold exterior tensor power of $\mathcal{E}$ on $X^{k}:=X \times$ $\cdots \times X$. We observe that the differential on the Hochschild complex $C^{\bullet}(\operatorname{Diff}(\mathscr{E})(U))$ extends to a differential on the graded vector space $\bigoplus_{k \geq 1} \operatorname{Diff}\left(\mathcal{E}^{\square k}\left(U^{k}\right)\right)[k-1]$. The resulting complex is called the completed Hochschild complex of $\mathscr{D i f f}(\mathscr{E})(U)$ and denoted by $\overline{C^{\bullet}(\operatorname{Diff}(\mathcal{E})(U))}$.

Definition 5. The completed Hochschild complex of $\mathscr{D i f f}(\mathscr{E})$ is the sheaf of complexes associated to the presheaf $\left.U \leadsto \overline{C^{\bullet}(\mathscr{D i f f}(\mathscr{E})(U)}\right)$ of complexes of $\mathbb{C}$-vector spaces.

The completed Hochschild complex of $\operatorname{Diff}(\mathscr{E})$ is a sheaf of complexes of $\mathbb{C}$ vector spaces on $X$. It is denoted in this article by $\widehat{\operatorname{Hoch}}(\mathscr{D i f f}(\mathcal{E}))$.

Definition 6. The $i$-th completed Hochschild homology $\widehat{\mathrm{HH}}_{i}(\mathscr{D i f f}(\mathcal{E}))$ of $\mathscr{D i f f}(\mathscr{E})$ is the hypercohomology $\mathbb{\boxplus}^{i}(X, \widehat{\operatorname{Hoch}}(\operatorname{Diff}(\mathcal{E})))$ of $\widehat{\operatorname{Hoch}}(\mathscr{D i f f}(\mathcal{E}))$.

Note that the $A_{\infty}$-map $\mathscr{F}$ whose construction we recalled in Section 3.1 enables us to pull back the supertrace on $\operatorname{End}\left(K_{\mathscr{E}}^{\bullet}\right)$ to a Hochschild 0-cocycle of $\operatorname{Diff}^{\bullet}(\mathcal{E})$. 
Unfortunately, the $A_{\infty}$-map $\mathscr{F}$ does not automatically enable us to directly pull back the supertrace to a $\mathbb{C}$-linear functional on $\widehat{\mathrm{HH}}_{0}(\mathscr{D i f f}(\mathscr{E}))$. This subsection is devoted to an important intermediate step that enables us to construct a $\mathbb{C}$-linear functional on $\widehat{\mathrm{HH}}_{0}(\mathscr{D i f f}(\mathcal{E}))$ which extends the supertrace on $\operatorname{Diff}(\mathscr{E})$.

3.2.1. "Estimating" $\boldsymbol{I}_{\text {FLS }}$. We now use a homotopy very similar to the homotopy in Proposition 3.1 [Tel] to show that the FLS functional of any 0-cycle in $C^{\bullet}\left(\operatorname{Diff}^{\bullet}(\mathcal{E})\right)$ depends only on its component in $\operatorname{Diff}^{0}(\mathscr{E})$.

Construction 1. Let $\phi: X \times X \rightarrow[0, \infty)$ be a Riemannian distance. Let $t$ be any positive real number. We can choose a finite cover of $X$ by open sets $U_{i}, 1 \leq i \leq m$, such that $\phi(x, y)<t$ for all $x, y \in U_{i}$ for any $i$. Choose a partition of unity $\left\{f_{i}\right\}$ by compactly supported (nonnegative valued) smooth functions subordinate to the cover $\left\{U_{i}\right\}$. Let $g_{i}$ be a compactly supported smooth function on $U_{i}$ with values in $[0,1]$ that is identically 1 on the support of $f_{i}$. Then $f:=\sum_{i=1}^{i=m} f_{i} \otimes g_{i}$ is a smooth function on $X \times X$ whose restriction to the diagonal is identically 1 . Also $f$ vanishes outside the subset $\{\phi(x, y) \leq t\}$ of $X \times X$, and the maximum value of $f$ on $X \times X$ is 1 .

Somewhat as in [Tel], let $E_{f}: \operatorname{Diff}^{\bullet}(\mathcal{E})^{\otimes k} \rightarrow \operatorname{Diff}^{\bullet}(\mathscr{E})^{\otimes k+1}$ be the map

$$
D_{1} \otimes \cdots \otimes D_{k} \mapsto-\sum_{i=1}^{i=m} f_{i} \otimes g_{i} D_{1} \otimes D_{2} \otimes \cdots \otimes D_{k} .
$$

Let $d$ be the differential of the complex $C^{\bullet}\left(\operatorname{Diff}^{\bullet}(\mathcal{E})\right)$. Then

$$
d E_{f}+E_{f} d=1-N_{f},
$$

where

$$
\begin{aligned}
& N_{f}\left(D_{1} \otimes \cdots \otimes D_{k}\right) \\
& = \pm \sum_{i=1}^{i=m} \bar{\partial} f_{i} \otimes g_{i} D_{1} \otimes \cdots \otimes D_{k} \pm \sum_{i=1}^{i=m} f_{i} \otimes\left(\bar{\partial} g_{i}\right) D_{1} \otimes \cdots \otimes D_{k} \\
& \quad \pm \sum_{i=1}^{i=m} f_{i} \otimes g_{i} D_{k} D_{1} \otimes \cdots \otimes D_{k-1} \pm \sum_{i=1}^{i=m} D_{k} f_{i} \otimes g_{i} D_{1} \otimes \cdots \otimes D_{k-1}
\end{aligned}
$$

for all $k \geq 2$.

Basic argument. Note that if $\alpha$ is a cycle in $C^{\bullet}\left(\operatorname{Diff}^{\bullet}(\mathcal{E})\right)$, then $\alpha$ is homotopic to $N_{f} \alpha$. This is true for all $t>0$. Also the FLS linear functional $I_{\mathrm{FLS}}$ is a Hochschild 0 -cocycle of $\operatorname{Diff}^{\bullet}(\mathcal{E})$. It follows that

$$
I_{\mathrm{FLS}}(\alpha)=I_{\mathrm{FLS}}\left(N_{f} \alpha\right) .
$$


Let $\alpha=\alpha_{1}+\cdots+\alpha_{p}$ with $\alpha_{i} \in \operatorname{Diff}^{\bullet}(\mathcal{E})^{\otimes i}$. We then show that

$$
\sum_{i \geq 2}\left|I_{\mathrm{FLS}}\left(N_{f} \alpha_{i}\right)\right| \leq C \epsilon(t),
$$

where $C$ is a constant that only depends on $\alpha$ and $\epsilon(t) \cdot \operatorname{vol}(X \times X)$ is the volume of the subset $\{\phi(x, y) \leq t\}$ of $X \times X$. Similarly, we show that $\left|I_{\mathrm{FLS}}\left(\alpha_{1}\right)-I_{\mathrm{FLS}}\left(N_{f}\left(\alpha_{1}\right)\right)\right| \leq$ $C^{\prime} \epsilon(t)$ where $C^{\prime}$ depends only on $\alpha$. Since the construction of $f$ as in Construction 1 is possible for all positive $t$, letting $t$ approach 0 we see that $I_{\mathrm{FLS}}(\alpha)=I_{\mathrm{FLS}}\left(\alpha_{1}\right)$.

Remark. Recall that $K_{0 \mathcal{E}}^{\bullet}$ can be identified with the kernel of the Laplacian $\Delta_{\mathcal{E}}$. Denote by $K_{L^{2} \mathcal{E}}^{\cdot}$ the Hilbert space of square integrable sections of $\operatorname{Dolb}^{\bullet}\left(X, \mathcal{O}_{X}\right) \otimes_{\mathcal{O}_{X}} \operatorname{Diff}(\mathcal{E})$. Then $\mathcal{I}_{K_{0 \mathcal{E}}^{\bullet}} \circ \Pi_{K_{0 \mathcal{E}}^{\bullet}}$ is an integral operator on $K_{L^{2} \mathcal{E}}^{\bullet}$ with smooth kernel that projects onto the image of $\mathcal{I}_{K_{0 \varepsilon}}$ (see [BGV], Chapter 2). We will denote this operator by $\Pi_{0, \mathcal{E}}$ or $\Pi_{0}$ when there is no confusion regarding the vector bundle being used. Also one can check that if $M \in \operatorname{End}\left(K_{\mathscr{E}}^{\bullet}\right)$, then $\Pi_{0} M \Pi_{0}$ makes sense as a trace class operator on $K_{L^{2} \varepsilon}^{\cdot}$ and

$$
\operatorname{str}\left(\Pi_{0} M \Pi_{0}\right)=\operatorname{str}\left(\Pi_{K_{0 \varepsilon}^{\bullet}} \circ M \circ \mathcal{I}_{K_{0 \varepsilon}^{\bullet}}\right) .
$$

Proposition 3. Let $D_{1}, \ldots, D_{k} \in \operatorname{Diff}^{\bullet}(\mathcal{E})$. Then

$$
\left|I_{\mathrm{FLS}}\left(N_{f}\left(D_{1} \otimes \cdots \otimes D_{k}\right)\right)\right| \leq C \epsilon(t) .
$$

The constant $C$ above depends only on $D_{1}, \ldots, D_{k}$.

Proof. Part 1: The setup.

Recall that

$$
\begin{aligned}
& N_{f}\left(D_{1} \otimes \cdots \otimes D_{k}\right) \\
&= \pm \sum_{i=1}^{i=m} \bar{\partial} f_{i} \otimes g_{i} D_{1} \otimes \cdots \otimes D_{k} \pm \sum_{i=1}^{i=m} f_{i} \otimes\left(\bar{\partial} g_{i}\right) D_{1} \otimes \cdots \otimes D_{k} \\
& \quad \pm \sum_{i=1}^{i=m} f_{i} \otimes g_{i} D_{k} D_{1} \otimes \cdots \otimes D_{k-1} \pm \sum_{i=1}^{i=m} D_{k} f_{i} \otimes g_{i} D_{1} \otimes \cdots \otimes D_{k-1}
\end{aligned}
$$

for all $k \geq 2$.

We estimate the FLS functional of each of the summands on the right-hand side separately. Let $\mathscr{F}_{k}$ denote the $k$-th Taylor component of the FLS $A_{\infty}$-map. Let $C_{k}$ denote the configuration space $\prod_{i=1}^{k-1}\left\{\tau_{i}>0\right\}$. Then if $\alpha_{i} \in \operatorname{Diff}^{\bullet}(\mathcal{E})$, it follows that

$$
\mathscr{F}_{k}\left(\alpha_{1} \otimes \cdots \otimes \alpha_{k}\right)=\int_{C_{k}} \Pi_{0} \alpha_{1} \bar{\partial}^{*} \mathrm{e}^{-\tau_{1} \Delta} \alpha_{2} \ldots \mathrm{e}^{-\tau_{k-1} \Delta} \alpha_{k} \Pi_{0} d \tau_{1} \ldots d \tau_{k-1} .
$$


Let $Q_{\tau}(x, y) \in \Gamma\left(X \times X, \mathcal{E} \otimes \Omega^{0, \bullet} \otimes\left(\Omega^{0, \bullet}\right)^{*} \otimes \mathcal{E}^{*}\right)$ denote the (smooth) kernel of the operator $\partial^{*} \mathrm{e}^{-\tau \Delta}$. Let $p_{\infty}(x, y)$ denote the (smooth) kernel of $\Pi_{0}$. Then the kernel of $\mathscr{F}_{k}\left(\alpha_{1} \otimes \cdots \otimes \alpha_{k}\right)$ is

$$
\begin{aligned}
\iint_{C_{k}} p^{k} & p_{\infty}\left(y_{0}, x_{1}\right) \alpha_{1, x_{1}} Q_{\tau_{1}}\left(x_{1}, x_{2}\right) \\
& \ldots Q_{\tau_{k-1}} \alpha_{k, x_{k}} p_{\infty}\left(x_{k}, y_{0}^{\prime}\right)\left|d x_{1}\right| \ldots\left|d x_{k}\right| d \tau_{1} \ldots d \tau_{k-1} .
\end{aligned}
$$

Recall that

$$
I_{\mathrm{FLS}}\left(\alpha_{1} \otimes \cdots \otimes \alpha_{k}\right)=\sum_{s=0}^{s=k-1} \operatorname{str}\left(\mathscr{F}_{k}\left(\sigma^{s}(\alpha)\right)\right)
$$

Here $\sigma$ is a (signed) cyclic permutation. Each of the $k$ cyclic permutations of $\alpha_{1} \otimes \cdots \otimes \alpha_{k}$ yields exactly one summand contributing towards $I_{\mathrm{FLS}}\left(\alpha_{1} \otimes \cdots \otimes \alpha_{k}\right)$.

Part 2: Estimating $I_{\mathrm{FLS}}\left( \pm \sum_{i=1}^{i=m} \bar{\partial} f_{i} \otimes g_{i} D_{1} \otimes \cdots \otimes D_{k}\right)$, I.

The cyclic permutations of $\sum_{i=1}^{i=m} \bar{\partial} f_{i} \otimes g_{i} D_{1} \otimes \cdots \otimes D_{k}$ are as follows:

$$
\begin{gathered}
\pm \sum_{i=1}^{i=m} \bar{\partial} f_{i} \otimes g_{i} D_{1} \otimes \cdots \otimes D_{k} \\
\pm \sum_{l=1}^{l=m} D_{k-i+1} \otimes \cdots \otimes D_{k} \otimes \bar{\partial} f_{l} \otimes g_{l} D_{1} \otimes \cdots \otimes D_{k-i}, \quad 1 \leq i \leq k-1 \\
\pm \sum_{l=1}^{l=m} g_{l} D_{1} \otimes \cdots \otimes D_{k} \otimes \bar{\partial} f_{l}
\end{gathered}
$$

We shall henceforth denote the kernel of an integral operator $T$ on $\Gamma\left(X, \mathcal{E} \otimes \Omega^{0, \bullet}(X)\right)$ by $\bar{T}$. Let $D^{a}$ denote the formal adjoint of a differential operator $D$ on $\Gamma\left(X, \mathcal{E} \otimes \Omega^{0, \bullet}(X)\right)$. This is a differential operator on $\Gamma\left(X, \Omega^{0,}(X)^{*} \otimes \mathcal{E}^{*}\right)$ since the bundle of densities on $X$ has a canonical trivialization (see [BGV], Chapter 2). With this in mind, we have

$$
\begin{gathered}
\overline{\mathcal{F}_{k+1}}\left(\sum_{i=1}^{i=m} \bar{\partial} f_{i} \otimes g_{i} D_{1} \otimes \cdots \otimes D_{k}\right) \\
=\int_{C_{k+1}} \int_{X^{k+1}} \sum_{i=1}^{i=m} p_{\infty}\left(y_{0}, x_{0}\right)\left(\bar{\partial} f_{i}\right)\left(x_{0}\right) Q_{\tau_{1}}\left(x_{0}, x_{1}\right) g_{i}\left(x_{1}\right) D_{1, x_{1}} Q_{\tau_{2}}\left(x_{1}, x_{2}\right) \ldots \\
\ldots p_{\infty}\left(x_{k}, y_{0}^{\prime}\right)\left|d x_{0}\right| \ldots\left|d x_{k}\right| d \tau_{1} \ldots d \tau_{k}
\end{gathered}
$$




$$
\begin{aligned}
&= \pm \int_{C_{k+1}} \int_{X^{k+1}} \sum_{i=1}^{i=m} p_{\infty}\left(y_{0}, x_{0}\right) \bar{\partial}_{x_{0}} f_{i}\left(x_{0}\right) Q_{\tau_{1}}\left(x_{0}, x_{1}\right) g_{i}\left(x_{1}\right) D_{1, x_{1}} Q_{\tau_{2}}\left(x_{1}, x_{2}\right) \ldots \\
& \pm \int_{C_{k+1}} \int_{X^{k+1}} \sum_{i=1}^{i=m} p_{\infty}\left(y_{0}, x_{0}\right) f_{i}\left(x_{0}\right) \bar{\partial}_{x_{0}} Q_{\tau_{1}}\left(x_{0}, x_{1}\right) g_{i}\left(x_{1}\right) D_{1, x_{1}} Q_{\tau_{2}}\left(x_{1}, x_{2}\right) \ldots \\
&= \pm \int_{C_{k+1}} \int_{X^{k+1}}\left[\bar{\partial}_{x_{0}}^{a} p_{\infty}\left(y_{0}, x_{0}\right)\right]\left[\sum_{i=1}^{i=m} f_{i}\left(x_{0}\right) g_{i}\left(x_{1}\right)\right]\left[Q_{\tau_{1}}\left(x_{0}, x_{1}\right)\right]\left[D_{1, x_{1}} Q_{\tau_{2}}\left(x_{1}, x_{2}\right)\right] \ldots \\
& \ldots\left[\ldots p_{\infty}\left(x_{k}, y_{0}^{\prime}\right)\right]\left|d x_{0}\right| \ldots\left|d x_{k}\right| d \tau_{1} \ldots d \tau_{k} \\
& \quad \int_{C_{k+1}} \int_{X^{k+1}}\left[p_{\infty}\left(y_{0}, x_{0}\right)\right]\left[\sum_{i=1}^{i=m} f_{i}\left(x_{0}\right) g_{i}\left(x_{1}\right)\right]\left[\bar{\partial}_{x_{0}} Q_{\tau_{1}}\left(x_{0}, x_{1}\right)\right]\left[D_{1, x_{1}} Q_{\tau_{2}}\left(x_{1}, x_{2}\right)\right] \ldots \\
& \ldots\left[\ldots p_{\infty}\left(x_{k}, y_{0}^{\prime}\right)\right]\left|d x_{0}\right| \ldots\left|d x_{k}\right| d \tau_{1} \ldots d \tau_{k} .
\end{aligned}
$$

The last equality in (2) is valid since $X$ is compact. Moreover, we also use the fact that if $h(x, y) \in \Gamma\left(X \times X, \mathcal{E} \otimes \Omega^{0, \bullet} \otimes \Omega^{0, \bullet *} \otimes \mathcal{E}^{*}\right)$, then it follows that $a(x) h(x, y) b(y)= \pm a(x) b(y) h(x, y)$ for smooth functions $a, b$ on $X$. We now note that if $p_{\tau}$ denotes the kernel of $e^{-\tau \Delta}$ then

$$
Q_{\tau}(x, y)=\bar{\partial}_{x}^{*} p_{\tau}(x, y)=\bar{\partial}^{*} p_{(0, \infty)}(x) p_{\tau}(x, y) p_{(0, \infty)}(y),
$$

where $p_{(0, \infty)}$ is the projection to the span of the eigenvectors of $\Delta$ corresponding to positive eigenvalues as in [BGV], Proposition 2.37. This is because $\bar{\partial}^{*}$ kills the kernel of $\Delta$. It follows from [BGV], Proposition 2.37, that

$$
\left\|Q_{\tau}(x, y)\right\|_{l} \leq C\left(\|\cdot\|_{l}\right) \mathrm{e}^{-\frac{1}{2} \lambda_{1} \tau}
$$

where $\|\cdot\|_{l}$ is any $C^{l}$ norm on $\Gamma\left(X \times X, \mathcal{E} \otimes \Omega^{0, \bullet} \otimes \Omega^{0,{ }^{*}} \otimes \mathcal{E}^{*}\right)$ and $\lambda_{1}$ is the first positive eigenvalue of $\Delta_{\mathcal{E}}$.

It follows from (3) that the sup-norm of each term within a square bracket in each integrand that involves a $Q_{\tau_{i}}$ is bounded above by $C_{i} \mathrm{e}^{-\tau_{i} \frac{\lambda_{1}}{2}}$ for some constant $C_{i}$ depending only on $D_{1} \otimes \cdots \otimes D_{k}$. The remaining terms have finite sup-norm. The sup-norm of $\sum_{i=1}^{i=m} f_{i}\left(x_{0}\right) g_{i}\left(x_{1}\right)$ is 1 . Hence there exists a positive constant $C$ depending only on $D_{1} \otimes \cdots \otimes D_{k}$ such that

$$
\begin{aligned}
& \left\|\overline{\mathcal{F}_{k+1}}\left(\sum_{i=1}^{i=m} \bar{\partial} f_{i} \otimes g_{i} D_{1} \otimes \cdots \otimes D_{k}\right)\right\|_{0} \\
& \quad \leq C \int_{C_{k+1}} \mathrm{e}^{-\tau_{1} \frac{\lambda_{1}}{2}} \ldots \mathrm{e}^{-\tau_{k} \frac{\lambda_{k}}{2}} \epsilon(t) \operatorname{vol}(X)^{k+1} d \tau_{1} \ldots d \tau_{k}=\frac{2^{k}}{\lambda_{1}^{k}} C \epsilon(t) \operatorname{vol}(X)^{k+1},
\end{aligned}
$$


where $\epsilon(t) \operatorname{vol}(X \times X)$ is the volume of the support of $\{\phi(x, y) \leq t\}$ in $X \times X$. Now

$$
\begin{aligned}
& \operatorname{str}\left(\widetilde{F}_{k+1}\left( \pm \sum_{i=1}^{i=m} \bar{\partial} f_{i} \otimes g_{i} D_{1} \otimes \cdots \otimes D_{k}\right)\right) \\
& \quad= \pm \int_{X} \operatorname{str}\left(\overline{\mathcal{F}}_{k+1}\left(\sum_{i=1}^{i=m} \bar{\partial} f_{i} \otimes g_{i} D_{1} \otimes \cdots \otimes D_{k}\right)\left(y_{0}, y_{0}\right)\left|d y_{0}\right|\right.
\end{aligned}
$$

It follows from (4) that

$$
\left|\operatorname{str}\left(\mathcal{F}_{k+1}\left( \pm \sum_{i=1}^{i=m} \bar{\partial} f_{i} \otimes g_{i} D_{1} \otimes \cdots \otimes D_{k}\right)\right)\right| \leq C_{1} \epsilon(t)
$$

for some constant $C_{1}$ depending on $D_{1} \otimes \cdots \otimes D_{k}$ only.

The same method is used to estimate the other summands that contribute to $\left.I_{\mathrm{FLS}}\left( \pm \sum_{i=1}^{i=m} \bar{\partial} f_{i} \otimes g_{i} D_{1} \otimes \cdots \otimes D_{k}\right)\right)$ and show that those contributions are at most $C^{\prime} \epsilon(t)$ as well - in fact the FLS functionals of the other summands of $N_{f}\left(D_{1} \otimes \cdots \otimes D_{k}\right)$ are estimated in the same way. We however, present the detailed calculations in the next two parts to be extra careful.

Part 3: Estimating $\left.I_{\mathrm{FLS}}\left( \pm \sum_{i=1}^{i=m} \bar{\partial} f_{i} \otimes g_{i} D_{1} \otimes \cdots \otimes D_{k}\right)\right)$, II.

We show the equivalent of the calculation (2) for the other summands contributing to $I_{\mathrm{FLS}}\left( \pm \sum_{i=1}^{i=m} \frac{\partial}{\partial} f_{i} \otimes g_{i} D_{1} \otimes \cdots \otimes D_{k}\right)$. At the end of each calculation, the integrand in the integral computing the kernel of the operator whose supertrace we need is written as a product of terms marked by square brackets. The argument in part 2 then works almost word for word to show that the corresponding contribution to $I_{\mathrm{FLS}}\left(N_{f}\left(D_{1} \otimes \cdots \otimes D_{k}\right)\right)$ is bounded by a constant depending on the $D_{i}$ 's times $\epsilon(t)$. We have

$$
\begin{aligned}
& \overline{\mathcal{F}_{k+1}}\left(\sum_{l=1}^{l=m} D_{k-i+1} \otimes \cdots \otimes D_{k} \otimes \bar{\partial} f_{l} \otimes g_{l} D_{1} \otimes \cdots \otimes D_{k-i}\right) \\
& =\int_{C_{k+1}} \int_{X^{k+1}} \sum_{l=1}^{l=m} p_{\infty}\left(y_{0}, x_{0}\right) D_{k-i+1, x_{0}} Q_{\tau_{1}}\left(x_{0}, x_{1}\right) \ldots \\
& \ldots D_{k, x_{i-1}} Q_{\tau_{i}}\left(x_{i-1}, x_{i}\right)\left(\bar{\partial} f_{l}\right)\left(x_{i}\right) Q_{\tau_{i+1}}\left(x_{i}, x_{i+1}\right) g_{l}\left(x_{i+1}\right) D_{1, x_{i+1}} \ldots \\
& \ldots p_{\infty}\left(x_{k}, y_{0}^{\prime}\right)\left|d x_{0}\right| \ldots\left|d x_{k}\right| d \tau_{1} \ldots d \tau_{k} \\
& = \pm \int_{C_{k+1}} \int_{X^{k+1}} \sum_{l=1}^{l=m} p_{\infty}\left(y_{0}, x_{0}\right) D_{k-i+1, x_{0}} Q_{\tau_{1}}\left(x_{0}, x_{1}\right) \ldots \\
& \left.\ldots D_{k, x_{i-1}} Q_{\tau_{i}}\left(x_{i-1}, x_{i}\right) \bar{\partial}_{x_{i}} f_{l}\left(x_{i}\right) Q_{\tau_{i+1}}\left(x_{i}, x_{i+1}\right) g_{l} x_{i+1}\right) D_{1, x_{i+1}} \ldots \\
& \ldots p_{\infty}\left(x_{k}, y_{0}^{\prime}\right)\left|d x_{0}\right| \ldots\left|d x_{k}\right| d \tau_{1} \ldots d \tau_{k} \\
& \pm \int_{C_{k+1}} \int_{X^{k+1}} \sum_{l=1}^{l=m} p_{\infty}\left(y_{0}, x_{0}\right) D_{k-i+1, x_{0}} Q_{\tau_{1}}\left(x_{0}, x_{1}\right) \ldots \\
& \ldots D_{k, x_{i-1}} Q_{\tau_{i}}\left(x_{i-1}, x_{i}\right) f_{l}\left(x_{i}\right) \bar{\partial}_{x_{i}} Q_{\tau_{i+1}}\left(x_{i}, x_{i+1}\right) g_{l}\left(x_{i+1}\right) D_{1, x_{i+1}} \ldots \\
& \ldots p_{\infty}\left(x_{k}, y_{0}^{\prime}\right)\left|d x_{0}\right| \ldots\left|d x_{k}\right| d \tau_{1} \ldots d \tau_{k}
\end{aligned}
$$




$$
\begin{aligned}
&= \pm \int_{C_{k+1}} \int_{X^{k+1}}\left[p_{\infty}\left(y_{0}, x_{0}\right)\right]\left[D_{k-i+1, x_{0}} Q_{\tau_{1}}\left(x_{0}, x_{1}\right)\right] \ldots \\
& \ldots\left[D_{k, x_{i-1}} \bar{\partial}_{x_{i}}^{a} Q_{\tau_{i}}\left(x_{i-1}, x_{i}\right)\right]\left[\sum_{l=1}^{l=m} f_{l}\left(x_{i}\right) g_{l}\left(x_{i+1}\right)\right]\left[Q_{\tau_{i+1}}\left(x_{i}, x_{i+1}\right)\right] \\
& \quad \cdot\left[D_{1, x_{i+1}} \ldots\right]\left[\ldots p_{\infty}\left(x_{k}, y_{0}^{\prime}\right)\right]\left|d x_{0}\right| \ldots\left|d x_{k}\right| d \tau_{1} \ldots d \tau_{k} \\
& \pm \quad \int_{C_{k+1}} \int_{X^{k+1}}\left[p_{\infty}\left(y_{0}, x_{0}\right)\right]\left[D_{k-i+1, x_{0}} Q_{\tau_{1}}\left(x_{0}, x_{1}\right)\right] \ldots \\
& \quad \ldots\left[D_{k, x_{i-1}} Q_{\tau_{i}}\left(x_{i-1}, x_{i}\right)\right]\left[\sum_{l=1}^{l=m} f_{l}\left(x_{i}\right) g_{l}\left(x_{i+1}\right)\right]\left[\bar{\partial}_{x_{i}} Q_{\tau_{i+1}}\left(x_{i}, x_{i+1}\right)\right] \\
& \\
& \quad \cdot\left[D_{1, x_{i+1}} \ldots\right]\left[\ldots p_{\infty}\left(x_{k}, y_{0}^{\prime}\right)\right]\left|d x_{0}\right| \ldots\left|d x_{k}\right| d \tau_{1} \ldots d \tau_{k}
\end{aligned}
$$

and

$$
\begin{aligned}
& \mathscr{F}_{k+1}\left(\sum_{l=1}^{l=m} g_{l} D_{1} \otimes \cdots \otimes D_{k} \otimes \bar{\partial} f_{l}\right) \\
& =\int_{C_{k+1}} \int_{X^{k+1}} \sum_{l=1}^{l=m} p_{\infty}\left(y_{0}, x_{0}\right) g_{l}\left(x_{0}\right) D_{1, x_{0}} Q_{\tau_{1}}\left(x_{0}, x_{1}\right) \ldots \\
& \ldots D_{k} Q_{\tau_{k}}\left(x_{k-1}, x_{k}\right)\left(\bar{\partial} f_{l}\right)\left(x_{k}\right) p_{\infty}\left(x_{k}, y_{0}^{\prime}\right)\left|d x_{0}\right| \ldots\left|d x_{k}\right| d \tau_{1} \ldots d \tau_{k} \\
& = \pm \int_{C_{k+1}} \int_{X^{k+1}} \sum_{l=1}^{l=m} p_{\infty}\left(y_{0}, x_{0}\right) g_{l}\left(x_{0}\right) D_{1, x_{0}} Q_{\tau_{1}}\left(x_{0}, x_{1}\right) \ldots \\
& \ldots D_{k} Q_{\tau_{k}}\left(x_{k-1}, x_{k}\right) \bar{\partial}_{x_{k}} f_{l}\left(x_{k}\right) p_{\infty}\left(x_{k}, y_{0}^{\prime}\right)\left|d x_{0}\right| \ldots\left|d x_{k}\right| d \tau_{1} \ldots d \tau_{k} \\
& \pm \int_{C_{k+1}} \int_{X^{k+1}} \sum_{l=1}^{l=m} p_{\infty}\left(y_{0}, x_{0}\right) g_{l}\left(x_{0}\right) D_{1, x_{0}} Q_{\tau_{1}}\left(x_{0}, x_{1}\right) \ldots \\
& \ldots D_{k} Q_{\tau_{k}}\left(x_{k-1}, x_{k}\right) f_{l}\left(x_{k}\right) \bar{\partial}_{x_{k}} p_{\infty}\left(x_{k}, y_{0}^{\prime}\right)\left|d x_{0}\right| \ldots\left|d x_{k}\right| d \tau_{1} \ldots d \tau_{k} \\
& = \pm \int_{C_{k+1}} \int_{X^{k+1}}\left[p_{\infty}\left(y_{0}, x_{0}\right)\right]\left[\sum_{l=1}^{l=m} f_{l}\left(x_{k}\right) g_{l}\left(x_{0}\right)\right]\left[D_{1, x_{0}} Q_{\tau_{1}}\left(x_{0}, x_{1}\right)\right] \ldots \\
& \ldots\left[D_{k} \bar{\partial}_{x_{k}}^{a} Q_{\tau_{k}}\left(x_{k-1}, x_{k}\right)\right]\left[p_{\infty}\left(x_{k}, y_{0}^{\prime}\right)\right]\left|d x_{0}\right| \ldots\left|d x_{k}\right| d \tau_{1} \ldots d \tau_{k} \\
& \pm \int_{C_{k+1}} \int_{X^{k+1}}\left[p_{\infty}\left(y_{0}, x_{0}\right)\right]\left[\sum_{l=1}^{l=m} f_{l}\left(x_{k}\right) g_{l}\left(x_{0}\right)\right]\left[D_{1, x_{0}} Q_{\tau_{1}}\left(x_{0}, x_{1}\right)\right] \ldots \\
& \ldots\left[D_{k} Q_{\tau_{k}}\left(x_{k-1}, x_{k}\right)\right]\left[\bar{\partial}_{x_{k}} p_{\infty}\left(x_{k}, y_{0}^{\prime}\right)\right]\left|d x_{0}\right| \ldots\left|d x_{k}\right| d \tau_{1} \ldots d \tau_{k} .
\end{aligned}
$$

Part 4: Estimating other summands contributing to $I_{\mathrm{FLS}}\left(N_{f}\left(D_{1} \otimes \cdots \otimes D_{k}\right)\right)$. 
Recall that

$$
\begin{aligned}
& N_{f}\left(D_{1} \otimes\right.\left.\cdots \otimes D_{k}\right) \\
&= \pm \sum_{i=1}^{i=m} \bar{\partial} f_{i} \otimes g_{i} D_{1} \otimes \cdots \otimes D_{k} \pm \sum_{i=1}^{i=m} f_{i} \otimes\left(\bar{\partial} g_{i}\right) D_{1} \otimes \cdots \otimes D_{k} \\
& \quad \pm \sum_{i=1}^{i=m} f_{i} \otimes g_{i} D_{k} D_{1} \otimes \cdots \otimes D_{k-1} \pm \sum_{i=1}^{i=m} D_{k} f_{i} \otimes g_{i} D_{1} \otimes \cdots \otimes D_{k-1}
\end{aligned}
$$

for all $k \geq 2$.

The cyclic permutations of $\sum_{l=1}^{l=m} f_{l} \otimes g_{l} D_{k} D_{1} \otimes \cdots \otimes D_{k-1}$ that contribute to its FLS functional are as follows:

$$
\begin{gathered}
\sum_{l=1}^{l=m} f_{l} \otimes g_{l} D_{k} D_{1} \otimes \cdots \otimes D_{k-1}, \\
D_{k-i} \otimes \cdots \otimes D_{k-1} \otimes \sum_{l=1}^{l=m} f_{l} \otimes g_{l} D_{k} D_{1} \otimes \cdots \otimes D_{k-i-1}, \quad 1 \leq i \leq k-2, \\
\sum_{l=1}^{l=m} g_{l} D_{k} D_{1} \otimes \cdots \otimes D_{k-1} \otimes f_{l} .
\end{gathered}
$$

As before we have

$$
\begin{gathered}
\overline{\mathcal{F}_{k}}\left(\sum_{l=1}^{l=m} f_{l} \otimes g_{l} D_{k} D_{1} \otimes \cdots \otimes D_{k-1}\right) \\
=\int_{C_{k}} \int_{X^{k}}\left[p_{\infty}\left(y_{0}, x_{1}\right)\right]\left[\sum_{l=1}^{l=m} f_{l}\left(x_{1}\right) g_{l}\left(x_{2}\right)\right]\left[Q_{\tau_{1}}\left(x_{1}, x_{2}\right)\right]\left[D_{k, x_{2}} D_{1, x_{2}} \ldots\right] \ldots \\
\ldots\left[D_{k-1} p_{\infty}\left(x_{k}, y_{0}^{\prime}\right)\right]\left|d x_{1}\right| \ldots\left|d x_{k}\right| d \tau_{1} \ldots d \tau_{k-1}, \\
\overline{\mathcal{F}_{k}}\left(D_{k-i} \otimes \cdots \otimes D_{k-1} \otimes \sum_{l=1}^{l=m} f_{l} \otimes g_{l} D_{k} D_{1} \otimes \cdots \otimes D_{k-i-1}\right) \\
=\int_{C_{k}} \int_{X^{k}}\left[p_{\infty}\left(y_{0}, x_{1}\right)\right]\left[D_{k-i, x_{1}} Q_{\tau_{1}}\left(x_{1}, x_{2}\right)\right] \ldots \\
\quad \ldots\left[\sum_{l=1}^{l=m} f_{l}\left(x_{i+1}\right) g_{l}\left(x_{i+2}\right)\right]\left[Q_{\tau_{i+1}}\left(x_{i+1}, x_{i+2}\right)\right]\left[D_{k, x_{i+2}} D_{1, x_{i+2}} \ldots\right] \ldots \\
\quad\left[\ldots p_{\infty}\left(x_{k}, y_{0}^{\prime}\right)\right]\left|d x_{1}\right| \ldots\left|d x_{k}\right| d \tau_{1} \ldots d \tau_{k-1}
\end{gathered}
$$


and

$$
\begin{gathered}
{\overline{\mathcal{F}_{k}}}\left(\sum_{l=1}^{l=m} g_{l} D_{k} D_{1} \otimes \cdots \otimes D_{k-1} \otimes f_{l}\right) \\
=\int_{C_{k}} \int_{X^{k}}\left[p_{\infty}\left(y_{0}, x_{1}\right)\right]\left[\sum_{l=1}^{l=m} f_{l}\left(x_{k}\right) g_{l}\left(x_{1}\right)\right]\left[D_{k, x_{1}} D_{1, x_{1}} Q_{\tau_{1}}\left(x_{1}, x_{2}\right)\right] \ldots \\
\ldots\left[p_{\infty}\left(x_{k}, y_{0}^{\prime}\right)\right]\left|d x_{1}\right| \ldots\left|d x_{k}\right| d \tau_{1} \ldots d \tau_{k-1}
\end{gathered}
$$

For each of the summands dealt with by equations (7), (8) and (9) respectively, one can argue exactly as we did after (2).

The cyclic permutations of $\sum_{l=1}^{l=m} D_{k} f_{l} \otimes g_{l} D_{1} \otimes \cdots \otimes D_{k-1}$ that contribute to its FLS functional are

$$
\begin{gathered}
\sum_{l=1}^{l=m} D_{k} f_{l} \otimes g_{l} D_{1} \otimes \cdots \otimes D_{k-1}, \\
\sum_{l=1}^{l=m} D_{k-i} \otimes \cdots \otimes D_{k-1} \otimes D_{k} f_{l} \otimes g_{l} D_{1} \otimes \cdots \otimes D_{k-i-1}, \quad 1 \leq i \leq k-2, \\
\sum_{l=1}^{l=m} g_{l} D_{1} \otimes \cdots \otimes D_{k} f_{l} .
\end{gathered}
$$

We have

$$
\begin{aligned}
& \overline{\mathcal{F}_{k}}\left(D_{k} f_{l} \otimes g_{l} D_{1} \otimes \cdots \otimes D_{k-1}\right) \\
& =\iint_{C_{k} X^{k}} \sum_{l=1}^{l=m} p_{\infty}\left(y_{0}, x_{1}\right) D_{k, x_{1}} f_{l}\left(x_{1}\right) Q_{\tau_{1}}\left(x_{1}, x_{2}\right) g_{l}\left(x_{2}\right) D_{1, x_{2}} \ldots \\
& \ldots D_{k-1, x_{k}} p_{\infty}\left(x_{k}, y_{0}^{\prime}\right)\left|d x_{1}\right| \ldots\left|d x_{k}\right| d \tau_{1} \ldots d \tau_{k-1} \\
& =\int_{C_{k}} \int_{X^{k}}\left[D_{k, x_{1}}^{a} p_{\infty}\left(y_{0}, x_{1}\right)\right]\left[\sum_{l=1}^{l=m} f_{l}\left(x_{1}\right) g_{l}\left(x_{2}\right)\right]\left[Q_{\tau_{1}}\left(x_{1}, x_{2}\right)\right]\left[D_{1, x_{2}} \ldots\right] \ldots \\
& \ldots\left[D_{k-1, x_{k}} p_{\infty}\left(x_{k}, y_{0}^{\prime}\right)\right]\left|d x_{1}\right| \ldots\left|d x_{k}\right| d \tau_{1} \ldots d \tau_{k-1}, \\
& \overline{\mathcal{F}}_{k}\left(\sum_{l=1}^{l=m} D_{k-i} \otimes \cdots \otimes D_{k-1} \otimes D_{k} f_{l} \otimes g_{l} D_{1} \otimes \cdots \otimes D_{k-i-1}\right) \\
& =\iint_{C_{k}} \sum_{l=1}^{l=m} p_{\infty}\left(y_{0}, x_{1}\right) D_{k-i, x_{1}} Q_{\tau_{1}}\left(x_{1}, x_{2}\right) \ldots \\
& \ldots D_{k-1, x_{i}} Q_{\tau_{i}}\left(x_{i}, x_{i+1}\right) D_{k, x_{i+1}} f_{l}\left(x_{i+1}\right) Q_{\tau_{i+1}}\left(x_{i+1}, x_{i+2}\right) g_{l}\left(x_{i+2}\right) \\
& \text {. } D_{1, x_{i+2}} \ldots p_{\infty}\left(x_{k}, y_{0}^{\prime}\right)\left|d x_{1}\right| \ldots\left|d x_{k}\right| d \tau_{1} \ldots d \tau_{k-1}
\end{aligned}
$$




$$
\begin{aligned}
=\iint_{C_{k}} \int^{k} & {\left[p_{\infty}\left(y_{0}, x_{1}\right)\right]\left[D_{k-i, x_{1}} Q_{\tau_{1}}\left(x_{1}, x_{2}\right)\right] \ldots } \\
\ldots & {\left[D_{k-1, x_{i}} D_{k, x_{i+1}}^{a} Q_{\tau_{i}}\left(x_{i}, x_{i+1}\right)\right]\left[\sum_{l=1}^{l=m} f_{l}\left(x_{i+1}\right) g_{l}\left(x_{i+2}\right)\right] } \\
\cdot & {\left[Q_{\tau_{i+1}}\left(x_{i+1}, x_{i+2}\right)\right]\left[D_{1, x_{i+2}} \ldots\right] \ldots\left[\ldots p_{\infty}\left(x_{k}, y_{0}^{\prime}\right)\right] } \\
\cdot & \left|d x_{1}\right| \ldots\left|d x_{k}\right| d \tau_{1} \ldots d \tau_{k-1}
\end{aligned}
$$

and

$$
\begin{gathered}
\overline{\mathcal{F}_{k}}\left(\sum_{l=1}^{l=m} g_{l} D_{1} \otimes \cdots \otimes D_{k} f_{l}\right) \\
=\int_{C_{k}} \int_{X^{k}} \sum_{l=1}^{l=m} p_{\infty}\left(y_{0}, x_{1}\right) g_{l}\left(x_{1}\right) D_{1, x_{1}} Q_{\tau_{1}}\left(x_{1}, x_{2}\right) \ldots \\
\quad \ldots D_{k-1, x_{k-1}} Q_{\tau_{k-1}}\left(x_{k-1}, x_{k}\right) D_{k, x_{k}} f_{l}\left(x_{k}\right) \\
\quad \cdot p_{\infty}\left(x_{k}, y_{0}^{\prime}\right)\left|d x_{1}\right| \ldots\left|d x_{k}\right| d \tau_{1} \ldots d \tau_{k-1} \\
=\int_{C_{k}} \int_{X^{k}}\left[p_{\infty}\left(y_{0}, x_{1}\right)\right]\left[\sum_{l=1}^{l=m} f_{l}\left(x_{k}\right) g_{l}\left(x_{1}\right)\right]\left[D_{1, x_{1}} Q_{\tau_{1}}\left(x_{1}, x_{2}\right)\right] \ldots \\
\quad \ldots\left[D_{k-1, x_{k-1}} D_{k, x_{k}}^{a} Q_{\tau_{k-1}}\left(x_{k-1}, x_{k}\right)\right] \\
\quad \cdot\left[p_{\infty}\left(x_{k}, y_{0}^{\prime}\right)\right]\left|d x_{1}\right| \ldots\left|d x_{k}\right| d \tau_{1} \ldots d \tau_{k-1} .
\end{gathered}
$$

For each of the summands dealt with by equations (10), (11) and (12) respectively, one can argue exactly as we did after (2).

The cyclic permutations of $\sum_{l=1}^{l=m} f_{l} \otimes\left(\bar{\partial} g_{l}\right) D_{1} \otimes \cdots \otimes D_{k}$ that contribute to its FLS functional are as follows:

$$
\sum_{l=1}^{l=m} f_{l} \otimes\left(\bar{\partial} g_{l}\right) D_{1} \otimes \cdots \otimes D_{k}
$$

$$
\sum_{l=1}^{l=m} D_{k-i} \otimes \cdots \otimes D_{k} \otimes f_{l} \otimes\left(\bar{\partial} g_{l}\right) D_{1} \otimes \cdots \otimes D_{k-i-1}, \quad 0 \leq i \leq k-2,
$$

$$
\sum_{l=1}^{l=m}\left(\bar{\partial} g_{l}\right) D_{1} \otimes \cdots \otimes D_{k} \otimes f_{l}
$$

We have

$$
\begin{gathered}
\overline{\mathcal{F}_{k+1}}\left(\sum_{l=1}^{l=m} f_{l} \otimes\left(\bar{\partial} g_{l}\right) D_{1} \otimes \cdots \otimes D_{k}\right) \\
=\int_{C_{k+1}} \int_{X^{k+1}} \sum_{l=1}^{l=m} p_{\infty}\left(y_{0}, x_{0}\right) f_{l}\left(x_{0}\right) Q_{\tau_{1}}\left(x_{0}, x_{1}\right)\left(\bar{\partial} g_{l}\right)\left(x_{1}\right) D_{1, x_{1}} \ldots \\
\ldots p_{\infty}\left(x_{k}, y_{0}^{\prime}\right)\left|d x_{0}\right| \ldots\left|d x_{k}\right| d \tau_{1} \ldots d \tau_{k}
\end{gathered}
$$




$$
\begin{aligned}
& = \pm \int_{C_{k+1}} \int_{X^{k+1}} \sum_{l=1}^{l=m} p_{\infty}\left(y_{0}, x_{0}\right) f_{l}\left(x_{0}\right) Q_{\tau_{1}}\left(x_{0}, x_{1}\right) \bar{\partial}_{x_{1}} g_{l}\left(x_{1}\right) D_{1, x_{1}} \ldots \\
& \ldots p_{\infty}\left(x_{k}, y_{0}^{\prime}\right)\left|d x_{0}\right| \ldots\left|d x_{k}\right| d \tau_{1} \ldots d \tau_{k} \\
& \pm \int_{C_{k+1}} \int_{X^{k+1}} \sum_{l=1}^{l=m} p_{\infty}\left(y_{0}, x_{0}\right) f_{l}\left(x_{0}\right) Q_{\tau_{1}}\left(x_{0}, x_{1}\right) g_{l}\left(x_{1}\right) \bar{\partial}_{x_{1}} D_{1, x_{1}} \ldots \\
& \ldots p_{\infty}\left(x_{k}, y_{0}^{\prime}\right)\left|d x_{0}\right| \ldots\left|d x_{k}\right| d \tau_{1} \ldots d \tau_{k} \\
& = \pm \int_{C_{k+1}} \int_{X^{k+1}}\left[p_{\infty}\left(y_{0}, x_{0}\right)\right]\left[\sum_{l=1}^{l=m} f_{l}\left(x_{0}\right) g_{l}\left(x_{1}\right)\right]\left[\bar{\partial}_{x_{1}}^{a} Q_{\tau_{1}}\left(x_{0}, x_{1}\right)\right] \\
& \cdot\left[D_{1, x_{1}} \ldots\right] \ldots\left[\ldots p_{\infty}\left(x_{k}, y_{0}^{\prime}\right)\right]\left|d x_{0}\right| \ldots\left|d x_{k}\right| d \tau_{1} \ldots d \tau_{k} \\
& \pm \int_{C_{k+1}} \int_{X^{k+1}}\left[p_{\infty}\left(y_{0}, x_{0}\right)\right]\left[\sum_{l=1}^{l=m} f_{l}\left(x_{0}\right) g_{l}\left(x_{1}\right)\right]\left[Q_{\tau_{1}}\left(x_{0}, x_{1}\right)\right]\left[\bar{\partial}_{x_{1}}\right. \\
& \left.\cdot D_{1, x_{1}} \ldots\right] \ldots\left[\ldots p_{\infty}\left(x_{k}, y_{0}^{\prime}\right)\right]\left|d x_{0}\right| \ldots\left|d x_{k}\right| d \tau_{1} \ldots d \tau_{k},
\end{aligned}
$$

$\overline{\mathcal{F}_{k+1}}\left(\sum_{l=1}^{l=m} D_{k-i} \otimes \cdots \otimes D_{k} \otimes f_{l} \otimes\left(\bar{\partial} g_{l}\right) D_{1} \otimes \cdots \otimes D_{k-i-1}\right)$

$$
=\int_{C_{k+1}} \int_{X^{k+1}} \sum_{l=1}^{l=m} p_{\infty}\left(y_{0}, x_{0}\right) \ldots Q_{\tau_{i+1}}\left(x_{i}, x_{i+1}\right) f_{l}\left(x_{i+1}\right)
$$

$$
\begin{gathered}
\cdot Q_{\tau_{i+2}}\left(x_{i+1}, x_{i+2}\right)\left(\bar{\partial} g_{l}\right)\left(x_{i+2}\right) D_{1, x_{i+2}} \ldots \\
\ldots p_{\infty}\left(x_{k}, y_{0}^{\prime}\right)\left|d x_{0}\right| \ldots\left|d x_{k}\right| d \tau_{1} \ldots d \tau_{k} \\
= \pm \int_{C_{k+1}} \int_{X^{k+1}}^{l=m} p_{\infty}\left(y_{0}, x_{0}\right) \ldots Q_{\tau_{i+1}}\left(x_{i}, x_{i+1}\right) f_{l}\left(x_{i+1}\right) \\
\cdot Q_{\tau_{i+2}}\left(x_{i+1}, x_{i+2}\right) \bar{\partial}_{x_{i+2}} g_{l}\left(x_{i+2}\right) D_{1, x_{i+2}} \ldots \\
\ldots p_{\infty}\left(x_{k}, y_{0}^{\prime}\right)\left|d x_{0}\right| \ldots\left|d x_{k}\right| d \tau_{1} \ldots d \tau_{k} \\
\int_{C_{k+1} X^{k+1}} \sum_{l=1}^{l=m} p_{\infty}\left(y_{0}, x_{0}\right) \ldots Q_{\tau_{i+1}}\left(x_{i}, x_{i+1}\right) f_{l}\left(x_{i+1}\right) \\
\cdot Q_{\tau_{i+2}}\left(x_{i+1}, x_{i+2}\right) g_{l}\left(x_{i+2}\right) \bar{\partial}_{x_{i+2}} D_{1, x_{i+2}} \ldots \\
\ldots p_{\infty}\left(x_{k}, y_{0}^{\prime}\right)\left|d x_{0}\right| \ldots\left|d x_{k}\right| d \tau_{1} \ldots d \tau_{k} \\
=\int_{C_{k+1} X^{k+1}}\left[p_{\infty}\left(y_{0}, x_{0}\right)\right] \ldots\left[\ldots Q_{\tau_{i+1}}\left(x_{i}, x_{i+1}\right)\right]\left[\sum_{l=1}^{l=m} f_{l}\left(x_{i+1}\right) g_{l}\left(x_{i+2}\right)\right]
\end{gathered}
$$

$$
\cdot\left[\bar{\partial}_{x_{i+2}}^{a} Q_{\tau_{i+2}}\left(x_{i+1}, x_{i+2}\right)\right]\left[D_{1, x_{i+2}} \ldots\right] \ldots\left[\ldots p_{\infty}\left(x_{k}, y_{0}^{\prime}\right)\right]
$$




$$
\begin{aligned}
\pm \int_{C_{k+1}} \int_{X^{k+1}}\left[p_{\infty}\left(y_{0}, x_{0}\right)\right] \ldots\left[\ldots x_{\tau_{i+1}}\left(x_{i}, x_{i+1}\right)\right] \\
\cdot\left[\sum_{l=1}^{l=m} f_{l}\left(x_{i+1}\right) g_{l}\left(x_{i+2}\right)\right] \\
\cdot\left[Q_{\tau_{i+2}}\left(x_{i+1}, x_{i+2}\right)\right]\left[\bar{\partial}_{x_{i+2}} D_{1, x_{i+2}} \ldots\right] \ldots\left[\ldots x_{\infty}\left(x_{k}, y_{0}^{\prime}\right)\right] \\
\cdot\left|d x_{0}\right| \ldots\left|d x_{k}\right| d \tau_{1} \ldots d \tau_{k}
\end{aligned}
$$

and

$$
\begin{gathered}
\overline{\widetilde{F}_{k+1}}\left(\sum_{l=1}^{l=m}\left(\bar{\partial} g_{l}\right) D_{1} \otimes \cdots \otimes D_{k} \otimes f_{l}\right) \\
=\int_{C_{k+1}} \int_{X^{k+1}} \sum_{l=1}^{l=m} p_{\infty}\left(y_{0}, x_{0}\right)\left(\bar{\partial} g_{l}\right)\left(x_{0}\right) D_{1, x_{0}} Q_{\tau_{1}}\left(x_{0}, x_{1}\right) \ldots \\
= \pm \int_{C_{k+1}} \int_{X^{k+1}} \sum_{l=1}^{l=m} p_{\infty}\left(y_{0}, x_{0}\right) \bar{\partial}_{x_{0}} g_{l}\left(x_{0}\right) D_{1, x_{0}} Q_{\tau_{1}}\left(x_{0}, x_{1}\right) \ldots \\
\quad \pm \int_{C_{k+1}} \int_{X^{k+1}} \sum_{l=1}^{l=m} p_{\infty}\left(y_{0}, x_{0}\right) g_{l}\left(x_{0}\right) \bar{\partial}_{x_{0}} D_{1, x_{0}} Q_{\tau_{1}}\left(x_{0}, x_{1}\right) \ldots \\
= \pm \int_{C_{k+1}} \int_{X^{k+1}}\left[\bar{\partial}_{x_{0}}^{a} p_{\infty}\left(y_{0}, x_{0}\right)\right]\left[\sum_{l=1}^{l=m} f_{l}\left(x_{k}\right) g_{l}\left(x_{0}\right)\right]\left[D_{1, x_{0}} Q_{\tau_{1}}\left(x_{0}, x_{1}\right)\right] \ldots \\
\quad \pm \int_{C_{k+1}} \int_{X^{k+1}}\left[p_{\infty}\left(y_{0}, x_{0}\right)\right]\left[\sum_{l=1}^{l=m} f_{l}\left(x_{k}\right) g_{l}\left(x_{0}\right)\right]\left[x_{x_{0}} D_{1, x_{0}} Q_{\tau_{1}}\left(x_{0}, x_{1}\right)\right] \ldots \\
\left.\ldots\left[p_{\infty}\left(x_{k}, y_{0}^{\prime}\right)\right]\left|d x_{0}\right| \ldots\left|d x_{k}\right| d \tau_{1} \ldots d \tau_{k}\right) .
\end{gathered}
$$

For each of the summands dealt with by equations (13), (14) and (15) respectively, one can argue exactly as we did after (2).

This finally proves Proposition 3.

Proposition 4. Let $\beta=\beta_{1}+\cdots+\beta_{k}$ be a 0 -cycle in $C^{\bullet}\left(\operatorname{Diff}^{\bullet}(\mathcal{E})\right)$ with $\beta_{k} \in$ $\operatorname{Diff}^{\bullet}(\mathcal{E})^{\otimes k}$. Then

$$
I_{\mathrm{FLS}}(\beta)=I_{\mathrm{FLS}}\left(\beta_{1}\right) .
$$

Proof. Pick any $t>0$. If $f$ is as in Construction 1 (see p. 417), then the cycle $\beta$ is homotopic to the cycle $N_{f}(\beta)$. Since $I_{\mathrm{FLS}}$ is a Hochschild 0-cocycle of $\operatorname{Diff}^{\bullet}(\mathscr{E})$ ), we have

$$
I_{\mathrm{FLS}}(\beta)=I_{\mathrm{FLS}}\left(N_{f}(\beta)\right)=I_{\mathrm{FLS}}\left(N_{f}\left(\beta_{1}\right)\right)+\cdots+I_{\mathrm{FLS}}\left(N_{f}\left(\beta_{k}\right)\right) .
$$


By Proposition 4,

$$
\sum_{j=2}^{j=k}\left|I_{\mathrm{FLS}}\left(N_{f}\left(\beta_{j}\right)\right)\right| \leq C \epsilon(t)
$$

for some constant $C$ depending on $\beta$ only. Also

$$
N_{f}\left(\beta_{1}\right)=\beta_{1} \pm \sum_{l=1}^{l=m}\left(\bar{\partial} f_{l}\right) \otimes g_{l} \beta_{1} \pm \sum_{l=1}^{l=m} f_{l} \otimes\left(\bar{\partial} g_{l}\right) \beta_{1} .
$$

The proof of Proposition 4 can also be used to verify that

$$
\left|I_{\mathrm{FLS}}\left(\sum_{l=1}^{l=m}\left(\bar{\partial} f_{l}\right) \otimes g_{l} \beta_{1} \pm \sum_{l=1}^{l=m} f_{l} \otimes\left(\bar{\partial} g_{l}\right) \beta_{1}\right)\right| \leq C^{\prime} \epsilon(t)
$$

for some constant $C^{\prime}$ depending only on $\beta_{1}$. It follows that

$$
\left|I_{\mathrm{FLS}}(\beta)-I_{\mathrm{FLS}}\left(\beta_{1}\right)\right|=\mid I_{\mathrm{FLS}}\left(N_{f}(\beta)-I_{\mathrm{FLS}}\left(\beta_{1}\right) \mid \leq\left(C+C^{\prime}\right) \epsilon(t) .\right.
$$

Now $t$ may be chosen to be arbitrarily small. In this case, $\epsilon(t)$ becomes arbitrarily small as well. As $C+C^{\prime}$ depends only on $\beta$, the proposition follows.

Proposition 3 implies the following (surprising) statement.

Proposition 5. The linear functional

$$
D \mapsto \operatorname{str}\left(\Pi_{0} D \Pi_{0}\right)
$$

vanishes on 0 -chains of $\operatorname{Diff}^{\bullet}(\mathcal{E})$ that are commutators of elements in $\operatorname{Diff}^{\bullet}(\mathcal{E})$.

Proof. Let $D_{1}, D_{2} \in \operatorname{Diff}^{0}(\mathcal{E})$. This proposition is immediate from Proposition 4 and the following two facts: $I_{\mathrm{FLS}}\left(d\left(D_{1} \otimes D_{2}\right)\right)=0$ since $I_{\mathrm{FLS}}$ is a Hochschild 0-cocycle, and the component of $d\left(D_{1} \otimes D_{2}\right)$ in $\operatorname{Diff}^{\bullet}(\mathcal{E})$ is precisely $-D_{1} D_{2}+D_{2} D_{1}$.

Of course, when $D$ is the commutator of two purely holomorphic differential operators on $\mathcal{E}$, the above proposition is standard. The above proposition in general is however very counter-intuitive, and the author does not see any other way of proving it.

3.3. Extending the supertrace - II. Let $\operatorname{Diff}^{\bullet}(\mathcal{E})(U)$ denote $\Gamma\left(U, \operatorname{Diff}^{\bullet}(\mathcal{E})\right)$. Note that the differential on $C^{\bullet}\left(\operatorname{Diff}^{\bullet}(\mathscr{E})(U)\right)$ extends to a differential on the graded vector space $\bigoplus_{k} \mathscr{D i f f}^{\bullet}\left(\varepsilon^{\otimes k}\right)\left(U^{k}\right)[k-1]$. We denote the resulting complex by $\overline{C^{\bullet}\left(\operatorname{Diff}^{\bullet}(\mathcal{E})(U)\right)}$. 
Let $\widehat{\operatorname{Hoch}(\mathscr{D i f f}(\mathcal{E}))}$ denote the sheaf associated to the pre-sheaf

$$
\left.U \mapsto \overline{C^{\bullet}\left(\operatorname{Diff}^{\bullet}(\mathcal{E})(U)\right.}\right)
$$

of complexes of $\mathbb{C}$-vector spaces. Note that $\overline{\operatorname{Hoch}(\mathscr{D i f f}(\mathscr{E}))}$ is a complex whose terms are modules over the sheaf of smooth functions on $X$. It follows that

$$
\left.\left.\mathrm{H}^{\bullet}(\Gamma(X, \widehat{\operatorname{Hoch}(\mathscr{D i f f}(\mathcal{E})}))\right) \simeq \mathbb{H}^{\bullet}(X, \widehat{\operatorname{Hoch}(\mathscr{D i f f}(\mathcal{E})})\right) .
$$

The following proposition follows from Proposition 4.

Proposition 6. $I_{\mathrm{FLS}}$ extends to a linear functional on $\left.\Gamma(X, \widehat{\operatorname{Hoch}(\mathscr{D i f f}(\mathcal{E})})\right)$ that vanishes on the image of the differential d of $\Gamma(X, \widehat{\operatorname{Hoch}(\mathscr{D i f f}(\mathcal{E})}))$.

Proof. Part 1: Constructing str:

Let $\operatorname{Hoch}^{k}(\operatorname{Diff}(\mathscr{E}))$ denote the sheaf associated to the pre-sheaf

$$
U \mapsto \operatorname{Diff}^{\bullet}\left(\mathcal{E}^{\otimes k}\right)\left(U^{k}\right)[k-1]
$$

of graded $\mathbb{C}$-vector spaces. Then any 0 -cycle in $\Gamma(X, \widehat{\operatorname{Hoch}(\mathscr{D i f f}(\mathcal{E})}))$ is given by $\beta_{1}+\cdots+\beta_{k}$ where $\beta_{i} \in \Gamma\left(X, \widehat{\operatorname{Hoch}^{i}(\operatorname{Diff}(\mathcal{E}))}\right)$. Note that $\widehat{\operatorname{Hoch}^{1}(\operatorname{Diff}(\mathscr{E}))}=$ $\operatorname{Diff}^{\bullet}(\mathcal{E})$. It follows that $\beta_{1}$ is an element of $\operatorname{Diff}^{0}(\mathcal{E})$.

Let $\widehat{s t r}$ be the linear functional on $\Gamma(X, \widehat{\operatorname{Hoch}(\mathscr{D i f f}(\mathcal{E})}))$ that vanishes on $p$-chains whenever $p \neq 0$. For a 0 -chain $\beta=\beta_{1}+\cdots+\beta_{k}$ with $\beta_{i} \in \Gamma\left(X \widehat{\operatorname{Hoch}^{i}(\mathscr{D i f f}(\mathscr{E}))}\right)$, set

$$
\widehat{\operatorname{str}}(\beta)=I_{\mathrm{FLS}}\left(\beta_{1}\right) \text {. }
$$

This is a well-defined linear functional on the space of 0 -chains of $\Gamma(X, \widehat{\operatorname{Hoch}(\operatorname{Diff}(\mathcal{E}))})$.

Part 2: We now need to show that str vanishes on the image of $d$.

Claim A: The natural map from $\operatorname{Diff}^{\bullet}\left(\mathcal{E}^{\boxplus 2}\right)[1]$ to $\left.\Gamma\left(X, \widehat{\operatorname{Hoch}^{2}(\mathscr{D i f f}(\mathscr{E})}\right)\right)$ is a surjection of graded $\mathbb{C}$-vector spaces.

We postpone the proof of Claim A for the next part of the proof. Note that the differential on $C^{\bullet}\left(\operatorname{Diff}^{\bullet}(\mathcal{E})\right)$ extends to a differential $d$ on $\bigoplus_{k} \operatorname{Diff}^{\bullet}\left(\mathcal{E}^{\otimes k}\right)[k-1]$. This differential is a sum of the differentials $d_{\mathrm{H}}$ : Diff ${ }^{\bullet}\left(\mathcal{E}^{\otimes k}\right) \rightarrow \operatorname{Diff}{ }^{\bullet}\left(\mathcal{E}^{\boxplus k-1}\right)$ and $\bar{\partial}$. By Proposition 4 and Claim A, to show that str vanishes on the image of $d$, it suffices to show that the map $\operatorname{Diff}^{0}(\mathscr{E}) \rightarrow \mathbb{C}$,

$$
D \mapsto \operatorname{str}\left(\Pi_{0} D \Pi_{0}\right),
$$

vanishes on the image of $d_{\mathrm{H}}: \operatorname{Diff}^{0}\left(\mathcal{E}^{\bigotimes 2}\right) \rightarrow \operatorname{Diff}^{0}(\mathcal{E})$. 
Let Diff $\leq k, \cdot(\mathcal{E})$ denote $\Gamma\left(X, \operatorname{Dolb}^{\bullet}\left(X, \mathcal{O}_{X}\right) \otimes \operatorname{Diff}^{\leq k}(\mathcal{E})\right)$ where $\operatorname{Diff}^{\leq k}(\mathcal{E})$ denotes the sheaf of differential operators on $\mathcal{E}$ of order $\leq k$. Equip Diff $\leq k, \bullet(\mathcal{E})$ with the topology generated by the family of seminorms $\left\{\|\cdot\|_{K, s} \mid K \subset X\right.$ compact, $s \in$ $\left.\Gamma\left(K, \mathcal{E} \otimes \Omega^{\bullet}\right)\right\}$ given by

$$
\|D\|_{K, s}=\sup \{\|D(s)(x)\| \mid x \in K\} .
$$

The topology on $\operatorname{Diff}^{\bullet}(\mathscr{E})$ is the direct limit of the topologies on the Diff $\leq k, \bullet(\mathscr{E})$. The topology on $\operatorname{Diff}^{\bullet}\left(\varepsilon^{\otimes 2}\right)$ is defined analogously.

Note that $\operatorname{Diff}^{\bullet}(\mathcal{E})^{\otimes 2}$ is dense in $\operatorname{Diff}^{\bullet}\left(\varepsilon^{\otimes 2}\right)$. Furthermore, it follows that $d_{\mathrm{H}}: \operatorname{Diff}^{\bullet}\left(\mathcal{E}^{\bigotimes 2}\right) \rightarrow \operatorname{Diff}^{\bullet}(\mathcal{E})$ is continuous. The restriction of $d_{\mathrm{H}}$ to $\operatorname{Diff}^{0}(\varepsilon)^{\otimes 2}$ is just the map

$$
D_{1} \otimes D_{2} \mapsto D_{2} D_{1}-D_{1} D_{2} .
$$

The proposition now follows from Proposition 5.

Part 3: Proof of Claim A.

Given any element $\alpha$ of $\Gamma\left(X, \widehat{\operatorname{Hoch}^{2}(\operatorname{Diff}(\mathcal{E}))}\right)$, pick a finite cover $X=\bigcup_{i} U_{i}$ of open sets such that $\left.\alpha\right|_{U_{i}}$ is an element of $\operatorname{Diff}^{\bullet}\left(\mathcal{E}^{\unrhd 2}\right)\left(U_{i} \times U_{i}\right)[1]$. Pick a partition of unity $\left\{f_{i}\right\}$ by compactly supported smooth functions subordinate to he cover $\bigcup_{i} U_{i}$. Let $g_{i}$ be a compactly supported smooth function supported on a subset of $U_{i}$ that is identically 1 on the support of $f_{i}$. Then

$$
\left.\sum_{i}\left(f_{i} \otimes g_{i}\right) \alpha\right|_{U_{i}}
$$

is an element of $\operatorname{Diff}^{\bullet}\left(\mathcal{E}^{\bigotimes 2}\right)[1]$ whose image in $\left.\Gamma\left(X, \widehat{\operatorname{Hoch}^{2}(\mathscr{D i f f}(\mathscr{E})}\right)\right)$ is $\alpha$.

Next we note that we have a natural (degree preserving) map

$$
\gamma: \widehat{\operatorname{Hoch}}(\operatorname{Diff}(\mathcal{E})) \rightarrow \widehat{\operatorname{Hoch}(\operatorname{Diff}(\mathcal{E}))}
$$

of complexes of sheaves of $\mathbb{C}$-vector spaces on $X$. This induces a map

$$
\left.\gamma_{*}: \mathbb{Q}^{\bullet}(X, \widehat{\operatorname{Hoch}}(\operatorname{Diff}(\mathscr{E}))) \rightarrow \mathbb{Q}^{\bullet}(X, \widehat{\operatorname{Hoch}(\mathscr{D i f f}(\mathscr{E})})\right) .
$$

Recall that $\left.\mathrm{H}^{0}(\Gamma(X, \widehat{\operatorname{Hoch}(\mathscr{D i f f}(\mathcal{E})}))=\mathbb{T}^{0}(X, \widehat{\operatorname{Hoch}(\mathscr{D i f f}(\mathcal{E})})\right)$. The following corollary of Proposition 6 is immediate.

Corollary 3. The Hochschild cocycle str: $\mathrm{HH}_{0}\left(\mathrm{Diff}^{\bullet}(\mathcal{E})\right) \rightarrow \mathbb{C}$ extends to a $\mathbb{C}$ -

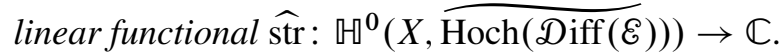

It is obvious that $\widehat{\operatorname{str}} \circ \gamma_{*}$ yields a linear functional on $\mathbb{M}^{0}(X, \widehat{\operatorname{Hoch}}(\mathscr{D i f f}(\mathcal{E})))$. We state this as a corollary for emphasis. 
Corollary 4. The linear functional $\widehat{\mathrm{str}}$ in the previous corollary gives us a linear functional $\widehat{\operatorname{tr}}: \mathbb{\boxplus}^{0}(X, \widehat{\operatorname{Hoch}}(\operatorname{Diff}(\mathcal{E}))) \rightarrow \mathbb{C}$.

We denote $\mathbb{H}^{-i}(X, \widehat{\operatorname{Hoch}}(\mathscr{D i f f}(\mathcal{E})))$ by $\widehat{\mathrm{HH}}_{-i}(\operatorname{Diff}(\mathcal{E}))$.

Now consider a global holomorphic differential operator $D$ on $\mathcal{E}$. $D$ can be thought of as an element of $\Gamma\left(X, \operatorname{Diff}^{\bullet}(\mathcal{E})\right)$ as well. Thus, $D$ gives us a 0 -cocycle of the cochain complex $\Gamma(X, \widehat{\operatorname{Hoch}(\mathscr{D i f f}(\mathcal{E}))})$. Let $\bar{D}$ denote this cocycle.

One the other hand, $D$ can be thought of as a global section of the degree 0 term of the complex $\widehat{\operatorname{Hoch}}(\mathscr{D i f f}(\mathcal{E}))$. It follows that $D$ yields a 0 -cycle in the bi-complex computing $\mathbb{H}^{\bullet}(X, \widehat{\operatorname{Hoch}}(\mathscr{D i f f}(\mathcal{E})))$. Call this 0 -cycle $\widetilde{D}$. It is easy to check that $\gamma(\widetilde{D})=\bar{D}$. We have thus proven the following proposition.

Proposition 7. The following diagram commutes:

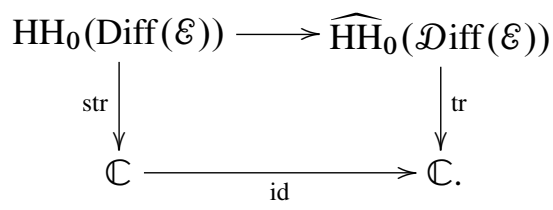

3.4. The first bookkeeping lemma. Consider $\mathcal{E}$ as a sub bundle of $\mathscr{E} \oplus \mathcal{F}$ where $\mathcal{F}$ is another vector bundle on $X$. We then have a map $\iota: \operatorname{Diff}(\mathcal{E}) \rightarrow \operatorname{Diff}(\varepsilon \oplus$ $\mathcal{F})$ of sheaves of $\mathbb{C}$-vector spaces whose restriction to $U, \iota: \Gamma(U, \mathscr{D i f f}(\mathcal{E})) \rightarrow$ $\Gamma(U, \mathcal{D i f f}(\mathcal{E} \oplus \mathcal{F}))$, is an injection that preserves addition and multiplication for each open $U \subset X$. This also induces an map $\bar{\imath}: \operatorname{Diff}^{\bullet}(\mathcal{E}) \rightarrow \operatorname{Diff}^{\bullet}(\mathcal{E} \oplus \mathscr{F})$ of sheaves of $\mathbb{C}$-vector spaces whose restriction to $U, \bar{\imath}: \operatorname{Diff}^{\bullet}(\mathscr{E})(U) \rightarrow \operatorname{Diff}^{\bullet}(\mathscr{E} \oplus \mathscr{F})(U)$, is an injection for each open $U \subset X$.

The map $\bar{\iota}$ preserves addition and multiplication though it does not preserve the identity. Thus $\bar{\imath}$ induces a map from $\widehat{\operatorname{Hoch}(\mathscr{D i f f}(\mathcal{E}))}$ to $\widehat{\operatorname{Hoch}(\mathscr{D i f f}(\mathcal{E} \oplus \mathscr{F}))}$, which we will denote by $\tilde{\imath}$. Let $\tilde{\iota}_{*}$ denote the map from $\mathrm{H}^{*}(\Gamma(X, \widehat{\operatorname{Hoch}(\mathscr{D i f f}(\mathscr{E})))})$ to $\left.\left.\mathrm{H}^{*}(\Gamma(X, \widehat{\operatorname{Hoch}(\mathscr{D i f f}(\mathcal{E} \oplus \mathcal{F}}))\right)\right)$ induced by $\tilde{\iota}$. The following "bookkeeping lemma" holds.

Lemma 1. The following diagram commutes:

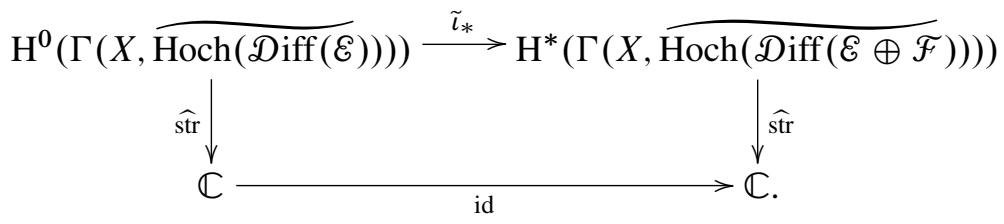

Proof. By Proposition 5 and the proof of Proposition 6, it is enough to show that

$$
\operatorname{str}\left(\Pi_{K_{0 \mathscr{E}}^{\bullet}} D \mathcal{I}_{K_{0 \mathscr{E}}^{\bullet}}\right)=\operatorname{str}\left(\Pi_{K_{0 \mathscr{E}}^{\bullet}} \tilde{\mathcal{F}}(D) \mathcal{I}_{K_{0 \mathscr{\Theta}}^{\bullet}}\right)
$$


for any $D \in \operatorname{Diff}^{0}(\mathscr{E})$.

The above equality holds since the following diagrams commute:
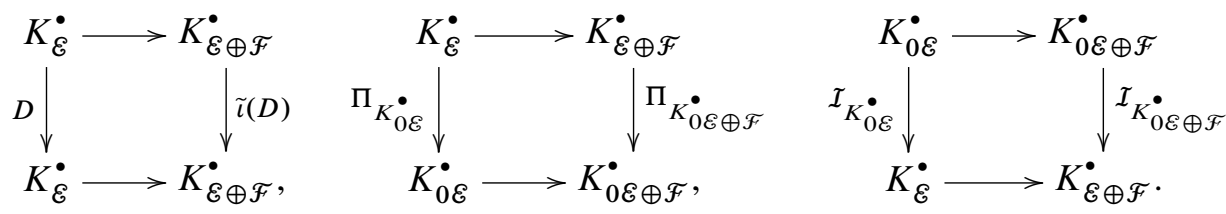

The map $\iota$ induces a map $\hat{\imath}: \widehat{\operatorname{Hoch}}(\operatorname{Diff}(\mathcal{E})) \rightarrow \widehat{\operatorname{Hoch}}(\operatorname{Diff}(\mathcal{E} \oplus \mathscr{F}))$. Let $\hat{\iota}_{*}$ denote the map induced by $\hat{\imath}$ from $\widehat{\mathrm{HH}}_{*}(\operatorname{Diff}(\mathscr{E}))$ to $\widehat{\mathrm{HH}}_{*}(\operatorname{Diff}(\mathscr{E} \oplus \mathscr{F}))$. The following corollary holds.

Corollary 5. The following diagram commutes:

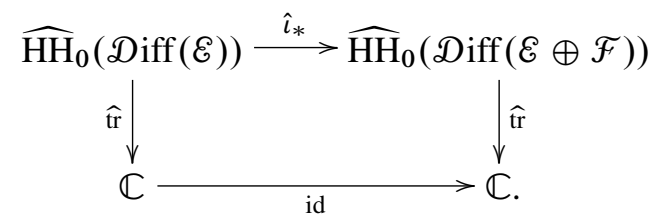

Proof. For this we only need to recall that by the definition of $\iota$ at the beginning of this section the following diagram commutes:

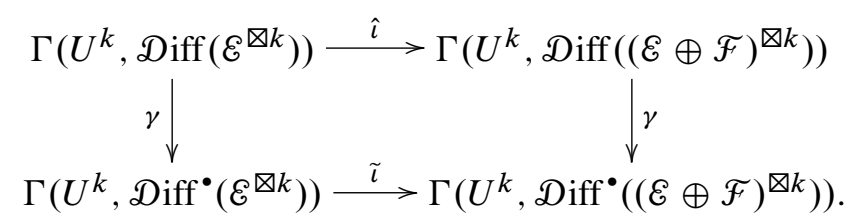

Taking hypercohomology we see that $\gamma_{*} \circ \hat{\iota}_{*}=\tilde{\iota}_{*} \circ \gamma_{*}$. The corollary now follows immediately from Lemma 1 .

\section{The completed Hochschild homology of $\mathscr{D i f f}(\mathcal{E})$ and the cohomology of $X$}

4.1. Preliminaries. Recall the definition of $\widehat{\operatorname{Hoch}}(\mathscr{D i f f}(\mathscr{E}))$ from Section 3.2. Let $\widehat{\text { Hoch }}(\operatorname{Diff}(X))$ denote $\widehat{\operatorname{Hoch}}\left(\operatorname{Diff}\left(\mathcal{O}_{X}\right)\right)$. Let $\mathbb{C}$ denote the constant sheaf on $X$ such that $\Gamma(U, \mathbb{C}) \simeq \mathbb{C}$ for every open $U \subset X$ and whose restriction maps are all identity.

Lemma 2 ([Bryl]). $\widehat{\operatorname{Hoch}}(\operatorname{Diff}(X))$ is quasi-isomorphic to $\underline{\mathbb{C}}[2 n]$ where $n$ is the dimension of $X$. 
Sketch of the proof recalled from [Bryl]. It is enough to check the above fact for any open ball $U \subset X$ at the level of pre-sheaves. In other words, it is enough to show that the complex $\operatorname{Diff}\left(U^{\bullet}\right)$ with Hochschild differential is quasi-isomorphic to $\mathbb{C}[2 n]$ as complexes of $\mathbb{C}$-vector spaces.

We filter $\operatorname{Diff}(U)$ by degree. More precisely, set $F^{-n}(\operatorname{Diff}(U)):=\operatorname{Diff}^{\leq n}(U)$. The associated graded $\operatorname{gr}(\operatorname{Diff}(U))$ is the space of functions $\mathcal{O}_{T^{*} U}$ on the cotangent bundle $T^{*} U$ that are algebraic(polynomial) along the fibres.

This filtration of $\operatorname{Diff}(U)$ is exhaustive. It yields a filtration of the complex $\operatorname{Diff}\left(U^{\bullet}\right)$ equipped with Hochschild differential. This filtration yields a spectral sequence converging to the cohomology of the complex $\operatorname{Diff}\left(U^{\bullet}\right)$. The $E_{1}^{m}:=$ $\bigoplus_{p+q=m} E_{1}^{p, q}$-term is $m$-th cohomology of the completed Hochschild complex of $\mathcal{O}_{T^{*} U}$. This is isomorphic to the space of $-m$-holomorphic forms on $T^{*} U$ that are algebraic along the fibres (see [Bryl]). Given local coordinates $z_{1}, \ldots z_{n}$ on $U$ and $y_{1}, \ldots, y_{n}$ on the fibre of $T^{*} U$, putting the weight of $d z_{i}$ equal to 0 and that of $d y_{i}$ equal to 1 for all $i$ enables us to define the notion of the weight of a holomorphic form on $T^{*} U$. In the spectral sequence of this proof-sketch, $E_{1}^{p, m-p}$ is simply the space of $-m$-forms on $T^{*} U$ of weight $-p$. Therefore, $E_{1}^{-n,-n}$ is the only non-zero summand of $E_{1}^{-2 n}$. By Theorem 3.1.1 and Corollary 2.2.2 of [Bryl], the $E_{2}^{m}=E_{\infty}^{m}$-term of this spectral sequence is the $2 n+m$-th de Rham cohomology of $T^{*} U$. This is 0 if $m \neq-2 n$ and $\mathbb{C}$ otherwise. This proves the lemma. Moreover, $E_{2}^{-n,-n}$ is the only nontrivial summand of $E_{2}^{-2 n}$. Therefore, the $-2 n$-th cohomology of this complex can be identified with $E_{2}^{-n,-n}$.

Lemma 3. $\widehat{\text { Hoch }}(\operatorname{Diff}(\mathcal{E}))$ is quasi-isomorphic to $\underline{\mathbb{C}}[2 n]$ where $n$ is the dimension of $X$.

Proof. This is again something that needs to be verified locally. We imitate the proof of the Morita invariance of Hochschild homology in [Lod], Section 1.2, here.

Part 1: Recalling the proof of Morita invariance of the Hochschild homology of a $\mathbb{C}$-algebra.

Recall from [Lod] that if $A$ is any $\mathbb{C}$-algebra, and if $\operatorname{Mat}_{r}(A)$ denotes the algebra of $r \times r$ matrices with entries in $A$, then we have a map tr from the Hochschild complex of $\operatorname{Mat}_{r}(A)$ to that of $A$. The Hochschild chain $M_{1} \otimes \cdots \otimes M_{k}$ is mapped to $\operatorname{tr}\left(M_{1} \odot \cdots \odot M_{k}\right)$ where $\odot: \operatorname{Mat}_{r}(A) \otimes \operatorname{Mat}_{r}(B) \rightarrow \operatorname{Mat}_{r}(A \otimes B)$ is an exterior multiplication. There is also a map inc of complexes into the opposite direction which is induced by the inclusion of $A$ in $\operatorname{Mat}_{r}(A)$ taking an element $a$ of $A$ to the matrix with $a \cdot E_{11}$. Then $\operatorname{tr} \circ$ inc $=$ id and there is a presimplicial homotopy $h$ from inc $\circ \operatorname{tr}$ to id. This homotopy is given by $h=\sum_{i}(-1)^{i} h_{i}$ where $h_{i}: \operatorname{Mat}_{r}(A)^{\otimes k+1} \rightarrow$ 
$\operatorname{Mat}_{r}(A)^{\otimes k+2}$ is defined by the formula

$$
\begin{aligned}
& h_{i}\left(\alpha^{0} \otimes \cdots \otimes \alpha^{k}\right) \\
& \quad=\sum E_{j 1}\left(\alpha_{j r}^{0}\right) \otimes E_{11}\left(\alpha_{r m}^{1}\right) \otimes \cdots \otimes E_{11}\left(\alpha_{p q}^{i}\right) \otimes E_{1 q}(1) \otimes \alpha^{i+1} \otimes \cdots \otimes \alpha^{k} .
\end{aligned}
$$

The sum here is over all possible tuples of indices $(j, r, \ldots, p, q)$, see [Lod], Section 1.2 , and $E_{i j}(x)=x \cdot E_{i j}$, where $E_{i j}$ is the elementary matrix whose only non-zero entry is a 1 at the $i j$ position.

Let $U$ be an open ball contained in $X$.

Part 2: Morita invariance of the completed Hochschild homology of Diff $(U)$.

Recall that the completed Hochschild complex $\left.\overline{C^{\bullet}(\operatorname{Diff}(U)}\right)$ is obtained by equipping the graded vector space $\bigoplus_{k \geq 1} \operatorname{Diff}\left(U^{k}\right)[k-1]$ with the Hochschild differential. In this complex, $\operatorname{Diff}\left(U^{k}\right)$ should be viewed as the $k$-th completed tensor power of $\operatorname{Diff}(U)$. With this in mind, the $k$-th completed tensor power of $\operatorname{Mat}_{r}(\operatorname{Diff}(U))$ will be Mat $r(\mathbb{C})^{\otimes k} \otimes \operatorname{Diff}\left(U^{k}\right)$. One may verify that the Hochschild differential on $C^{\bullet}\left(\operatorname{Mat}_{r}(\operatorname{Diff}(U))\right)$ extends to a differential on the graded vector space $\bigoplus_{k \geq 1} \operatorname{Mat}_{r}(\mathbb{C})^{\otimes k} \otimes \operatorname{Diff}\left(U^{k}\right)[k-1]$. The resulting complex is the completed Hochschild complex $\left.\overline{C^{\bullet}\left(\operatorname{Mat}_{r}(\operatorname{Diff}(U))\right.}\right)$.

One can verify without much difficulty that tr and inc extend to maps of complexes

$$
\left.\operatorname{tr}: \overline{C^{\bullet}\left(\operatorname{Mat}_{r}(\operatorname{Diff}(U))\right.}\right) \rightarrow \overline{C^{\bullet}(\operatorname{Diff}(U))}
$$

and

$$
\text { inc } \left.: \overline{C^{\bullet}(\operatorname{Diff}(U))} \rightarrow \overline{C^{\bullet}\left(\mathrm{Mat}_{r}(\operatorname{Diff}(U))\right.}\right)
$$

respectively such that $\operatorname{tr} \circ \mathrm{inc}=\mathrm{id}$. It is also useful for us to note explicitly that if $m_{1}, \ldots, m_{k} \in \operatorname{Mat}_{r}(\mathbb{C})$ and $\alpha \in \operatorname{Diff}\left(U^{k}\right)$, then

$$
\operatorname{tr}\left(m_{1} \otimes \cdots \otimes m_{k} \otimes \alpha\right)=\operatorname{tr}\left(m_{1} \circ \cdots \circ m_{k}\right) \alpha .
$$

Moreover $h$ extends to a map $\left.\left.h: \overline{C^{k}\left(\operatorname{Mat}_{r}(\operatorname{Diff}(U))\right.}\right) \rightarrow \overline{C^{k+1}\left(\operatorname{Mat}_{r}(\operatorname{Diff}(U))\right.}\right)$ for all $k$ with $d h+h d=\mathrm{id}-$ inc $\circ \operatorname{tr}$. Thus tr: $\left.\left.\overline{C^{\bullet}\left(\operatorname{Mat}_{r}(\operatorname{Diff}(U))\right.}\right) \rightarrow \overline{C^{\bullet}(\operatorname{Diff}(U)}\right)$ is a quasi-isomorphism. It follows from Lemma 2 that $\left.\overline{C^{\bullet}\left(\operatorname{Mat}_{r}(\operatorname{Diff}(U))\right.}\right)$ is quasiisomorphic to $\mathbb{C}[2 n]$.

Part 3: Proof of the lemma.

Let $r$ be the rank of $\varepsilon$. If $U$ is an open ball of $X$, on which $\varepsilon$ is trivial, then $\operatorname{Diff}\left(\left.\mathscr{E}\right|_{U}\right)$ is isomorphic to $\operatorname{Mat}_{r}(\operatorname{Diff}(U))$ as topological algebras. The actual isomorphism depends on the choice of (holomorphic) trivialization of $\mathcal{E}$. For a holomorphic trivialization $\phi$ of $\mathcal{E}$ over $U$, let $\phi_{\circ}$ denote the isomorphism between $\operatorname{Diff}\left(\left.\mathscr{E}\right|_{U}\right)$ and $\operatorname{Mat}_{r}(\operatorname{Diff}(U))$. Then $\phi_{\circ}$ induces an isomorphism of complexes $C^{\bullet}(\mathscr{D i f f}(\mathscr{E})(U)) \rightarrow C^{\bullet}\left(\operatorname{Mat}_{r}(\operatorname{Diff}(U))\right)$. This extends to an isomorphism 
of complexes $\left.\phi_{*}: \overline{C^{\bullet}(\mathscr{D i f f}(\mathcal{E})(U))} \rightarrow \overline{C^{\bullet}\left(\operatorname{Mat}_{r}(\operatorname{Diff}(U))\right.}\right)$. We know however that $\left.\overline{C^{\bullet}\left(\operatorname{Mat}_{r}(\operatorname{Diff}(U))\right.}\right)$ is quasi-isomorphic to $\mathbb{C}[2 n]$.

The lemma will follow provided that we check that the quasi-isomorphism $\operatorname{tr} \circ \phi_{*}$ is independent of the choice of trivialization $\phi$. We will show that if $\psi$ is another holomorphic trivialization of $\mathcal{E}$ over $U$, then the maps $\operatorname{tr} \circ \psi_{*}=\operatorname{tr} \circ \phi_{*}$ of complexes from $\left.\overline{C^{\bullet}\left(\operatorname{Diff}\left(\left.\mathcal{E}\right|_{U}\right)\right.}\right)$ to $\overline{C^{\bullet}(\operatorname{Diff}(U))}$ induce the same map on cohomology.

Note that $\left.\left.\phi_{*} \circ \psi_{*}^{-1}: \overline{C^{\bullet}\left(\operatorname{Mat}_{r}(\operatorname{Diff}(U))\right.}\right) \rightarrow \overline{C^{\bullet}\left(\operatorname{Mat}_{r}(\operatorname{Diff}(U))\right.}\right)$ is induced by the map $\operatorname{Mat}_{r}(\operatorname{Diff}(U)) \rightarrow \operatorname{Mat}_{r}(\operatorname{Diff}(U))$ taking an element of $\operatorname{Mat}_{r}(\operatorname{Diff}(U))$ to its conjugate by an $(r \times r)$-matrix $N$ of holomorphic functions on $U$. Denote this map of completed Hochschild complexes by $c_{N}$. We only need to show that the maps $\operatorname{tr} \circ c_{N}$ and tr of complexes of $\mathbb{C}$-vector spaces induce the same map on cohomology.

Recall that $\operatorname{Diff}(U)$ is a filtered algebra with $F_{-m}(\operatorname{Diff}(U))=\operatorname{Diff} \leq m(U)$, the space of (holomorphic) differential operators on $U$ of order at most $m$. This yields a filtration on $\operatorname{Mat}_{r}(\operatorname{Diff}(U))$ with $F_{-m}\left(\operatorname{Mat}_{r}(\operatorname{Diff}(U))\right)=\operatorname{Mat}_{r}\left(\operatorname{Diff}^{\leq m}(U)\right)$. We

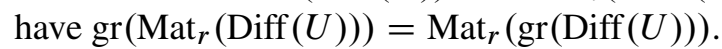

This filtration gives us a spectral sequence with $E_{1}^{n}=\widehat{\mathrm{HH}}_{-n}\left(\operatorname{gr}\left(\operatorname{Mat}_{r}(\operatorname{Diff}(U))\right)\right)$ and $E_{\infty}^{n}=\widehat{\mathrm{HH}}_{-n}\left(\operatorname{Mat}_{r}(\operatorname{Diff}(U))\right)$. The endomorphism induced by the endomorphism $\alpha \leadsto N \alpha N^{-1}$, where $N$ is a matrix of holomorphic functions, preserves the filtration on $\operatorname{Mat}_{r}(\operatorname{Diff}(U))$ and thus induces an endomorphism of a spectral sequence on the spectral sequence described above.

Note that $\operatorname{gr}\left(\operatorname{Mat}_{r}(\operatorname{Diff}(U))\right)=\operatorname{Mat}_{r}(\operatorname{gr}(\operatorname{Diff}(U)))$ and that the endomorphism induced by conjugation by $N$ is still conjugation by $N$. Denote this endomorphism by $c_{N}$ as well. We now claim that the following diagram commutes up to cohomology:

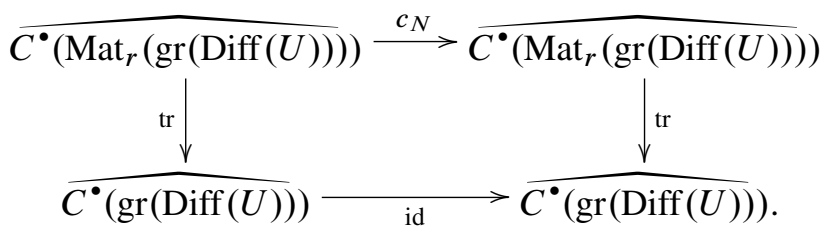

To see this, note that if $\left.\alpha \in \overline{C^{k}(\operatorname{gr}(\operatorname{Diff}(U))}\right)$ is a cocycle, then it follows that $\alpha=\operatorname{tr}\left(\frac{1}{r} \mathrm{id} \otimes \cdots \otimes \mathrm{id} \otimes \alpha\right)$. In other words, $\alpha$ is the trace of a "scalar" matrix whose diagonal elements are $\alpha$ up to a scalar factor. Now

$$
\begin{aligned}
c_{N}(\mathrm{id} \otimes \cdots \otimes \mathrm{id} \otimes \alpha) & =(N \otimes \cdots \otimes N)(\mathrm{id} \otimes \cdots \otimes \mathrm{id} \otimes \alpha)\left(N^{-1} \otimes \cdots \otimes N^{-1}\right) \\
& =(\mathrm{id} \otimes \cdots \otimes \mathrm{id} \otimes \alpha),
\end{aligned}
$$

so $\left(\operatorname{tr} \circ c_{N}\right)_{*}(\overline{\mathrm{id} \otimes \cdots \otimes \mathrm{id} \otimes \alpha})=\operatorname{tr}_{*}(\overline{\mathrm{id} \otimes \cdots \otimes \mathrm{id} \otimes \alpha})$ where $\overline{\mathrm{id} \otimes \cdots \otimes \mathrm{id} \otimes \alpha}$ denotes the class in cohomology of the cocycle $\overline{\mathrm{id} \otimes \cdots \otimes \text { id } \otimes \alpha}$.

Therefore, the map of spectral sequences induced by $\operatorname{tr} \circ c_{N}$ coincides with that induced by $\operatorname{tr}$ at the $E_{1}$-level, and hence at the $E_{2}=E_{\infty}$-level. Finally, by part 2 of 
this proof and by the proof-sketch for Lemma $2, E_{2}^{-n,-n}$ is the only non-zero $E_{2}$-term in this spectral sequence, and $E_{2}^{-n,-n} \simeq \mathbb{C}$. It follows that the endomorphisms on cohomology induced by $\operatorname{tr}$ and $\operatorname{tr} \circ c_{N}$ are indeed the endomorphisms they induce on $E_{2}^{-n,-n}$. We have just shown that the maps induced tr and $\operatorname{tr} \circ c_{N}$ on $E_{r}$-terms coincide for any $r \geq 1$. This proves the lemma.

We can now state the following immediate corollary to Lemma 3.

Corollary 6. $\widehat{\mathrm{HH}}_{-i}(\operatorname{Diff}(\mathcal{E})) \simeq \mathrm{H}^{2 n-i}(X, \mathbb{C})$.

We denote the isomorphism described in this corollary by $\beta_{\mathcal{E}}$.

4.2. The second "bookkeeping" lemma. We once more look at the situation where $\mathcal{E}$ is a direct summand of $\mathcal{E} \oplus \mathcal{F}$. Notation is as in Section 3.4 of this article. We have a map $\iota: \operatorname{Diff}(\mathscr{E}) \rightarrow \operatorname{Diff}(\mathcal{E} \oplus \mathcal{F})$. This induces a map, denoted by $\hat{\iota}$, from $\widehat{\operatorname{Hoch}}(\operatorname{Diff}(\mathscr{E}))$ to $\widehat{\operatorname{Hoch}}(\operatorname{Diff}(\mathcal{E} \oplus \mathscr{F}))$. The following lemma holds.

Lemma 4. The following diagram commutes:

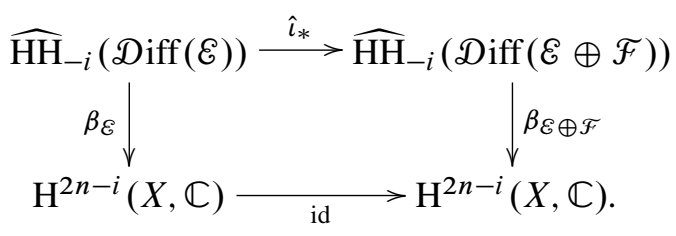

Proof. Step 1: Let $\mathrm{D}\left(\mathrm{Sh}_{\mathbb{C}}(X)\right)$ denote the derived category of sheaves of $\mathbb{C}$-vector spaces on $X$.

Recall that in the proof of Lemma 3 we showed that the complex $\widehat{\text { Hoch }}(\mathscr{D i f f}(\mathcal{E}))$ of sheaves of $\mathbb{C}$-vector spaces is quasi-isomorphic to $\widehat{\operatorname{Hoch}}\left(\mathscr{D} \operatorname{iff}\left(\mathcal{O}_{X}\right)\right)$. Denote this quasi-isomorphism by $i_{\mathcal{E}}$. Now $\widehat{\operatorname{Hoch}}\left(\operatorname{Diff}\left(\mathcal{O}_{X}\right)\right)$ is quasi-isomorphic to $\underline{\mathbb{C}}[2 n]$. Let $i$ denote this quasi-isomorphism for this proof.

It suffices to verify that the following diagram commutes in $\mathrm{D}\left(\mathrm{Sh}_{\mathbb{C}}(X)\right)$ :

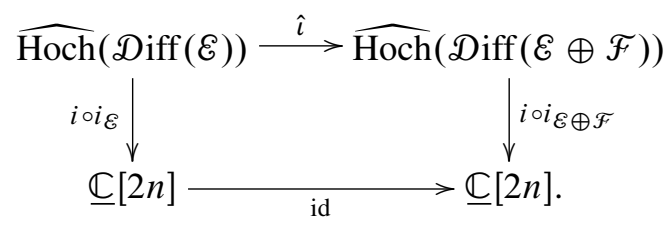

Since a sheaf of $\mathbb{C}$-vector spaces is injective iff it is flabby (see [Riet], Lemma 3.3), the constant sheaf $\mathbb{C}$ is an injective object in the category of sheaves of $\mathbb{C}$-vector spaces on $X$. It follows from this that

$$
\operatorname{Hom}_{\mathrm{D}\left(\mathrm{Sh}_{\mathbb{C}}(X)\right)}(\underline{\mathbb{C}}, \underline{\mathbb{C}}) \simeq \mathbb{C} .
$$


The diagram (16) thus commutes in $\mathrm{D}\left(\mathrm{Sh}_{\mathbb{C}}(X)\right)$ up to a scalar factor. Checking that that scalar factor is 1 can be "done locally". It therefore suffices to verify that there exists a neighbourhood $U$ of every point in $X$ such that the following diagram commutes in $\mathrm{D}\left(\mathrm{Sh}_{\mathbb{C}}(U)\right)$ :

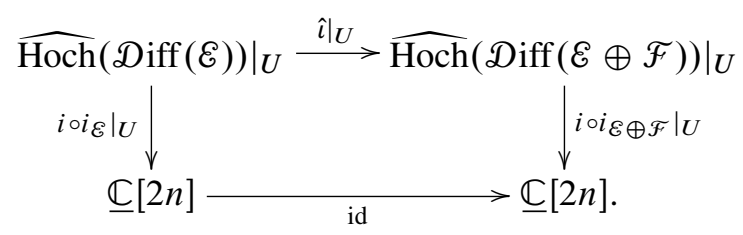

Step 2: Verifying (17).

It suffices to verify (17) at the level of pre-sheaves. We must therefore prove that the following diagram commutes in the category of complexes of $\mathbb{C}$-vector spaces up to cohomology:

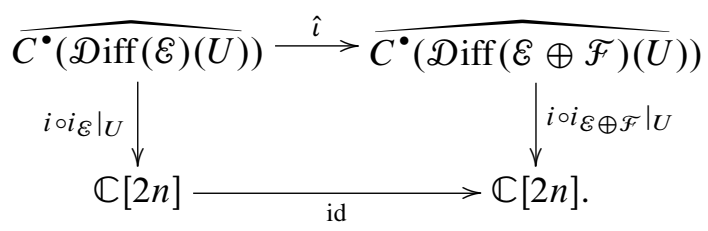

To verify that the above diagram commutes up to cohomology, it suffices to verify that the diagram below commutes up to cohomology in the category of complexes of $\mathbb{C}$-vector spaces:

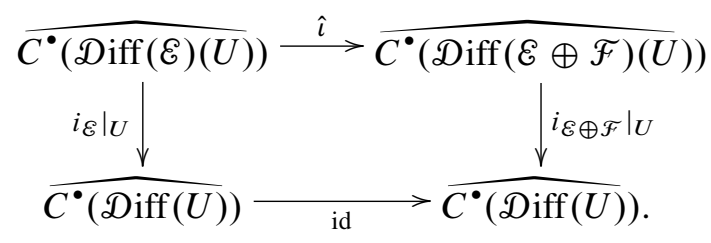

Most of the hard work necessary for this step has already been done. Let $\phi_{\mathcal{E}}$ and $\phi_{\mathcal{F}}$ denote holomorphic trivializations over $U$ of $\mathcal{E}$ and $\mathcal{F}$, respectively. Then $\phi:=\phi_{\mathcal{E}} \oplus \phi_{\mathcal{F}}$ is a holomorphic trivialization of $\mathcal{E} \oplus \mathscr{F}$ over $U$.

This yields the following commutative diagram, all of whose morphisms are continuous. Here $r$ and $s$ denote the ranks of $\mathcal{E}$ and $\mathscr{F}$, respectively:

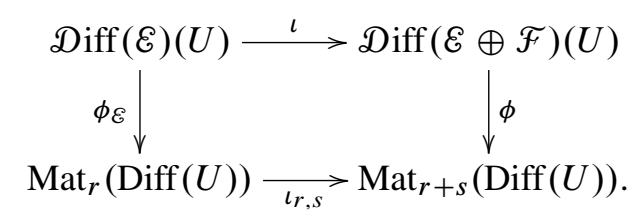


This gives the following commutative diagram:

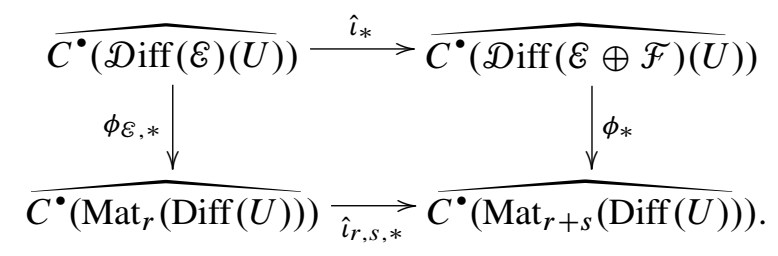

Here $\iota_{r, s}$ denotes the embedding from $\operatorname{Mat}_{r}(\operatorname{Diff}(U))$ to $\operatorname{Mat}_{r+s}(\operatorname{Diff}(U))$ which takes a matrix $\alpha \in \operatorname{Mat}_{r}(\operatorname{Diff}(U))$ to $\bar{\alpha} \in \operatorname{Mat}_{r+s}(\operatorname{Diff}(U))$, where $\bar{\alpha}_{i j}=\alpha_{i j}$ if $(i, j) \in\{1, \ldots, r\} \times\{1, \ldots, r\}$ and $\bar{\alpha}_{i j}=0$ otherwise.

Now we showed that $\left.i_{\mathcal{E}}\right|_{U}=\operatorname{tr}_{r} \circ \phi_{\mathcal{E}, *}$ in part 3 of the proof Lemma 3. Here $\operatorname{tr}_{r}$ is the map tr: $\left.\overline{C^{\bullet}\left(\operatorname{Mat}_{r}(\operatorname{Diff}(U))\right.}\right) \rightarrow \overline{C^{\bullet}(\operatorname{Diff}(U))}$ described in the proof of Lemma 3. Similarly, $i_{\left.(\mathscr{E} \oplus \mathcal{F})\right|_{U}}=\operatorname{tr}_{r+s} \circ \phi_{*}$.

It therefore suffices to prove that the following diagram commutes:

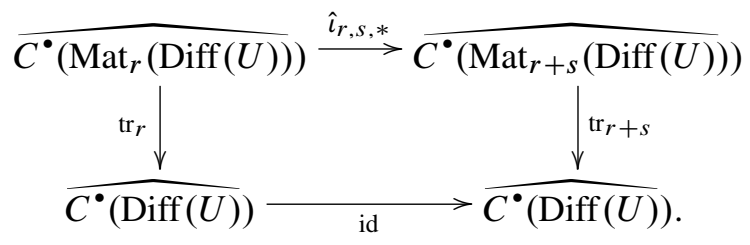

This follows immediately from the explicit formula for $\operatorname{tr}_{r}$ recalled in part 2 of the proof of Lemma 3. This finally verifies (18), thus proving the lemma.

\subsection{Proof of Theorem 2}

Proof of Theorem 2. Corollary 5 states that the following diagram commutes:

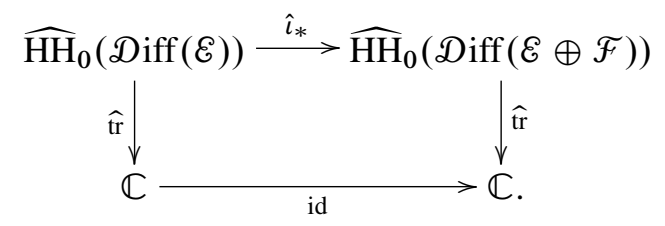

Lemma 4 for $i=0$ now states that the following diagram commutes:

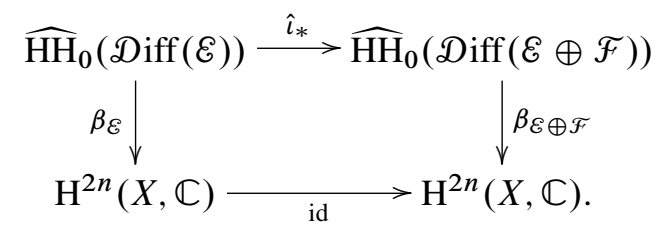


It follows that $\hat{\mathrm{tr}} \circ \beta_{\mathcal{E}}{ }^{-1}=\widehat{\mathrm{tr}} \circ \beta_{\mathcal{E} \oplus \mathcal{F}^{-1}}$. In the notation of Theorem 2, this says that $I_{\mathcal{E}}=I_{\mathcal{E} \oplus \mathscr{F}}$. A symmetric argument shows that $I_{\mathscr{F}}=I_{\mathcal{E} \oplus \mathcal{F}}$. This completes the proof of Theorem 2 .

\section{The completed cyclic homology of $\operatorname{Diff}(\mathscr{E})$}

\subsection{Recollections on cyclic homology}

5.1.1. Tsygan's double complex. Let $\mathcal{A}$ be a dg $\mathbb{C}$-algebra such that $\mathcal{A}^{n}=0$ for almost all $n$. Let $d_{\text {bar }}$ and $d_{\text {Hoch }}$ denote the differentials of $\operatorname{bar}^{\bullet}(\mathcal{A})$ and $C^{\bullet}(\mathcal{A})$ respectively.

For homogenous elements $a_{1}, \ldots, a_{k}$ of $\mathcal{A}$ of degrees $d_{1}, \ldots, d_{k}$, respectively, let the map $a_{1} \otimes \cdots \otimes a_{k} \leadsto(-1)^{\left(d_{k}+1\right)\left(d_{1}+\cdots+d_{k-1}+k-1\right)} a_{k} \otimes a_{1} \otimes \cdots \otimes a_{k-1}$ be denoted by $\tau: \mathscr{A}^{\otimes k} \rightarrow \mathcal{A}^{\otimes k}$. Let $N: \mathcal{A}^{\otimes k} \rightarrow \mathcal{A}^{\otimes k}$ be the map $N=1+\tau+\cdots+\tau^{k-1}$.

The maps $\tau$ and $N$ induce maps from $\mathscr{A}^{\otimes k}$ to $\mathscr{A}^{\otimes k}$ for any $k$. It follows that $\tau$ and $N$ induce maps from $\operatorname{bar}^{-n}(\mathcal{A})$ to $C^{-n}(\mathcal{A})$ and from $C^{-n}(\mathcal{A})$ to $\operatorname{bar}^{-n}(\mathcal{A})$, respectively, for any $n$.

Consider the following double complex the degree of whose non-zero columns is non-positive:

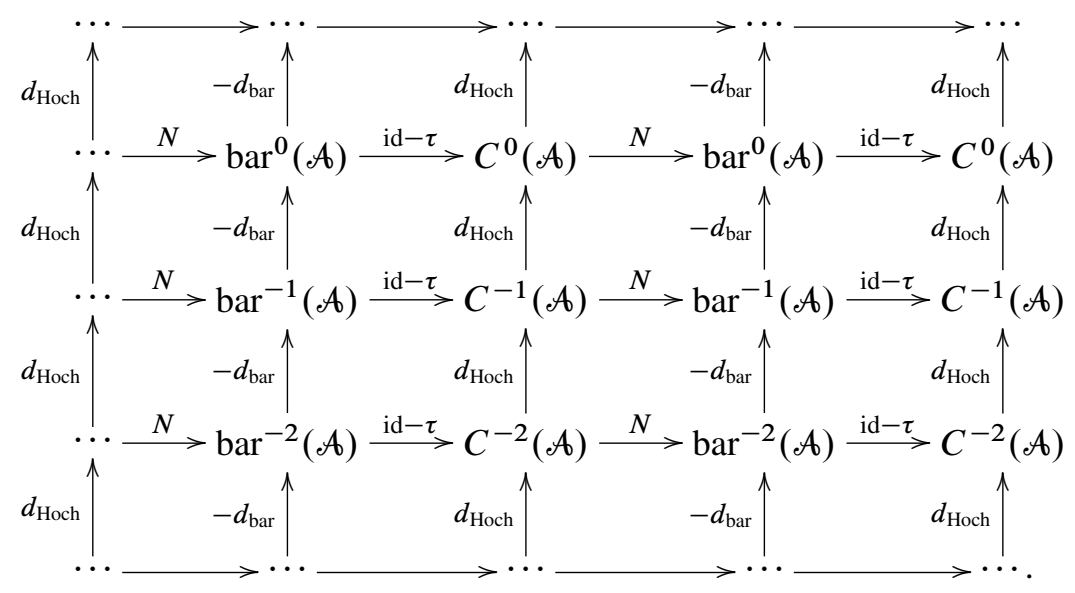

We denote the above double complex by $\mathrm{CC}^{\bullet \bullet \bullet}(\mathcal{A})$. Then $\mathrm{CC}^{p, q}(\mathcal{A})=C^{q}(\mathcal{A})$ if $p$ is even and non-positive, $\mathrm{CC}^{p, q}(\mathcal{A})=\operatorname{bar}^{q}(\mathcal{A})$ if $p$ is odd and negative, and $\mathrm{CC}^{p, q}(\mathcal{A})=0$ otherwise.

The vertical differential $d_{v}: \mathrm{CC}^{p, q}(\mathcal{A}) \rightarrow \mathrm{CC}^{p, q+1}(\mathcal{A})$ is $d_{\text {Hoch }}$ if $p$ is even and non-positive, and $-d_{\mathrm{bar}}$ if $p$ is odd and negative.

The horizontal differential $d_{h}: \mathrm{CC}^{p, q}(\mathcal{A}) \rightarrow \mathrm{CC}^{p+1, q}(\mathcal{A})$ is given by id $-\tau$ if $p$ is odd and negative and $N$ if $p$ is even and negative. 
The double complex $\mathrm{CC}^{\bullet, \bullet}(\mathcal{A})$ is called the Tsygan double complex of $\mathcal{A}$.

Definition 7. The cyclic complex of $\mathcal{A}$ is the total complex of $\mathrm{CC}^{\bullet, \bullet}(\mathcal{A})$. It is denoted by $\operatorname{Cycl}^{\bullet}(\mathcal{A})$.

Definition 8. The cyclic homology $\mathrm{HC}_{-i}(\mathcal{A})$ is the $-i$-th cohomology of $\mathrm{Cycl}^{\bullet}(\mathcal{A})$.

As in Section 2.1, contrary to the standard practice, we refer to a $-i$ cocycle of $\operatorname{Cycl}^{\circ}(\mathcal{A})$ as an $i$-cycle in $\operatorname{Cycl}^{\circ}(\mathcal{A})$. The following standard propositions are important to us.

Proposition 8. (i) If $\mathcal{B}=\operatorname{End}\left(V^{\bullet}\right)$, where $V^{\bullet}$ is a finite dimensional graded $\mathbb{C}$ vector space with zero differential, then

$$
\mathrm{HC}_{-2 i}(\mathscr{B}) \simeq \mathbb{C}
$$

for all $i \geq 0$. All other cyclic homologies of $\mathcal{B}$ vanish.

(ii) The map $\operatorname{tr}_{2 i}$ yielding the above isomorphism is obtained by mapping the class in $\mathrm{HC}_{-2 i}(\mathscr{B})$ of a $2 i$-cycle in $\mathrm{Cycl}^{\bullet}(\mathscr{B})$ given by a tuple $\left(b_{2 i}, b_{2 i-1}, \ldots, b_{0}\right)$ to $\operatorname{str}\left(b_{0}\right)$, where $b_{k} \in \mathrm{CC}^{-2 i+k,-k}(\mathscr{B})$.

(iii) The map $\operatorname{tr}_{2 i}$ yielding the above isomorphism maps the class in $\mathrm{HC}_{-2 i}(\mathscr{B})$ of a $2 i$-cycle in $\operatorname{Cycl}^{\bullet}(\mathscr{B})$ given by a tuple $\left(b_{2 i}, b_{2 i-1}, \ldots, b_{0}, b_{-1}, \ldots, b_{-2 j}\right)(j>0$, $\left.b_{k} \in \mathrm{CC}^{-2 i+k,-k}(\mathscr{B})\right)$ to $\operatorname{str}\left(b_{0}\right)$.

Proof. There is a spectral sequence, namely the one that arises out of the filtration of the double complex $\mathrm{CC}^{\bullet, \bullet}(\mathscr{B})$ by columns, converging to $\mathrm{HC} .(\mathscr{B})$ such that $E_{1}^{p, q}=$ $\mathscr{H}^{q}\left(\mathrm{CC}^{p, \bullet}(\mathscr{B})\right)$. Since $\mathscr{B}$ has a unit, bar ${ }^{\bullet}(\mathscr{B})$ is acyclic (see p. 411). Now $C^{\bullet}(\mathscr{B})$ is quasi-isomorphic to $\mathbb{C}$ concentrated in degree 0 by Proposition 1 . It follows that $E_{1}^{-2 i, 0} \simeq \mathbb{C}$ for all $i \geq 0$ and $E_{1}^{p, q}=0$ for all other $(p, q)$. This spectral sequence therefore collapses at $E_{1}$. It follows that $\mathrm{HC}_{-2 i}(\mathscr{B}) \simeq E_{1}^{-2 i, 0} \simeq \mathbb{C}$ for all $i \geq 0$. This proves part (i).

Let $F^{\bullet}$ be the filtration on $\mathrm{Cycl}^{\bullet}(\mathscr{B})$ yielding the spectral sequence in part (i). Consider a tuple $\left(b_{2 i}, b_{2 i-1}, \ldots, b_{0}\right)$ with $b_{k} \in \mathrm{CC}^{-2 i+k,-k}(\mathscr{B})$ that yields a cyclic cycle. Then $\left(b_{2 i}, b_{2 i-1}, \ldots, b_{0}\right) \in F^{-2 i} \mathrm{Cycl}^{\bullet}(\mathscr{B})$. It follows that the image of $\left(b_{2 i}, b_{2 i-1}, \ldots, b_{0}\right)$ in $\frac{F^{-2 i} \operatorname{Cycl}^{\bullet}(\mathscr{B})}{F^{-2 i+1} \operatorname{Cycl}^{\bullet}(\mathscr{B})} \simeq C^{\bullet}(\mathscr{B})$ is the Hochschild 0-cycle $b_{0}$. Note that $E_{1}^{-2 i+j,-j}=0$ for $j \neq 0$ and $E_{1}^{-2 i, 0}=\mathrm{H}^{0}\left(\frac{F^{-2 i} \mathrm{Cycl}^{\bullet}(\mathscr{B})}{F^{-2 i+1} \mathrm{Cycl}^{\bullet}(\mathscr{B})}\right) \simeq \mathrm{HH}_{0}(\mathscr{B})$. It follows that the image of the tuple $\left(b_{2 i}, b_{2 i-1}, \ldots, b_{0}\right)$ in $E_{1}^{-2 i, 0}$, and hence in $\mathrm{HC}_{-2 i}(\mathscr{B})$, is the image of $b_{0}$ in $\mathrm{HH}_{0}(\mathscr{B})$. By Proposition 1 , this is precisely $\operatorname{str}\left(b_{0}\right)$. This shows that $\operatorname{tr}_{2 i}\left(\widehat{\left(b_{2 i}, \ldots, b_{0}\right)}\right)=\operatorname{str}\left(b_{0}\right)$ where $\overline{\left(b_{2 i}, \ldots, b_{0}\right)}$ is the class in $\mathrm{HC}_{-2 i}(\mathscr{B})$ of the cycle obtained from $\left(b_{2 i}, b_{2 i-1}, \ldots, b_{0}\right)$. This proves part (ii). 
To prove part (iii), note that if $\left(b_{2 i}, b_{2 i-1}, \ldots, b_{0}, b_{-1}, \ldots, b_{-2 j}\right)(j>0)$ is a cyclic cycle, then $b_{-2 j}$ is a Hochschild $-2 j$-cycle. It follows from Proposition 1 that $b_{-2 j}=d_{\mathrm{Hoch}} c_{-2 j+1}$ for some $c_{-2 j+1} \in C^{2 j-1}(\mathscr{B})$. Now consider $c_{-2 j+1} \in \mathrm{CC}^{-2 i-2 j, 2 j-1}(\mathscr{B})$ as an element of $\mathrm{Cycl}^{-2 i-1}(\mathscr{B})$. Then the cycle $\left(b_{2 i}, b_{2 i-1}, \ldots, b_{0}, b_{-1}, \ldots, b_{-2 j}\right)-d_{\text {Cycl }} c_{-2 j+1}$ arises out of the cycle $\left(b_{2 i}, b_{2 i-1}, \ldots, b_{0}, b_{-1}, \ldots, b_{-2 j+1}+N c_{-2 j+1}, 0\right)$ of $\mathrm{CC}^{\bullet \bullet \bullet}(\mathscr{B})$. Therefore the class of $\left(b_{2 i}, b_{2 i-1}, \ldots, b_{0}, b_{-1}, \ldots, b_{-2 j}\right)$ in $\mathrm{HC}_{-2 i}(\mathscr{B})$ is the same as the class of $\left(b_{2 i}, b_{2 i-1}, \ldots, b_{0}, b_{-1}, \ldots, b_{-2 j+1}+N c_{-2 j+1}, 0\right)$ in $\mathrm{HC}_{-2 i}(\mathscr{B})$. Since the bar complex of $\mathscr{B}$ is acyclic, it follows that $b_{-2 j+1}+N c_{-2 j+1}=d_{\text {bar }} c_{-2 j+2}$ for some element $c_{-2 j+2}$ of $\operatorname{bar}^{2 j-2}(\mathscr{B})$. The previous step can be repeated to show that the class of $\left(b_{2 i}, b_{2 i-1}, \ldots, b_{0}, b_{-1}, \ldots, b_{-2 j}\right)$ in $\mathrm{HC}_{-2 i}(\mathcal{B})$ is the same as the class of $\left(b_{2 i}, b_{2 i-1}, \ldots, b_{0}, b_{-1}, \ldots, b_{-2 j+2}+(\mathrm{id}-\tau) c_{-2 j+2}, 0,0\right)$ in $\mathrm{HC}_{-2 i}(\mathscr{B})$. This process can be continued to show that the class of $\left(b_{2 i}, b_{2 i-1}, \ldots, b_{0}, b_{-1}, \ldots, b_{-2 j}\right)$ in $\mathrm{HC}_{-2 i}(\mathscr{B})$ is the same as the class of $\left(b_{2 i}, b_{2 i-1}, \ldots, b_{0}+(\mathrm{id}-\tau) c_{0}\right)$ in $\mathrm{HC}_{-2 i}(\mathscr{B})$ for some element $c_{0}$ of $\operatorname{bar}^{0}(\mathscr{B})$. By part (ii) of this proposition, this is equal to $\operatorname{str}\left(b_{0}+(\right.$ id $\left.-\tau) c_{0}\right)$. But $\operatorname{str}\left((\mathrm{id}-\tau) c_{0}\right)=0$ for any element $c_{0}$ of $\operatorname{bar}^{0}(\mathcal{B})$. This proves (iii).

If $\mathcal{A}$ is a (unital) dg $\mathbb{C}$-algebra, let $\mathrm{CC}^{\{2\}, \bullet}(\mathcal{A})$ denote the bi-complex consisting of the columns $\mathrm{CC}^{-1, \bullet}(\mathcal{A})$ and $\mathrm{CC}^{0, \bullet}(\mathcal{A})$ with differentials as in $\mathrm{CC}^{\bullet \bullet \bullet}(\mathcal{A})$.

We recall that we have an exact sequence of complexes

$$
0 \rightarrow \operatorname{Tot}\left(\mathrm{CC}^{\{2\}, \bullet}(\mathcal{A})\right) \stackrel{I}{\rightarrow} \mathrm{Cycl}^{\bullet}(\mathcal{A}) \stackrel{S}{\rightarrow} \mathrm{Cycl}^{\bullet+2}(\mathcal{A}) \rightarrow 0 .
$$

We recall that $\mathrm{CC}^{1, \bullet}(\mathcal{A})$ is acyclic (see p. 411). It follows that $\mathrm{CC}^{\{2\}, \bullet}(\mathcal{A})$ is quasiisomorphic to $C^{\bullet}(\mathcal{A})$. This quasi-isomorphism is realized by the map of complexes taking $a_{k} \in C^{-k}(\mathcal{A})$ to $\left(a_{k}, 0\right) \in C^{-k}(\mathcal{A}) \oplus \operatorname{bar}^{1-k}(\mathcal{A})$. We denote the composite of $I$ with this quasi-isomorphism by $\mathcal{I}$. The map $S$ is obtained by projection to the double complex obtained from $\mathrm{CC}^{\bullet, \bullet}(\mathcal{A})$ by truncating the columns $\mathrm{CC}^{i, \bullet}(\mathcal{A})$ for $i=0,-1$. We now obtain the following proposition.

Proposition 9. If $\mathcal{B}=\operatorname{End}\left(V^{\bullet}\right)$, where $V^{\bullet}$ is a finite dimensional graded $\mathbb{C}$-vector space with zero differential, then the following diagrams commute:
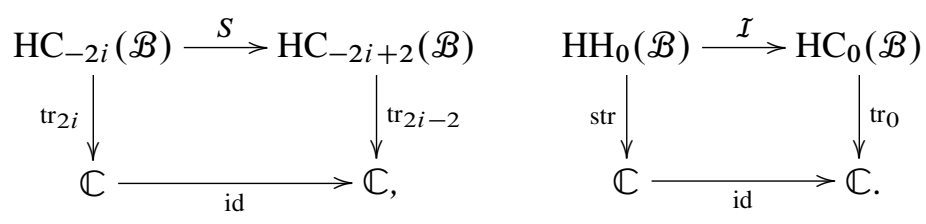

Proof. Let $\left(b_{2 i}, b_{2 i-1}, \ldots, b_{0}\right), b_{j} \in \mathrm{CC}^{-2 i+j,-j}(\mathscr{B})$, be a tuple yielding a cyclic cycle. We already demonstrated while proving part (iii) of Proposition 8 that any class in $\mathrm{HC}_{-2 i}(\mathscr{B})$ can be represented by a cycle coming from a tuple of this form. 
Let $\left.\widehat{\left(b_{2 i}, b_{2 i-1}, \ldots, b_{0}\right)}\right)$ denote the class of $\left(b_{2 i}, b_{2 i-1}, \ldots, b_{0}\right)$ in $\mathrm{HC}_{-2 i}(\mathscr{B})$. Then $\operatorname{tr}_{2 i}\left(\widehat{\left(b_{2 i}, b_{2 i-1}, \ldots, b_{0}\right)}\right)=\operatorname{str}\left(b_{0}\right)$ by Proposition 8 and $S\left(\left(b_{2 i}, b_{2 i-1}, \ldots, b_{0}\right)\right)=$ $\left(b_{2 i-2}, b_{2 i-3}, \ldots, b_{0}\right)$ by the definition of $S$. Finally, by Proposition 8 , $\left.\operatorname{tr}_{2 i-2}\left(\widehat{\left(b_{2 i-2}, b_{2 i-3}, \ldots, b_{0}\right.}\right)\right)=\operatorname{str}\left(b_{0}\right)$. This shows that the first diagram commutes.

Let $b_{0}$ be a Hochschild 0 -cycle of $\mathscr{B}$. Then $\mathcal{I}\left(b_{0}\right)=b_{0} \in \mathrm{CC}^{0,0}(\mathscr{B})$. Let $\overline{b_{0}}$ denote the class of $b_{0}$ in $\mathrm{HH}_{0}(\mathscr{B})$. Then, $\overline{b_{0}}=\operatorname{str}\left(b_{0}\right)$. However, $\mathcal{I}\left(\overline{b_{0}}\right)=\widetilde{b_{0}}$. Now $\operatorname{tr}_{0}\left(\widetilde{b_{0}}\right)=\operatorname{str}\left(b_{0}\right)$ by Proposition 8 . This proves that the second diagram commutes.

Moreover, if $\mathcal{F}: \mathcal{A} \rightarrow \mathscr{B}$ is an $A_{\infty}$-morphism with Taylor components $\mathscr{F}_{k}$, we have the following result.

Proposition 10. The map $\mathcal{F}_{\text {Hoch }}$ mentioned in Proposition 2 extends to a map $\tilde{F}_{\mathrm{Cycl}}$ of complexes from $\mathrm{Cycl}^{\bullet}(\mathcal{A})$ to $\mathrm{Cycl}^{\bullet}(\mathscr{B})$.

Proof. It suffices to check that $\mathcal{F}_{\text {Hoch }}$ extends to a map of $\mathcal{F}_{\text {Tsyg }}$ bi-complexes from $\mathrm{CC}^{\bullet, \bullet}(\mathcal{A})$ to $\mathrm{CC}^{\bullet, \bullet}(\mathscr{B})$.

Let $\mathcal{F}_{\text {Tsyg }}: \mathrm{CC}^{p, q}(\mathcal{A}) \rightarrow \mathrm{CC}^{p, q}(\mathscr{B})$ be $\mathcal{F}_{\text {Hoch }}$ if $p$ is even and non-positive and $\mathcal{F}_{\text {bar }}$ if $p$ is odd and negative.

By Proposition 2, $\widetilde{F}_{\text {Hoch }}: \mathrm{CC}^{p, \bullet}(\mathcal{A}) \rightarrow \mathrm{CC}^{p, \bullet}(\mathscr{B})$ is a map of complexes of $\mathbb{C}$ vector spaces if $p$ is even and non-positive. By the definition of an $A_{\infty}$-morphism, $\widetilde{F}_{\text {bar }}: \mathrm{CC}^{p, \bullet}(\mathcal{A}) \rightarrow \mathrm{CC}^{p, \bullet}(\mathcal{B})$ is a map of complexes if $p$ is odd and negative.

The following verifications, which we leave to the reader, complete the proof that $\mathcal{F}_{\text {Tsyg }}$ is a map of bi-complexes, and thus yields a map $\mathscr{F}_{\mathrm{Cycl}}: \operatorname{Cycl}^{\bullet}(\mathcal{A}) \rightarrow \operatorname{Cycl}^{\bullet}(\mathcal{B})$ of complexes:

$$
\begin{aligned}
(\mathrm{id}-\tau) \circ \mathscr{F}_{\text {bar }} & =\mathscr{F}_{\text {Hoch }} \circ(1-\tau), \\
N \circ \mathscr{F}_{\text {Hoch }} & =\mathscr{F}_{\text {bar }} \circ N .
\end{aligned}
$$

Here $N$ and $\tau$ are as in the definitions of $\mathrm{CC}^{\bullet, \bullet}(\mathcal{A})$ and $\mathrm{CC}^{\bullet, \bullet}(\mathscr{B})$.

We also state the following consequence of Propositions 10 and 8 as a proposition.

Proposition 11. Let $\mathcal{A}$ be a dg $\mathbb{C}$ algebra. Let $\mathcal{B}$ be as in Proposition 9. Suppose that $\mathcal{F}_{\text {is }}$ an $A_{\infty}$-morphism from $\mathcal{A}$ to $\mathcal{B}$. Let $\widetilde{\mathcal{F}_{\mathrm{Cycl}}}$ denote the map from $\mathrm{HC}$. $(\mathcal{A})$ to HC. $(\mathscr{B})$ induced by $\tilde{F}_{\text {Cycl }}$. Then the following holds:

(1) $\left.\operatorname{tr}_{2 i} \circ \widetilde{\mathcal{F}_{\text {Cycl }}}\left(\widehat{\left(a_{2 i}, \ldots, a_{0}, a_{-1}, \ldots, a_{-l}\right.}\right)\right)=\operatorname{str}\left(\widetilde{F}_{\text {Hoch }}\left(a_{0}\right)\right)$.

(2) If $a_{0}$ is a Hochschild 0 - cycle arising out of a degree $k-1$ element of $\mathcal{A}^{\otimes k}$ then

$$
\operatorname{tr}_{2 i} \circ \widetilde{\mathcal{F}_{\text {Cycl }}}\left(\widetilde{\left(a_{2 i}, \ldots, a_{0}, a_{-1}, \ldots, a_{-l}\right)}\right)=\sum_{s=0}^{s=k-1} \operatorname{str}\left(\mathscr{F}_{k}\left(\tau^{s}\left(a_{0}\right)\right)\right),
$$


where $\tau$ is as in Corollary 2.

Proof. By the proof of Proposition 10, we have

$$
\begin{aligned}
& \mathcal{F}_{\text {Tsyg }}\left(a_{2 i}, \ldots, a_{0}, a_{-1}, \ldots, a_{-l}\right) \\
& \quad=\left(\mathcal{F}_{\text {Hoch }}\left(a_{2 i}\right), \mathcal{F}_{\text {bar }}\left(a_{2 i-1}\right), \ldots, \mathcal{F}_{\text {bar }}\left(a_{1}\right), \mathcal{F}_{\text {Hoch }}\left(a_{0}\right), \ldots, \mathcal{F}_{\text {bar } / \text { hoch }}\left(a_{-l}\right)\right),
\end{aligned}
$$

where $\mathcal{F}_{\text {bar } / \text { Hoch }}\left(a_{-l}\right)=\mathcal{F}_{\text {Hoch }}\left(a_{-l}\right)$ if $l$ is even and $\mathcal{F}_{\text {bar } / \text { Hoch }}\left(a_{-l}\right)=\mathcal{F}_{\text {bar }}\left(a_{-l}\right)$ if $l$ is odd. Part (1) now follows immediately from Proposition 8, part (iii), and part (2) is a consequence of part (1) and Corollary 2.

5.2. The complex $\overline{\operatorname{Cycl}^{\bullet}(\mathscr{D i f f}(\mathcal{E}))}$. Let $\underline{\mathbb{C}}^{\infty}$ denote the sheaf of smooth functions on $X$. For each open $U \subset X$, we can consider the completed Hochschild complex of

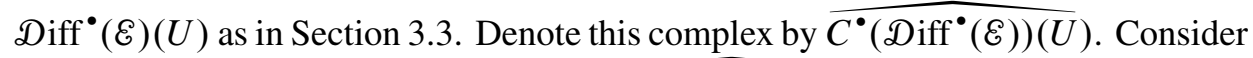

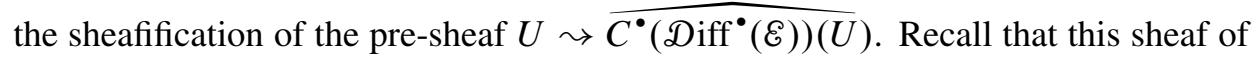
complexes of $\underline{\mathbb{C}}^{\infty}$-modules is denoted by $\widehat{\operatorname{Hoch}(\mathscr{D i f f}(\mathcal{E}))}$ in Section 3.3. Unlike in Section 3.3, let $\widehat{\operatorname{Hoch}^{n}(\mathscr{D i f f}(\mathcal{E}))}$ denote the degree $n$ component of $\widehat{\operatorname{Hoch}(\mathscr{D i f f}(\mathcal{E}))}$.

One can also consider the completed bar complex $\left.\overline{\operatorname{bar}^{\bullet}\left(\operatorname{Diff}^{\bullet}(\mathcal{E})(U)\right.}\right)$. This is defined as in Section 3.3: the underlying graded $\mathbb{C}$-vector space of the complex

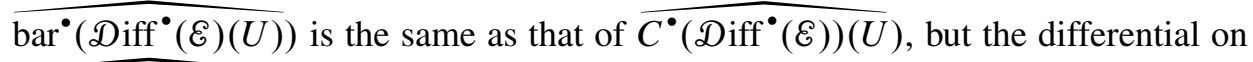
$\operatorname{bar}^{\bullet}\left(\operatorname{Diff}^{\bullet}(\mathscr{E})(U)\right)$ is the bar differential. We will denote the sheafification of the pre-sheaf $\left.U \leadsto \widehat{\operatorname{bar}^{\bullet}\left(\operatorname{Diff}^{\bullet}(\mathcal{E})(U)\right.}\right)$ by $\widehat{\operatorname{bar}^{\bullet}(\mathscr{D i f f}(\mathscr{E}))}$. We have the following result.

Proposition 12. The differentials in Tsygan's double complex for $\operatorname{Diff}^{\bullet}(\mathcal{E})(U)$ extend to yield differentials for the following double complex of $\underline{\mathbb{C}}^{\infty}$-modules on $X$ :

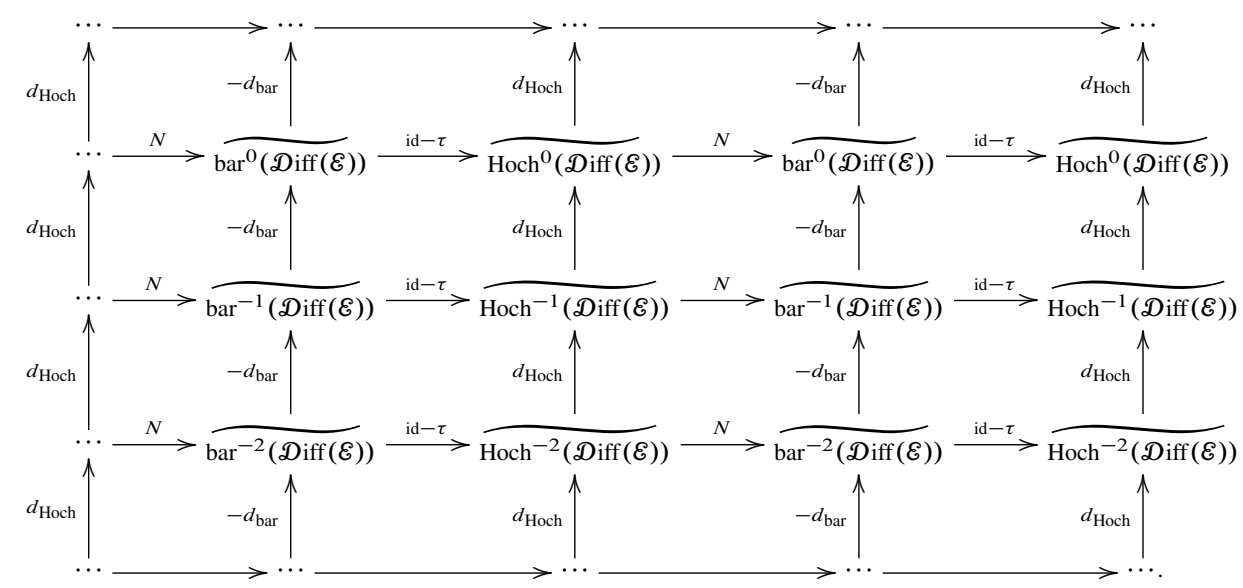


Proof. This only needs to be checked at the level of pre-sheaves. The double complex mentioned is the sheafification of the pre-sheaf $U \leadsto \overline{\mathrm{CC}^{\bullet \bullet}\left(\operatorname{Diff}^{\bullet}(\mathcal{E})(U)\right)}$. Here, $\left.\widehat{\mathrm{CC}^{\bullet} \bullet^{\bullet}\left(\operatorname{Diff}^{\bullet}(\mathscr{E})(U)\right.}\right)$ is the double complex obtained by replacing the Hochschild and bar complexes that make up the columns of Tsygan's double complex of $\operatorname{Diff}^{\bullet}(\mathscr{E})(U)$ with their completed versions. It is also clear that id $-\tau$ and $N$ extend to horizontal differentials on the double complex in this proposition.

We denote the total complex of this double complex by $\widetilde{\mathrm{Cycl}^{\circ}}(\operatorname{Diff}(\mathscr{E}))$. Since it is a complex of $\underline{\mathbb{C}}^{\infty}$ modules, its hypercohomology is computed by the complex $\Gamma\left(X, \widetilde{\operatorname{Cycl}}^{\circ}(\operatorname{Diff}(\mathcal{E}))\right)$.

The following cyclic analog of Proposition 6 follows from Proposition 11 and the fact that $\sum_{s} \tau^{s}(\mathrm{id}-\tau)=0$. We denote by $\widetilde{\mathrm{HC}}_{-2 i}(\mathscr{D i f f}(\mathcal{E}))$ the $-2 i$-th cohomology of $\Gamma\left(X, \widetilde{\operatorname{Cycl}^{\bullet}}(\mathscr{D i f f}(\mathscr{E}))\right)$.

Proposition 13. The formula described in Proposition 11 extends to yield us a $\mathbb{C}$ linear functional $\tilde{\operatorname{tr}}_{2 i}: \widetilde{\mathrm{HC}}_{-2 i}(\operatorname{Diff}(\mathcal{E})) \rightarrow \mathbb{C}$.

Proof. The fact that the formula makes sense follows directly from Proposition 11 and Proposition 6. To show that it vanishes on coboundaries, we recall that Proposition 6 also tells us that it vanishes on the image of the $d_{\text {Hoch }}$ differential. We only need to verify that it vanishes on the image of the id $-\tau$ differential. This is a consequence of the fact that $\sum_{s} \tau^{s}(\mathrm{id}-\tau)=0$.

Recall that we had a map $S: \operatorname{Cycl}^{\bullet}\left(\operatorname{Diff}^{\bullet}(\mathcal{E})\right) \rightarrow \operatorname{Cycl}^{\bullet+2}\left(\operatorname{Diff}^{\bullet}(\mathcal{E})\right)$. It is easy to

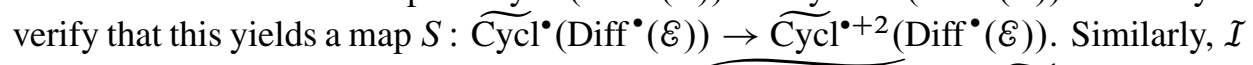

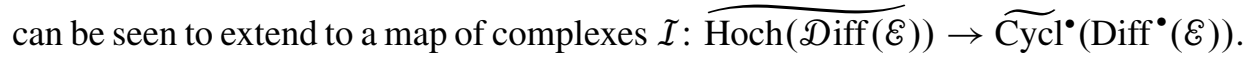
A direct consequence of the formula in Proposition 11 is the following proposition.

Proposition 14. The following diagrams commute:

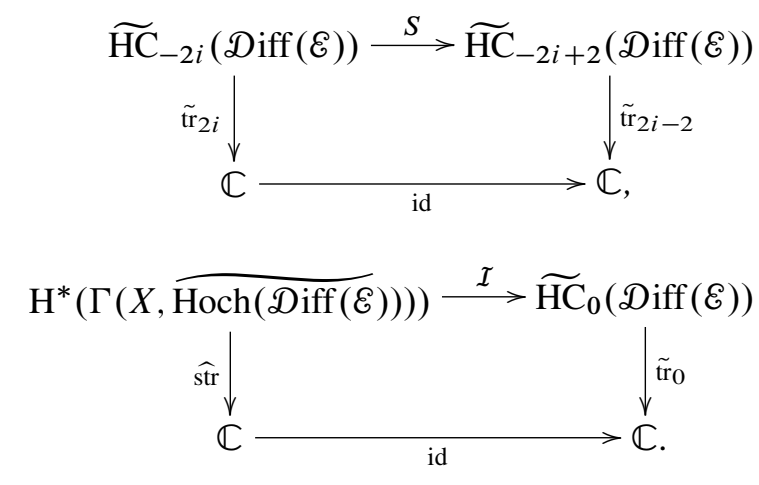




\subsection{The completed cyclic homology of $\mathscr{D i f f}(\mathscr{E})$ and the cohomology of $X$}

5.3.1. The completed cyclic homology of $\mathscr{D} \operatorname{iff}(\mathscr{E})$. We define the completed bar complex of $\operatorname{Diff}(\mathcal{E})$ to be the sheafification of the pre-sheaf $\left.U \rightarrow \overline{\operatorname{bar}^{\bullet}(\operatorname{Diff}(\mathcal{E})(U)}\right)$. The terms of this complex are the same as in $\widehat{\operatorname{Hoch}}(\operatorname{Diff}(\mathcal{E}))$, but the differential is the bar differential. We denote this by $\widehat{\operatorname{bar}}(\mathscr{D i f f}(\mathcal{E}))$.

We can obtain the completed Tsygan double complex of $\mathscr{D i f f}(\mathscr{E})$. This is a double complex of sheaves of $\mathbb{C}$-vector spaces on $X$, whose non-positive even columns are $\widehat{\operatorname{Hoch}}^{\bullet}(\operatorname{Diff}(\mathcal{E}))$ and whose negative odd columns are $\widehat{\operatorname{bar}}(\mathscr{D i f f}(\mathcal{E}))$. The horizontal differentials in Tsygan's double complex extend to this situation to give us well-defined horizontal differentials. We will denote the total complex of this double complex by $\widehat{\operatorname{Cycl}}^{\circ}(\operatorname{Diff}(\varepsilon))$.

Before we proceed, we note that as before we have a (degree preserving) natural map of complexes $\gamma: \widehat{\operatorname{Cycl}}^{\bullet}(\operatorname{Diff}(\mathcal{E})) \rightarrow \widehat{\operatorname{Cycl}}^{\bullet}(\mathscr{D i f f}(\mathcal{E}))$. This induces a map $\gamma_{*}: \mathbb{H}^{\bullet}\left(X, \widehat{\mathrm{Cycl}}^{\bullet}(\operatorname{Diff}(\mathcal{E}))\right) \rightarrow \mathbb{H}^{\bullet}\left(X, \widetilde{\operatorname{Cycl}^{\bullet}}(\mathscr{D} \operatorname{iff}(\mathcal{E}))\right)$. Note that the map $S$ extends to a map of complexes $\widehat{\mathrm{Cycl}^{\bullet}}(\mathscr{D i f f}(\mathscr{E})) \rightarrow \widehat{\mathrm{Cycl}}^{\bullet+2}(\mathscr{D i f f}(\mathscr{E}))$. Similarly, the map $\mathcal{I}$ extends to a map of complexes $\mathcal{I}: \widehat{\operatorname{Hoch}}(\operatorname{Diff}(\mathscr{E})) \rightarrow \widehat{\mathrm{Cycl}^{\circ}}(\mathscr{D i f f}(\mathcal{E}))$. Furthermore, we have the following commutative diagrams:

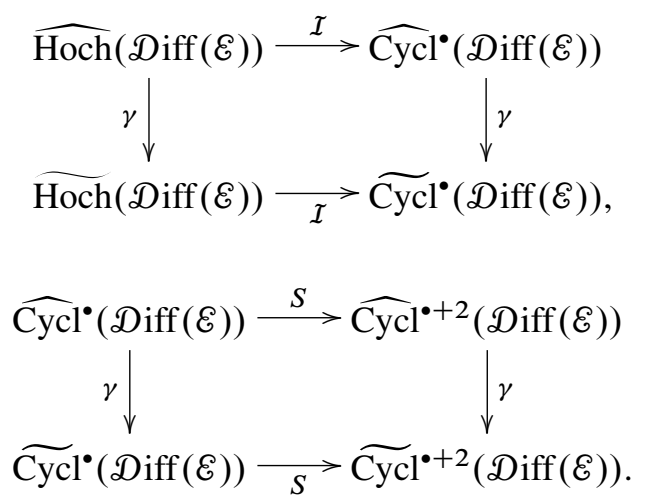

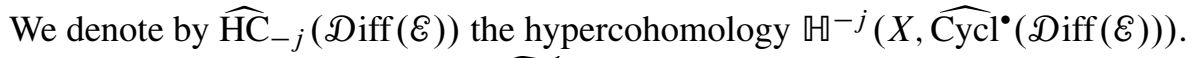
Again we note that the complex $\widetilde{\mathrm{Cycl}^{\circ}}(\operatorname{Diff}(\mathscr{E}))$ is a complex of $\mathbb{\mathbb { C }}^{\infty}$ modules. Thus, its hypercohomology is precisely $\widetilde{\mathrm{HC}}$. $(\mathscr{D} \operatorname{iff}(\mathcal{E}))$. It follows that we have the following corollaries of Propositions 13 and 14, respectively.

Corollary 7 (Corollary to Proposition 13). There exist traces

$$
\widehat{\operatorname{tr}}_{2 i}: \widehat{\mathrm{HC}}_{-2 i}(\operatorname{Diff}(\mathcal{E})) \rightarrow \mathbb{C} \text {. }
$$

Proof. This follows from $\hat{\operatorname{tr}}_{2 i}=\tilde{\operatorname{tr}}_{2 i} \circ \gamma_{*}$. 
Corollary 8 (Corollary to Proposition 14). The following diagrams commute:
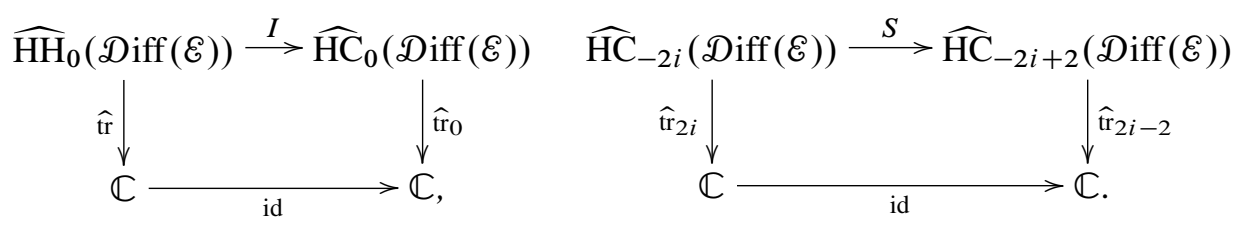

Proof. This is an immediate consequence of Proposition 14 and the two commutative diagrams shown at the top.

We have the following cyclic homology analog of Lemma 3.

Lemma 5. $\widehat{\operatorname{Cycl}}^{\bullet}(\operatorname{Diff}(\mathcal{E}))$ is quasi-isomorphic to $\underline{\mathbb{C}}[2 n] \oplus \underline{\mathbb{C}}[2 n+2] \oplus \cdots$.

Proof. It suffices to verify this assertion locally at the level of pre-sheaves. In other words, we need to look at the completed Tsygans double complex for $\operatorname{Diff}(\mathcal{E})(U)$ where $U$ is an open set so that $\varepsilon$ is trivial on $U$. Of course, all tensor products here are completed tensor products.

The completed bar complex of $\operatorname{Diff}(\mathscr{E})(U)$ is acyclic since the homotopy of recollection 1, Section 2.1 between the identity endomorphism and the 0 endomorphism of the bar complex of $\mathscr{D i f f}(\mathscr{E})(U)$ can be shown to "extend to" a homotopy between the identity and 0 endomorphisms of the completed bar complex of $\operatorname{Diff}(\mathscr{E})(U)$. The complex $\overline{\operatorname{Hoch}(\mathscr{D i f f}(\mathscr{E})(U)})$ is quasi-isomorphic to $\mathbb{C}[2 n]$ by Lemma 3 . It follows that the spectral sequence converging to the completed cyclic homology of $\operatorname{Diff}(\mathscr{E})(U)$ that arises out of the filtration of the completed Tsygan double complex for $\operatorname{Diff}(\mathscr{E})(U)$ by columns satisfies:

$$
E_{1}^{-2 i,-2 n} \simeq \mathbb{C} \text { for all } i \geq 0 \text { and } E_{1}^{p, q}=0 \text { otherwise. }
$$

The lemma now follows from this.

Corollary 9. $\widehat{\mathrm{HC}}_{-2 i}(\mathscr{D i f f}(\mathcal{E}))=\mathrm{H}^{2 n}(X, \mathbb{C}) \oplus \mathrm{H}^{2 n-2}(X, \mathbb{C}) \oplus \cdots \oplus \mathrm{H}^{2 n-2 i}(X, \mathbb{C})$.

Denote the isomorphism in the above corollary by $J_{2 i}$. Then, following the proof of Lemma 5, we obtain the following result.

Proposition 15. The following diagrams commute:

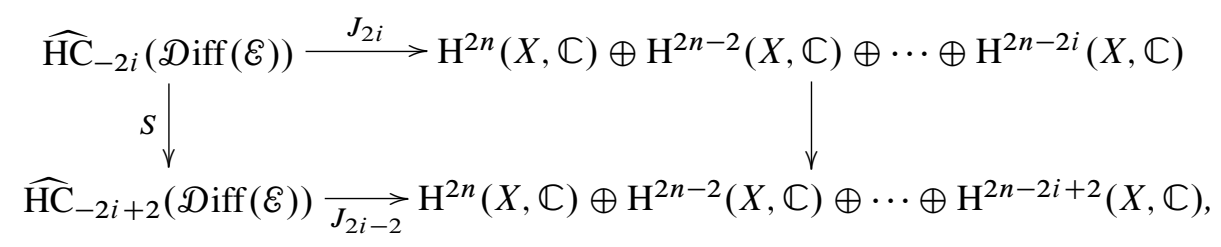




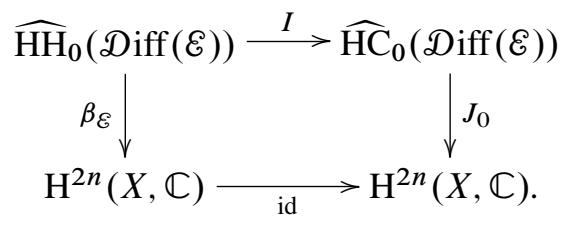

The vertical arrow in the right column of the first diagram is the obvious projection.

\subsection{Proof of Theorem 3}

Proof of Theorem 3. We apply the second diagram in Corollary $8 i$ times to obtain the following diagram:

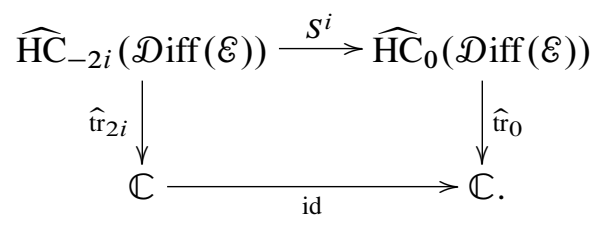

We apply the first diagram Proposition $15 i$ times to obtain the following diagram:

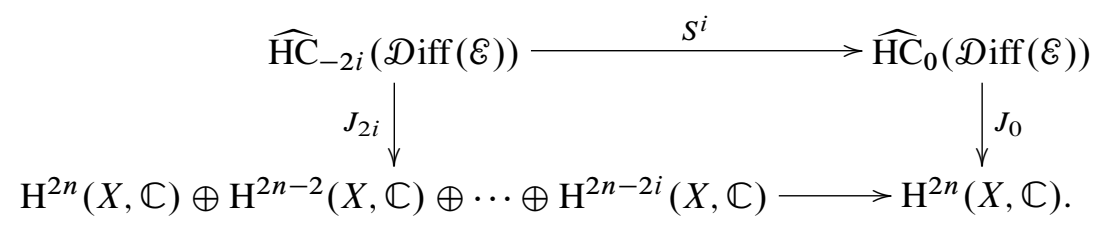

In the notation used to state Theorem 3, this tells us that $I_{\mathcal{E}, 2 i, 0}=I_{\mathcal{E}, 0,0}$ and $I_{\mathcal{E}, 2 i, 2 k}=0$ for $k>0$. Lastly, the second diagram of Proposition 15 and the first diagram of Corollary 8 together imply that $\widehat{\operatorname{tr}} \circ \beta_{\mathcal{E}}^{-1}=\widehat{\mathrm{r}_{0}} \circ J_{0}^{-1}$. This shows that $I_{\mathcal{E}, 0,0}=I_{\mathcal{E}}$, which completes the proof.

Remark 1. Our notion of completed Hochschild and cyclic homologies seems to differ in detail from that used in [FLS]. This forced us to rework some steps of [FLS] - in particular, Proposition 6 and related matters - in our situation.

Remark 2. The key step in the proof of Theorem 1 in [FLS] consists in showing, on the one hand, that the linear functional defined in [FLS] applied to the operator id in $\operatorname{Diff}(\mathcal{E})$ is indeed its supertrace, i.e., the Euler characteristic of $\mathcal{E}$, and showing that the image of id in $\widehat{\mathrm{HH}}_{0}\left(\operatorname{Diff}(\mathcal{E})\right.$ gives the class $\mathrm{Ch}(\mathcal{E}) \cdot \operatorname{Td}\left(T_{X}\right)_{2 n}$ (this is done by citing [NT1] and [NT2]) after passing to $\mathrm{H}^{2 n}(X, \mathbb{C})$ and then applying the Hirzebruch $\mathrm{R}-\mathrm{R}$ theorem. In spite of the differences in detail between our construction of completed Hochschild homology and that of [FLS] it may be checked that these two key steps go through, thus maintaining Theorem 1 in this situation. 


\section{References}

[BGV] N. Berline, E. Getzler, and M. Vergne, Heat kernels and Dirac operators. Grundlehren Text Editions, Springer-Verlag, Berlin 2004. Zbl 1037.58015 MR 2273508

[Bryl] J.-L. Brylinski, A differential complex for Poisson manifolds. J. Differential Geom. 28 (1988), 93-114. Zbl 0634.58029 MR 950556

[EnFe] M. Engeli and G. Felder, A Riemann-Roch-Hirzebruch formula for traces of differential operators. Preprint 2007. arXiv:math.QA/0702461

[FLS] B. Feigin, A. Losev, and B. Shoikhet, Riemann-Roch-Hirzebruch theorem and topological quantum mechanics. Preprint 2004. arXiv:math.QA/0401400

[Groth] A. Grothendieck, Produits tensoriels topologiques et espaces nucléaires. Mem. Amer. Math. Soc. 16 (1955). Zbl 0064.35501 MR 0075539

[Lod] J.-L. Loday, Cyclic homology. 2nd ed., Grundlehren Math. Wiss. 301, Springer-Verlag, Berlin 1998. Zbl 0885.18007 MR 1600246

[NT1] R. Nest and B. Tsygan, Algebraic index theorem. Comm. Math. Phys. 172 (1995), 223-262. Zbl 0887.58050 MR 1350407

[NT2] R. Nest and B. Tsygan, On the cohomology ring of an algebra. In Advances in geometry, Progr. Math. 172, Birkhäuser, Boston 1999, 337-370. Zbl 0919.57019 MR 1667686

[Riet] K. Rietsch, An introduction to perverse sheaves. In Representations of finite dimensional algebras and related topics in Lie theory and geometry, Fields Inst. Commun. 40, Amer. Math. Soc., Providence, RI, 2004, 391-429. Zbl 1058.55002 MR 2057405

[S-S] P. Schapira and J.-P. Schneiders, Index theorem for elliptic pairs. Astérisque 224 (1994). Zbl 0856.58037 MR 1305641

[Tel] N. Teleman, Microlocalisation de l'homologie de Hochschild. C. R. Acad. Sci. Paris Sér. I Math. 326 (1998), 1261-1264. Zbl 0931.58015 MR 1649133

[Vois] C. Voisin, Hodge theory and complex algebraic geometry I. Cambridge Stud. Adv. Math. 76, Cambridge University Press, Cambridge 2002. Zbl 1005.14002 MR 1967689

Received September 17, 2007; revised March 11, 2008

A. C. Ramadoss, Department of Mathematics, University of Oklahoma, Norman,

OK 73019-0315, U.S.A.

E-mail: aramadoss@math.ou.edu 